\title{
Molecular Diversity Among Adult Human Hippocampal and
}

\section{Entorhinal Cells}

Daniel Franjic ${ }^{1}$, Jinmyung Choi ${ }^{1}$, Mario Skarica ${ }^{1}$, Chuan Xu' ${ }^{1}$, Qian Li ${ }^{1}$, Shaojie Ma ${ }^{1}$,

Jankovic-Rapan', Andre M. M. Sousa1, Pasko Rakic'1,4, Nenad Sestan ${ }^{1,3,4, *}$

${ }^{1}$ Department of Neuroscience, Yale School of Medicine, New Haven, CT, USA.

${ }^{2}$ Neurogenomics Group, Research Programme on Biomedical Informatics, Hospital del Mar

11 Medical Research Institute, Department of Experimental and Health Sciences, Universitat

12 Pompeu Fabra, Barcelona, Catalonia, Spain

$13{ }^{3}$ Departments of Genetics, Psychiatry, and Comparative Medicine, Program in Cellular

14 Neuroscience, Neurodegeneration and Repair, and Yale Child Study Center, Yale School of

15 Medicine, New Haven, CT, USA.

${ }^{4}$ Kavli Institute for Neuroscience, Yale School of Medicine, New Haven, CT, USA. 


\section{SUMMARY}

25 The hippocampal-entorhinal system is comprised of functionally distinct subregions

26 collectively critical for cognition, and selectively vulnerable to aging and pathological

27 processes. To gain insights into neuronal and non-neuronal populations within this system, we performed single-nucleus transcriptional profiling from five human hippocampal-entorhinal

29 subregions. We found that transcriptomic diversity of excitatory neurons across these

30 subregions reflected the molecular transition from three-layered archicortex to six-layered

31 neocortex. Additionally, mRNA and protein for DCX, an immature neuron marker, were clearly

32 detected in some cells, but not in dentate granule cells, the cell-type predicted to be generated

33 in adult neurogenesis. We also found that previously functionally uncharacterized METTL7B

34 was enriched in human and non-human primate neuronal subtypes less vulnerable to initial

35 Alzheimer's disease pathology. Proteomic and biochemical assays revealed METTL7B interacts

36 with Alzheimer's disease-related proteins, including APP, and its overexpression reduced

37 amyloid-beta generation. These results reveal cell type-specific molecular properties relevant

38 for hippocampal-entorhinal physiology and dysfunction. 


\section{INTRODUCTION}

The neural circuits of the hippocampal formation (HIP) and entorhinal cortex (EC) are critical components of a widespread neural network for memory and representation of space and time (Gloor, 1997; Andersen, 2007; Buzsaki and Moser, 2013). Based on cytoarchitectonic, cellular, and circuitry variations, the hippocampal-entorhinal system can be subdivided into functionally distinct subregions that gradually transition from the simple three-layered dentate gyrus (DG) and hippocampus (Cornu Ammonis, CA), and through more complex lamination of the subiculum (collectively referred to as the allocortex) to the six-layered EC (mesocortex) (Freund, 2002; Suzuki and Amaral, 2004; Klausberger and Somogyi, 2008). The molecular basis of the diversity of cell types in these subregions and their homology with bordering neocortical cell types and lamination remains poorly understood (Kriegstein and Connors, 1986; Hoogland and Vermeulen-Vanderzee, 1989; Reiner, 1991; Ishizuka, 2001; Zeisel et al., 2015; Cembrowski et al., 2016b; Mercer and Thomson, 2017; Shepherd and Rowe, 2017).

Primate- or human-specific evolutionary innovations may underlie some regionselective aspects of hippocampal cell types, necessitating the study specifically of the human hippocampus. The cytoarchitectonic differentiation of the allocortex and neocortex arose very early during mammalian evolution, with the mammalian allocortex reminiscent of the threelayered reptilian cortex rather than six-layered mammalian cortex. Based on this similarity, as well as histological, physiological and connectional studies, it is hypothesized that allocortex is composed of excitatory projection neurons that resemble those specifically in the deep layers of the mammalian neocortex (Kriegstein and Connors, 1986; Reiner, 1991; Ishizuka, 2001; Luzzati, 2015; Shepherd and Rowe, 2017). However, although the hippocampus is a phylogenetically ancient part of the cerebral cortex, the hippocampal-entorhinal system and its 
cortico-cortical projections are greatly expanded in humans and non-human primates (Stephan, 1975; Demeter et al., 1990; Morrison and Hof, 1997; Suzuki and Amaral, 2004; Patzke et al., 2015). Moreover, the human and non-human primate homologs of this system have undergone extensive evolutionary changes also in gene expression and perhaps cell composition as compared to some commonly studied mammals such as rodents (Bakken et al., 2015; Sousa et al., 2017). As a potential consequence, the normal functions and disease features of cell types in these subregions may be absent or exhibit substantial differences in other species. For example, a distinctive feature of the hippocampal-entorhinal systems of most analyzed mammals (apart from cetaceans) is persistent adult neurogenesis of excitatory granule neurons in the DG (Patzke et al., 2015). However, whether new granule cells are generated in the adult human DG, and whether these neurons express DCX, a marker for immature neurons that is associated with neurogenesis, has not been fully resolved (Eriksson et al., 1998; Rakic, 2002; Spalding et al., 2013; Boldrini et al., 2018; Kempermann et al., 2018; Sorrells et al., 2018; Moreno-Jimenez et al., 2019).

Within the human hippocampal-entorhinal system, some cell types and circuits are selectively vulnerable in normal aging and certain pathological processes. For example, excitatory projection (pyramidal) neurons in the hippocampal CA1 field (Sommer's sector), and to a lesser degree those in CA2-4 fields, are more vulnerable to hypoxia-ischemia damage and intractable seizures (mesial temporal sclerosis) than DG granule cells or other major hippocampal neuronal subtypes (Schmidt-Kastner and Freund, 1991; Blumcke et al., 2007). Alzheimer disease's pathology, which is defined by extracellular accumulation of A $\beta$ peptides and intracellular aggregates of hyperphosphorylated tau protein, also exhibits regional and cellular differences (Fischer, 1907; Glenner and Wong, 1984; Alzheimer et al., 1995; Hardy 
and Selkoe, 2002; Tanzi and Bertram, 2005; Ballatore et al., 2007; Karran and De Strooper, 2016). In mesial temporal regions, pathology first appears in layer 2 of the EC and hippocampal CA1 field (McMenemey, 1940; Wilcock and Esiri, 1982; Morrison and Hof, 1997; SerranoPozo et al., 2011; Braak and Del Trecidi, 2015), while DG cells, CA2-4 pyramidal neurons and layer 5B pyramidal neurons in the EC (Davies et al., 1992; Jin et al., 2004; Schonheit et al., 2004; West et al., 2004; Ohm, 2007) are more resilient in the early stages of the disease. Given this selectivity, a more detailed molecular profiling of this system, will aid our understanding of human brain development and neuropsychiatric disease.

To gain new insights into cell populations and cell type-specific differences in gene expression, evolution, neurogenic capability, and variable disease susceptibility, we performed high-coverage single-nucleus RNA sequencing (snRNA-seq) on five anatomically defined subregions of the hippocampal-entorhinal system. These efforts, like similar recent efforts to transcriptomically characterize the postmortem adult human brain (Krishnaswami et al., 2016; Lake et al., 2016; Habib et al., 2017; Lake et al., 2018; Li et al., 2018; Hodge et al., 2019; Mathys et al., 2019; Schirmer et al., 2019; Velmeshev et al., 2019) (including pioneering profiling of HIP (Habib et al., 2017), identified a highly diverse set of neuronal and nonneuronal cell types with clear regional distinctions and implications for human brain function, evolution, and disease.

\section{RESULTS}

\section{Transcriptomic diversity of hippocampal and entorhinal cells}

To survey the transcriptomic diversity and functional specification of the mesial temporal cortex, we used snRNA-seq to profile five subregions of the hippocampal-entorhinal system 
108

109

110

111

112

113

114

115

116

117

118

119

120

121

122

123

124

125

126

127

128

129

130

collected from fresh frozen postmortem brains of clinically unremarkable human donors. These specimens were selected from a larger pool of postmortem human brains based on the quality of isolated nuclei and RNA. Taking into consideration dramatic cytoarchitectonic variations, we microdissected the hippocampal formation (DG, CA2-4, CA1, and subiculum) and EC for a total of five subregions (Fig. 1A).

Unbiased isolation of nuclei using our previously described protocol (Li et al., 2018; Zhu et al., 2018) followed by snRNA barcoding, cDNA sequencing and quality filtering yielded 108,315 high-quality single-nucleus profiles from all five subregions (Fig. 1A, S1A-D). Analysis of the expression of genes known to be enriched in major cell subpopulations suggested these included 44,697 neurons, of which 35,768 (80.02\%) were glutamatergic excitatory neurons (expressing the gene encoding the vesicular glutamate transporter $S L C 17 A 7$ ) and $8,929(19.98 \%)$ were GABAergic inhibitory neurons (expressing the gene encoding the GABA synthesis enzyme $G A D 1$ ), reflecting the expected 80:20 ratio of these populations. In addition, we identified 63,618 (58.73\% of the total population) non-neuronal cells.

We next analyzed the transcriptomes of those nuclei on the Uniform Manifold Approximation and Projection (UMAP) layout representing their similarities at cellular granularity (Fig. 1B-D). Iterative clustering defined 69 transcriptomically distinct cell clusters representing presumptive cell types across all individuals (donors). These transcriptomically diverse subpopulations were organized into a dendrogramatic taxonomy reflecting their gene expression patterns and were subsequently assigned identities commensurate with predicted cell types. This allowed us to identify 26 subtypes of excitatory neurons (Fig. 1E and S2A-B), 23 inhibitory neuron subtypes (Fig. 1E and S2C-D), and 20 non-neuronal cell types and subtypes

(Fig. 1E and S2E-F). Similar single nucleus approaches applied to human neocortical samples 
131 yielded comparable numbers and distributions of cell populations in medial temporal gyrus 132 (MTG) (Hodge et al., 2019)and dorso-lateral prefrontal cortex (dlPFC) (Li et al., 2018)(Fig. S1E-F).

Within excitatory neuron subtypes, we found marked transcriptional diversity that reflects differences in the cytoarchitectonic organization among the subregions of the HIP (DG, CA2-4, CA1, and subiculum) and EC (Fig. 1E). For example, in addition to ADCYAP1expressing mossy cells in DG, we found three distinct subclusters of $P R O X 1$-expressing granule cells. We also identified excitatory neurons in CA1 and CA2-4 that could be deconstructed into additional subtypes, indicating a finer molecular subdivision not readily apparent in the cytoarchitecture. Molecular distinctions were also evident in the subiculum, with two proximal subtypes close to $\mathrm{CA} 1$ expressing $R O B O 1$ and a third, distal subtype expressing FN1 (Cembrowski et al., 2018). Within the EC, excitatory neurons were broadly characterized by laminar positioning. We identified seven neuron subtypes in layer $2 / 3$ (characterized by high expression of $C U X 2$ and/or $R E L N$ ) and eight subtypes in deep layers 5 and six with specific expression of deep-layer markers including TLE4, ADRA1A, and THEMIS.

In contrast to excitatory neurons, interneurons and non-neuronal cell types exhibited a more uniform spatial distribution (Fig. 1E). Transcriptomic diversity and multiple cell subtypes were evident among these populations, but this diversity did not generally segregate by percent region (middle histogram). For example, although the abundance of some interneuron subtypes differed between HIP and EC, all major subtypes of interneurons, including SST-, PVALB-, $V I P$-, and $L A M P 5$-expressing interneurons, were shared across all subregions assayed. Among multiple non-neuronal cell types, we identified two astrocyte subtypes (Astro), two subtypes of oligodendrocyte precursor cells (OPCs), four subtypes of oligodendrocytes (Oligo), two 
154 subtypes of microglia (Micro), and multiple vasculature subtypes, each of which was generally

155 shared across all of the five subregions dissected. These data therefore describe previously

156 uncharacterized cell populations in the hippocampal- entorhinal system and extend previous

157 findings concerning the functional specificity of neuronal and non-neuronal populations to this system (Freund, 2002; Suzuki and Amaral, 2004; Klausberger and Somogyi, 2008).

159

\section{Taxonomic relationships across allo-, meso- and neo-cortex}

The putative homology between neurons in the hippocampal-entorhinal system and neocortical neurons, and in particular the cytoarchitectonic and evolutionary transition between allo-, meso-, and neo-cortex, offers an opportunity to reveal organizational principles underlying the specialization and function of the mammalian cerebral cortex. Towards elucidating these principles, we compared cell profiles across hippocampal-entorhinal subregions and transcriptomically defined cell types within two human neocortical regions (MTG and dlPFC).

Among hippocampal-entorhinal subregions, we observed a clear distinction between excitatory neurons of the hippocampus proper and DG, and the hippocampal formation more generally, as compared to those of EC (Fig. 2A-B, S2A-B). Excitatory neurons of the CA fields, subiculum and DG were also clearly distinct from those of MTG and dlPFC (Fig. 2B). We did, however, observe transcriptomic similarities between excitatory neurons in all neocortical layers in MTG and dlPFC (Fig. 3A). In contrast, we did not identify excitatory neurons in DG, CA fields, subiculum, and EC that corresponded to all neocortical layers in MTG and dIPFC.

In particular, we identified three major transcriptomically-defined subtypes of excitatory neurons within subiculum (S), two within CA1, and only one predicted within CA2 and CA3, which is consistent with previous single cell RNA-seq studies in rodents (Zeisel et al., 2015; 
177 Cembrowski et al., 2016b) and evidence of the laminar organization of pyramidal (excitatory neurons) within the CA fields (Nielsen et al., 2010; Slomianka et al., 2011; Cipriani et al., 2016). Moreover, we found that deep-layer excitatory neuron subtypes in the neocortex were wellrepresented in the EC and to a lesser extent in the HIP, but upper-layer neuron subtypes were not well represented (Fig 3A-B and S3A). For example, we identified two $R E L N$-expressing layer 2 subclusters in the EC that, similar to a previous report (Witter et al., 2017), did not correspond closely to any excitatory neuron subtype detected in the neocortex. Consistent with this observation, molecular markers for deep-layer excitatory neurons in the neocortex displayed higher expression in each subtype of HIP as compared to upper-layer molecular markers (Fig. S3B). Moreover, we observed lower expression of key molecular markers of intratelencephalic

187 (intracerebral) projection neurons in each of the HIP excitatory neuron subtypes as compared to other neocortical neuron populations. Fig. S3C), which may be relevant to the previous observation that HIP in rodents CA fields don't have callosally projecting intratelencephalic/intraceberal excitrory neurons (Cenquizca and Swanson, 2007). Several key genes expressed by excitatory neurons, including FNDC1, RTP5, and PTGFR, also exhibited specificity for allo-, meso-, or neo-cortex. transition between allo-, meso-, and neo-cortex similar to that observed for excitatory neurons, with just one SST-expressing interneuron population in the hippocampus (InN SST ADAMTS12)

196 lacking a clear counterpart in EC, MTG, and dlPFC (Fig. 2D and 3C). Cells in this 197 hippocampal-specific interneuron population were notable for their expression of two EvC 198 Ciliary Complex genes, EVC and EVC2 (Caparros-Martin et al., 2013) (Fig. 3D), which may 199 play a role in hippocampal ciliary sonic hedgehog signaling (Breunig et al., 2008; Rhee et al., 
2016; Park et al., 2019). Lastly, non-neuronal cell types constituted the most transcriptomically conservative populations across the allo-, meso-, and neo-cortical taxonomy, with a high similarity observed in each subtype across all regions (Fig. 2E-F and S2E-J). Taken together, these finding indicate that most prominent differences across allo-, meso-, and neo-cortex occur among excitatory neurons, including the increased prevalence of intratelencephalic projection neurons in the neocortex as compared to allocortex.

\section{Transcriptomic insights into adult hippocampal neurogenic capacity}

Neurogenesis of granule cells in the adult DG has been extensively studied in rodents and documented in many mammalian species. Many of these studies investigate the presence of cells expressing DCX, a marker of immature neurons, as a reliable indicator of newly generated neurons in DG (Couillard-Despres et al., 2005; Patzke et al., 2015; Kempermann et al., 2018). However, there is no consensus regarding the existence of significant neurogenesis in the adult human DG. Previous studies have provided evidence for the generation of granule cells in the adult and aged human DG through the detection of cell proliferation (Eriksson et al., 1998;

Spalding et al., 2013), and a recent study reported a prominent population of DCX-expressing cells in the adult human DG (Moreno-Jimenez et al., 2019). Consistent with these observations, $D C X$ gene expression is detected in the adult and aged human HIP, albeit dramatically lower than in the developing human or adult macaque HIP (Sousa et al., 2017; Zhu et al., 2018). Conversely, other studies have directly challenged this conclusion, having failed to identify neural progenitors or DCX-expressing granule cells after childhood in the adult human DG (Dennis et al., 2016; Cipriani et al., 2018; Sorrells et al., 2018). To add insight to these controversial sets of observations, we investigated our snRNA-seq data set to identify cells that 
may express $D C X$ and other key gene markers related to proliferation and early neuronal differentiation that were previously characterized in adult DG neurogenesis.

Although we observed moderate expression of $D C X$ in some excitatory neurons, and generally greater expression in many interneuron subtypes across the hippocampal-entorhinal system (Fig. 4A-B, S4A), we did not identify discrete clusters of $D C X$-expressing cells in HIP. However, we found 125 cells within the cluster of 14,703 DG granule cells with at least one $D C X$ mRNA molecule (UMI $\geq 1$ ) (Fig. 4C). To further characterize these $D C X$-expressing cells, we assessed whether they were enriched for markers indicative of intermediate progenitor cells and immature granule cells, the cell types previously shown to express $D c x$ during adult DG neurogenesis (Couillard-Despres et al., 2005; Patzke et al., 2015; Kempermann et al., 2018). Although some of these cells co-expressed migrating or immature neuron markers including NEUROD2 (61.6\%), FXYD7 (32.8\%), and NCAM1 (97.6\%) (UMI $\geq 1$ ) (Hochgerner et al., 2018) (Fig. 4E), we found that these percentages were generally comparable or lower than those from excitatory neurons of EC $(51.9 \%, 61.9 \%$, and 99\% for NEUROD2, FXYD7, and NCAM1, respectively) (Fig. 4D, F), a region where adult newborn neurons have not been reported to be generated. Moreover, we did not observe enrichment for these markers as compared to $D C X$ non-expressing DG granule cells (nominal p-value > 0.05) (Fig. S4A). Similarly, putative neural progenitor cells expressing MKI67, which precede immature neurons in development, did not constitute an independent cluster in DG (Fig. S4B). We also found no evidence that neural stem cells clustered with astrocytes, a transcriptomically similar cell population, as all $N E S$-expressing cells in the astrocytic cluster co-expressed AQP4 (61 of 61 cells), a marker for differentiated cells committed to the astrocytic lineage (Fig. S4C). Far fewer of these cells 
expressed $H O P X$ ( 8 of 61 cells), a reported marker for quiescent progenitors responsible for adult neurogenesis in mouse (Berg et al., 2019).

We complemented these snRNA-seq analyses using immunohistochemistry with two different commonly used antibodies against DCX. As recently reported (Sorrells et al., 2019), we detected many DCX-immunopositive neurons in the paralaminar nuclei of the adult human amygdala (Fig. 4G). In contrast, although we detected $D C X$ transcripts in all brains processed for snRNA-seq, immunohistochemical detection of DCX in the hippocampal-entorhinal system was successful in less than one-third of an independent cohort of postmortem adult human samples $(\mathrm{n}=11$; Table S1). This included scarce DCX-immunopositive neurons in the subiculum that were weakly immunopositive for the inhibitory neuron marker GAD1, and the EC, which were not GAD1-immunopositive (Fig. 4G). Moreover, we were unable to detect DCX-immunopositive cells in the DG or the adjacent CA4 field, including in one brain also used for snRNA-seq (total of 12 brains), indicating a poor correlation between detection of DCX mRNA and protein in the postmortem adult human samples. Together, these findings are consistent with recent DCX immunohistochemical studies (Dennis et al., 2016; Cipriani et al., 2018; Sorrells et al., 2018) showing that neurogenesis does not continue, or is extremely rare, in the adult human DG.

\section{Species, age and excitatory neuron subtype-specific $M E T T L 7 B$ expression}

Tissue and single cell expression profiles, including from multiple subregions of the HIP and EC, allowed us to integrate regional and cell type-specific differences in disease susceptibility with temporal patterns of gene expression. We began by identifying candidate genes enriched in the hippocampus and whose expression changes with age as described in a developmental and multi- 
regional human brain transcriptome dataset we previously generated (Kang et al., 2011; Li et al., 2018). Genes were ranked based on their region-specific changes in expression over development and aging (Fig. 5A and Table S2). Within the hippocampus, of the three genes exhibiting the greatest increased (KL, METTL7B, PTGS1) or decreased expression (TSHR, MSTN, WNT8B) across time, five have been previously functionally characterized to some extent, and the two that exhibited a progressive increase ( $K L$ and $P T G S 1)$ have been associated with Alzheimer's disease (Qin et al., 2003; Zeldich et al., 2014).. In contrast, the second-ranked upregulated gene, METTL7B, has not been comprehensively studied in the context of the vertebrate brain. METTL7B, which is predominantly expressed in liver (Uhlen et al., 2015), encodes a membrane protein associated with endoplasmic reticulum (ER) and lipid droplets, and, by amino acid sequence homology, is predicted to belong to the protein methyltransferase superfamily (Turro et al., 2006; Thomas et al., 2013). We confirmed that at the RNA and protein level, METTL7B is enriched in the adult human hippocampus (Fig. 5B-D). Analysis of METTL7B in the same 16 homologous brain regions in chimpanzee and rhesus macaque (Sousa et al., 2017; Zhu et al., 2018) found that expression in the hippocampus, and the cerebrum more generally, is not distinct to humans. However, METTL7B was more broadly expressed throughout the cerebrum in rhesus macaque brain (Fig. S5A). The expression of METTL7B was also enriched in the human and macaque cerebrum as compared to the cerebrum of mouse, rat, rabbit, and opossum (Cardoso-Moreira et al., 2019) (Fig. S5B).

We next mapped the cell type expression of $M E T T L 7 B$, and found it is virtually exclusive to excitatory neurons, with highest enrichment in the DG, followed by CA2-3 and then subiculum (Fig. 5E). RNA in situ hybridization and immunolabeling of adjacent sections confirmed that the highest signal intensity was in DG granule neurons and pyramidal neurons in CA2, with lesser 
expression in CA3-4 subfields and Sub. (Fig. 5F-H). Prompted by the cortical cell-type taxonomic similarities we described above, we also analyzed METTL7B expression in the neocortex and found high levels in the large pyramidal neurons of layer 5B (Fig. S5D), such as Betz and Meynert cells in $\mathrm{M} 1 \mathrm{C}$ and $\mathrm{V} 1 \mathrm{C}$, respectively. Interestingly, these subregions/layers and cells are generally among the last to exhibit hallmarks of Alzheimer's disease pathology including the formation of plaques, tangles, and neuronal death (McMenemey, 1940; Wilcock and Esiri, 1982; Hof and Morrison, 1990; Davies et al., 1992; Jin et al., 2004; Schonheit et al., 2004; West et al., 2004; Ohm, 2007; Braak and Del Trecidi, 2015). Immunostaining of adult hippocampal and neocortical tissue sections of rhesus macaque, a widely studied Old World monkey, revealed staining profiles comparable to those of humans, with hippocampal DG, CA2 and subicular pyramidal neurons, as well as neocortical large L5B pyramidal neurons, displaying strong immunolabeling for METTL7B (Fig. S5E-F). By contrast, we observed very little expression of METTL7B in corticocortical pyramidal neurons of neocortical and entorhinal L5A and upper layers (L2-4), as well as hippocampal CA1 pyramidal neurons (Fig. 5G, Fig. S5C-E), which are known to be selectively vulnerable in aging and the initial stages of Alzheimer's disease in humans (McMenemey, 1940; Wilcock and Esiri, 1982; Hof and Morrison, 1990; Davies et al., 1992; Jin et al., 2004; Schonheit et al., 2004; West et al., 2004; Ohm, 2007; Braak and Del Trecidi, 2015). We also found that the

308 homolog of $M E T T L 7 B$ is not expressed in adult mouse brain (Fig. S5F-I), indicating that METTLB's expression and function in the adult brain is species-specific.

\section{METTL7B interacts with Alzheimer's disease-related proteins}

312 To identify METTL7B interacting proteins, we performed unbiased proteomic analysis using two 313 different affinity-based approaches to find METTL7B interacting partners. The first approach 
314 utilized HaloTag fusion protein technology (Fig. S6A) and has scarce non-specific binding (Hook,

315 2014). We created stable cell lines by transducing a human cortical neural progenitor cell line,

316 which has been previously utilized to model Alzheimer's disease-related molecular processes

317 (Choi et al., 2014), to express either HaloTag or METTL7B-HaloTag fusion protein. Captured

318 proteins, representing proteins putatively interacting with METTL7B-HaloTag, were detected by

319 silver stain (Fig. S6B) and analyzed by LC-MS/MS (Table S3). We used co-immunofluorescence

320 to observe a high degree of overlap of METTL7B with CALNEXIN and ADFP, markers of the

321 ER and lipid droplets (LD), respectively (Turro et al., 2006) (Fig. S6C). Using Significance

322 Analysis of INTeractome (SAINT) (Choi et al., 2011), we identified 275 true METTL7B

323 interactors (Fig. S6D, Table S4). Fold-enrichment analysis for major subcellular compartments

324 revealed these true METTL7B interactors showed significant enrichment in ER- and LD-

325 associated proteins (Fig. S6E). KEGG pathway enrichment analysis revealed the highest

326 enrichment in the proteasome, protein processing in the ER, oxidative phosphorylation, and three

327 neurodegenerative diseases: Parkinson's disease, AD, and Huntington's disease (Fig. S6F).

We next pursued an overlapping approach using BioID technology, which utilizes biotin ligase (BirA) activity to biotinylate proteins in the vicinity of a protein of interest and better capture interactions that may be weak or transient (Fig. S6G-H, Table S3) (Roux et al., 2012). Here again, METTL7B interactors co-localized with molecular markers for the ER and lipid droplets (Fig. S6I). We identified 1794 true METTL7B interactors that were enriched in ER and lipid dropletsassociated proteins (Fig. S6J-K, Table S4). Molecular pathways involving true interactors were

334 related to endocytosis, SNARE interactions in vesicular transport, and protein-protein processing in ER (Fig. S6L). Moreover, several putative interacting proteins associated with METTL7B have 
protein (APP), inhibition of $\gamma$-secretase (RTN3 and RTN4/NOGO), amyloid binding (NAE1, LRP1, APBB1), protein (de)phosphorylation (PPP3CA, UQCRFS1, CDK5, CALM1, EIF2AK3), APP proteolytic cleavage (ADAM17, CASP3), mitochondrial function (COX6B1, CYCS, ATP5B), calcium homeostasis (ITPR1, ITPR2), ER stress monitoring (ATF6), and protein folding (PDIA3). We confirmed that many of these genes were extensively co-expressed with METTL7B in several hippocampal populations (Fig. S7).

Intersecting the lists of putative METTL7B interacting proteins from both strategies, we found 100 high-confidence proteins, with the most enriched gene ontology term being protein processing in ER (Fig. 6A-B). To further explore the functional significance of the METTL7B interaction network, we examined the profile of spatial overlap between METTL7B true interactors and all KEGG pathways in protein-protein interaction networks, and evaluated the significance of overlap as a function of network distance (Fig. 6C-D). We found that the highestconfidence METTL7B true interactors overlap with several neurodegenerative diseases curated in KEGG pathways in the most proximal network distance from METTL7B, including KEGG Alzheimer's Disease Pathway and Protein Processing in ER (Fig. 6E). We subsequently used immunoblots to confirm that candidate proteins RTN4, APP, and LRP1 were specific to METTL7B sample eluates. RTN3 was not detected by immunoblot in any of the samples, possibly due to low pull-down amounts that were below Western blot detectability threshold (Fig. 6F-G). We next sought to determine whether these proteins were potential substrates for METTL7B methyltransferase activity. To do so, we incubated purified recombinant proteins in a continuous enzyme-coupled S-adenosylmethionine (SAM) methyltransferase assay, which responds fluorescently to S-adenosylhomocysteine, a byproduct of SAM methyltransferase activity. All four assayed samples in which candidate proteins were incubated with recombinant METTL7B 
360 produced a significant increase in signal compared to candidate proteins incubated alone (Fig. $6 \mathbf{H})$.

361 These results suggest that METTL7B uses SAM as a methyl donor, and that METTL7B may act

362 enzymatically on proteins specifically related to Alzheimer's disease pathology.

\section{METTL7B reduces amyloid beta generation}

365 Given the protein interactions and potential activity of METTL7B on APP, we next sought to 366 determine whether METTL7B has an effect on APP proteolytic processing, which plays an 367 important role in Alzheimer's disease pathology (Hardy and Selkoe, 2002; Tanzi and Bertram, 2005; Ballatore et al., 2007). We therefore transduced N2a cells, a mouse neuroblastoma cell line that does not natively express endogenous Mettl7b (Fig. 7A), to express full length human APP 695 along with METTL7B or EGFP. As determined by an enzyme-linked immunosorbent assay (ELISA), METTL7B expression resulted in a $\sim 31.8 \%$ mean reduction in $A \beta 40$ and $\mathrm{a} \sim 34.9 \%$ mean reduction in $A \beta_{42}$ levels, with no significant change in $A \beta_{42} / A \beta_{40}$ ratio (Fig. 7B-C). However, we found that the availability of METTL7B for putative interactions with Alzheimer's diseaseassociated proteins may be limited in conditions with high levels of lipids, as supplementing cell culture media with linoleic and oleic acid complexes resulted in the increased formation of lipid droplets along with a commensurate shift of METTL7B from the ER to lipid droplets, but not METTL7B-interacting and Alzheimer's disease-associated proteins (Fig. 7D-E).

\section{DISCUSSION}

Here we present a detailed single cell transcriptomic analysis of cells in the adult human hippocampal-entorhinal system, and through analysis of this resource describe novel biology related to the molecular diversity of these cells. We found region-specific distinctions in gene 
expression patterns, particularly of excitatory neurons, with clear implications for hippocampalentorhinal physiology.

These data and accompanying analyses refine our understanding of the evolution of allo, meso-, and neo-cortex. The transcriptomic signatures we developed strongly suggest homology between mammalian allocortex and specifically deep layers of the EC and neocortex. Analyses of the transcriptome also suggest that adult neurogenesis in the DG is, if present, extremely limited, with no clear signatures of newborn or immature excitatory neurons present. Although this is not necessarily in conflict with previous efforts, some of which have detected newborn neurons in some but not all adult human hippocampal specimen (Spalding et al., 2013), our analysis of both DCX RNA and protein suggest further analyses is necessary to discern the neurogenic capacity of the adult human hippocampus.

In addition, we implicated METTL7B in aging and disease processes of the human hippocampus. We found strong evidence that METTL7B is enriched in subregions/layers and cell types known to be less vulnerable to initial Alzheimer's disease neuropathology in the HIP, EC and primary neocortical areas. We also found that METTL7B interacts with important Alzheimer's disease-related proteins (e.g., APP, LRP1, RTN3, and RTN4), and that its overexpression reduced $A \beta$ levels. In addition, our results also suggest that the subregion/cell type enrichment we observed could be Catarrhini (i.e., the Old World monkeys and the apes)-specific, supporting the contention that manifestation of some $A \beta$ plaque pathology may have distinct manifestation in primates (Rapoport, 1989). Moreover, brain regions and circuits preferentially affected in early stages of Alzheimer's disease are generally expanded in humans, other apes, and Old World monkeys (Stephan, 1975; Demeter et al., 1990; Morrison and Hof, 1997; Suzuki and Amaral, 2004; Braak and Del Trecidi, 2015), and aged macaques and great apes exhibit early signs of Alzheimer's 
406 disease-like amyloid and Tau pathology (Perez et al., 2013; Finch and Austad, 2015; Edler et al., 407 2017; Paspalas et al., 2018).

Our investigation of METTL7B may also lend support to evidence suggesting the role of

409

410

411

412 several classes of lipids and lipid droplets in Alzheimer's disease pathogenesis. This evidence stems from knowledge of the APOE4 allele, encoding a cholesterol carrier, that is associated with an increased risk for late onset Alzheimer's disease (Strittmatter et al., 1993). In addition, cholesterol dyshomeostasis can directly influence the activity of A $\beta$-related proteases and increase A $\beta$ production (Di Paolo and Kim, 2011), and strong epidemiological evidence supports mid-life hypercholesterolemia as a risk factor for Alzheimer's disease. Moreover, lipid droplet accumulations have been identified in postmortem Alzheimer's disease brains, from the initial report by Alois Alzheimer to more recent reports including a transgenic mouse model of Alzheimer's disease (3xTg-AD) (Alzheimer et al., 1995; Hamilton et al., 2015). Because METTL7B translocates to lipid droplets after fatty acid loading, its availability in the ER and consequently its ability to interact with target proteins implicated in Alzheimer's disease pathogenesis may be diminished. Taken together, the distinct expression pattern of METTL7B in Catarrhini and the integration of METTL7B into mechanisms underlying specific cellular, regional, age, and species-specific aspects of development and disease suggests protein methylation may have previously unappreciated roles in the neurobiology of aging and disease. 
Acknowledgments: We thank K. Meyer for help with gene expression analysis; A. Rosa Campos and K. Motamedchaboki (SBP Medical Discovery Institute, La Jolla, CA) for help with generating proteomics data; M. Horn, A. Huttner, M. Pletikos, and S. Wilson for assistance with tissue acquisition and processing; and J. DeFelipe and the NIH NeuroBioBank, for providing human tissue. We also thank A. Duque for using equipment from MacBrainResource (NIH/NIMH MH113257). This work was supported by the NIH grants DA023999 (P.R.) and MH103339, MH106934, MH110926 and MH109904 (N.S.). The project that gave rise to these results received the support of a fellowship from "la Caixa" Foundation (ID 100010434). The fellowship code is LCF/BQ/PI19/11690010. Additional support was provided by the Kavli Foundation, and the James S. McDonnell Foundation.

Author contributions: D.F. and N.S. conceived and designed the study. D.F. designed and performed most of the METTL7B experiments. J.C. analyzed proteomic data. M.S. prepared nuclei for sequencing. C.X., Q.L., S.M., and G.S. analyzed single nuclei sequencing data. A.T.N.T. performed SAM assay, digital droplet PCRs, and western blots. J.I.A., I.G., L.J.R., and A.M.M.S. contributed to additional data collection. D.F. and N.S. wrote the manuscript. All authors edited the manuscript.

Competing interests: Authors declare no competing interests.

Data and materials availability: All scripts are available to investigators upon request. Supplement contains transcriptome analysis and proteomic data with analysis. Correspondence should be directed to: nenad.sestan@yale.edu 


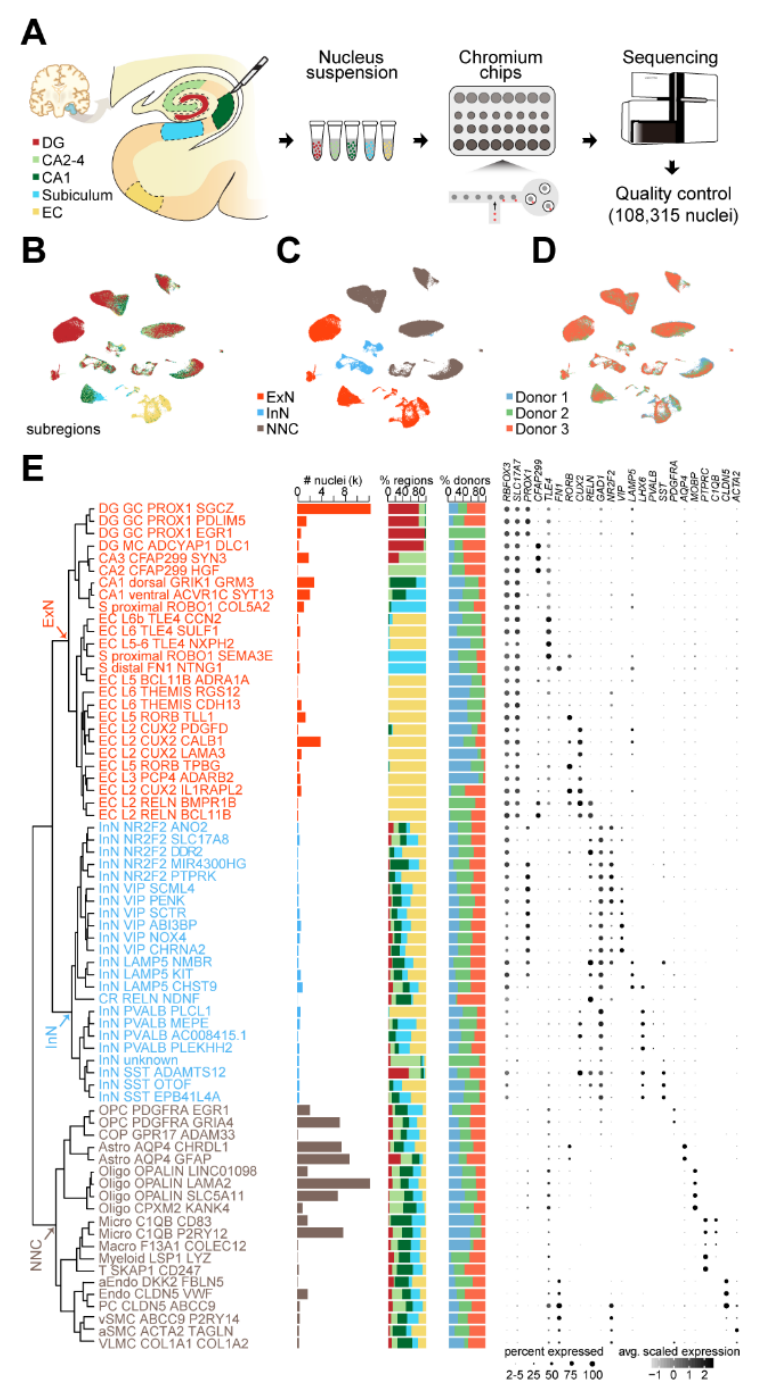

Figure 1. Cell type diversity in the hippocampal-entorhinal system revealed by single-nucleus RNA-seq.

A, Scheme outlining the snRNA-seq procedure including subregion dissections of hippocampus and entorhinal cortex, nucleus isolation and capture, RNA sequencing and quality control. B-D, Uniform Manifold Approximation and Projection (UMAP) visualization representing the transcriptomic arrangement of all nuclei, colored by different subregions (B) major cell types (C), and donors (D). ExN, excitatory neurons; InN, inhibitory neurons; NNC, non-neuronal cells. E. Dendrogram depicting the hierarchical taxonomy across all cell subtypes. Bar plots show the number of nuclei, subregional and donor contributions within each subtype, with coloring scheme conforming to panel c. Dot plot demonstrates the expression of specific marker genes along the cell-type taxonomy. The size and color of dots indicate the percent of expressed nuclei and the average gene expression within each subtype, respectively. GC, granule cell; MC, mossy cell; OPC, oligodendrocyte precursor cell; COP, committed oligodendrocyte precursor cell; Astro, astrocyte; Oligo, oligodendrocyte; Micro, microglia; Macro, macrophage; Myeloid, myeloid cell; T, T cell; aEndo, arterial endothelial cell; Endo, endothelial cell; PC, pericyte; vSMC, venous smooth muscle cell; aSMC, arterial smooth muscle cell; VLMC, vascular and leptomeningeal cell. See also Figure S1 and S2. 
A
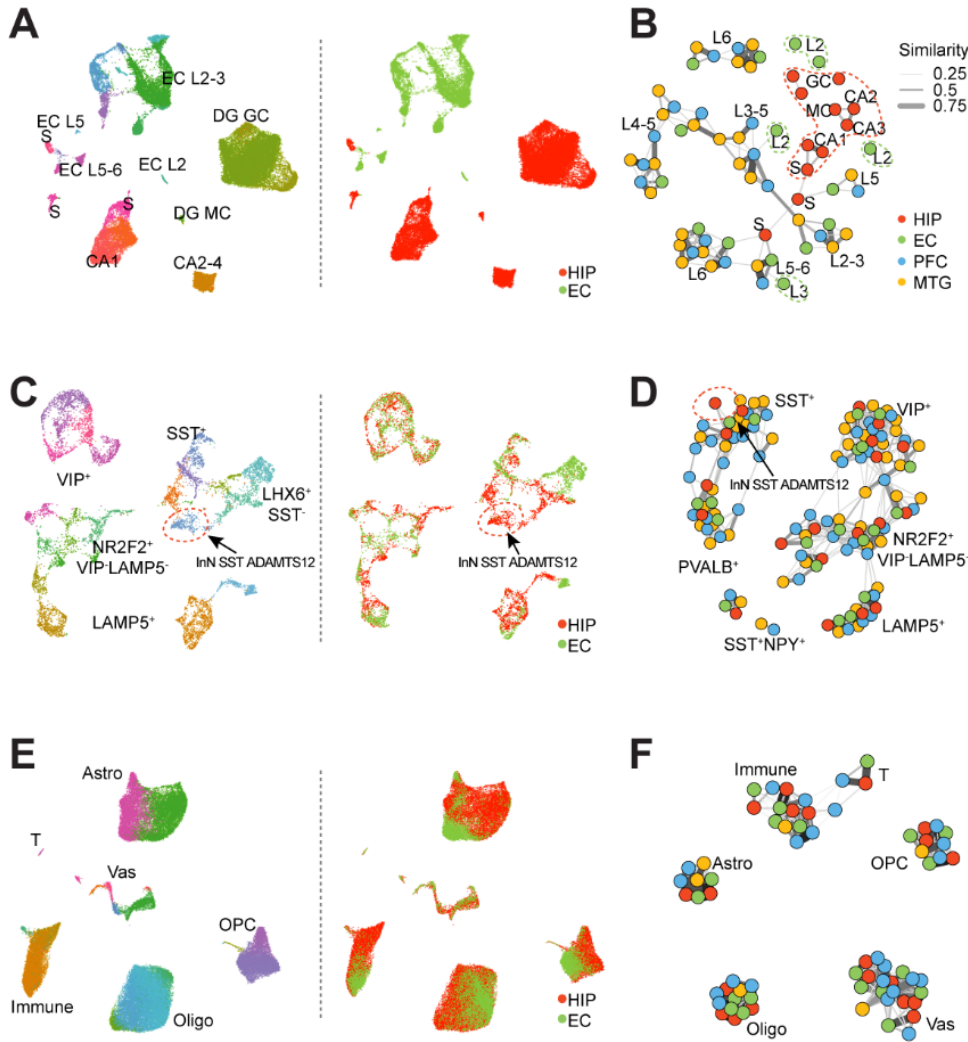

$\mathbf{F}$
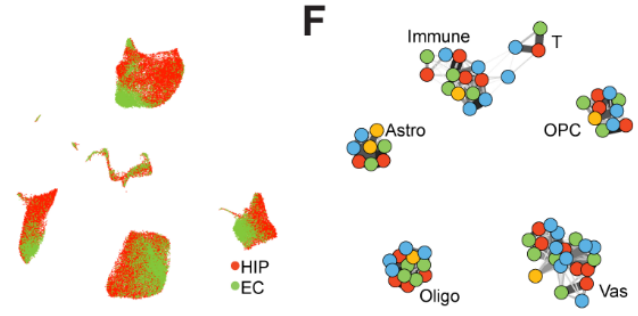

Figure 2. Transcriptomic distinction and similarity of hippocampal and entorhinal cell types. A, Left: UMAP embedding showing all excitatory neuronal subtypes detected in the hippocampalentorhinal system, with naming conventions as in Figure 1. Right, as in left panel, but spatially colored according to two major segregated regions. HIP, hippocampus formation including dentate gyrus (DG), CA1-4 and subiculum (S); EC, entorhinal cortex. B, Network demonstrating the extent of transcriptome similarities among excitatory neuronal subtypes of HIP, EC, medial temporal gyrus (MTG) and dorso-lateral prefrontal cortex (dlPFC). Dots represent the subtypes within each brain region and the widths of lines represent the strength of similarity. Region, subregion and subtype information is indicated by colors and notes. Distinct subtypes of HIP and EC were outlined in corresponding colors. $\mathbf{C}-\mathbf{F}$, As in panels $\mathbf{A}-\mathbf{B}$, for inhibitory neurons $(\mathbf{C}, \mathbf{D})$ and nonneuronal cells $(\mathbf{E}, \mathbf{F})$. See also Figure S2. 

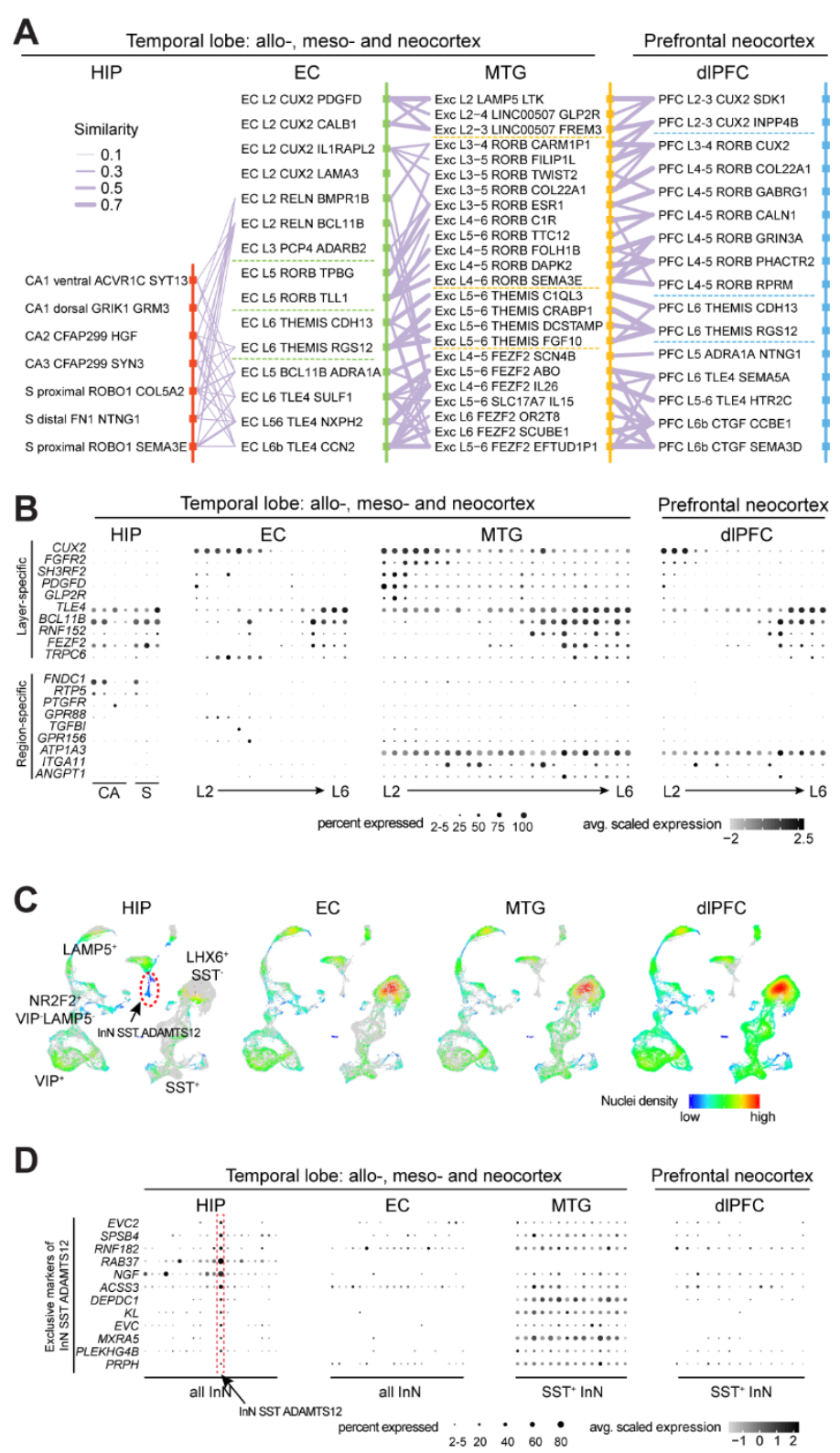

493 A, Transcriptomic relations across subtypes of pairwise regions organized according to layer distributions. The widths of lines denote the strength of the similarities. Broad layer distinction was marked by dotted lines. B, Expression of neocortical upper-layer ( $P D G F D, G L P 2 R, S H 3 R F 2$, FGFR2 and CUX2) and deep-layer markers (TLE4, FEZF2, BCL11B, RNF152 and TRPC6) across subtypes of each region, as well as region-specific genes across these regions. The size and color of dots indicate the percent of expressed nuclei and the average gene expression within each subtype, respectively. C, UMAP layout exhibiting the relative density distribution of nuclei across regions. Nuclei from each region were labeled in rainbow colors to indicate density while nuclei from other regions were colored in grey. Hippocampus-enriched interneuron cluster 'InN SST ADAMTS12' is outlined in the plots. D, Expression of the exclusive markers in cluster InN SST ADAMTS12 across all inhibitory neuron subtypes in HIP and EC and all SST ${ }^{+}$inhibitory neuron subtypes in MTG and dlPFC. See also Figure S3. 

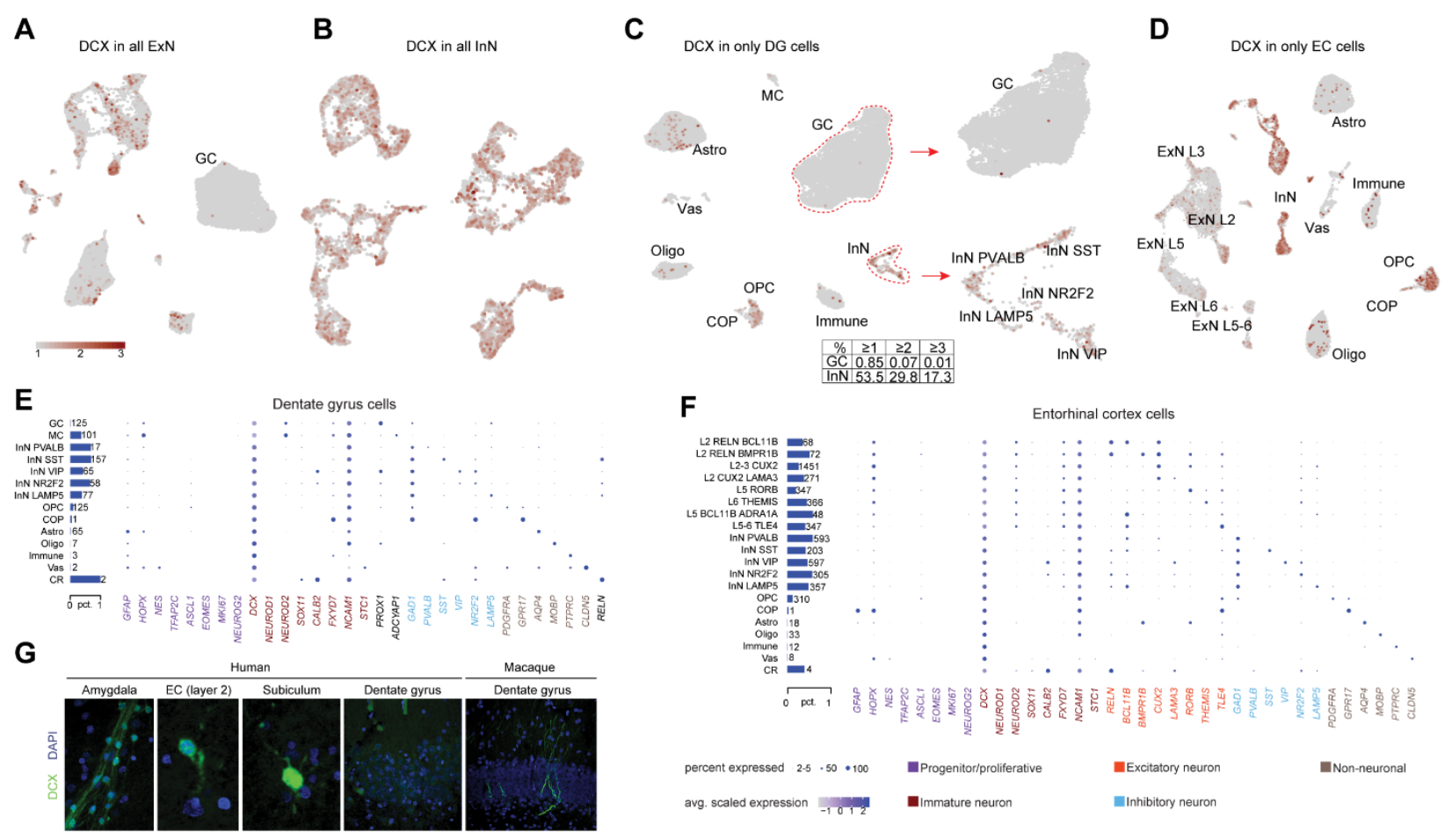

Figure 4. DCX expression in hippocampal and entorhinal cell types.

A, Expression of $D C X$ in all excitatory neurons visualized in UMAP embedding. Granule cells (GC) with sparse $D C X$ expression are noted. B, Expression of $D C X$ in all inhibitory neurons visualized in UMAP embedding. C, $D C X$ expression in only dentate gyrus (DG) cells displayed in UMAP embedding. Major cell type classes are labeled. Zoom-in views illustrate the expression of $D C X$ specifically in four subtypes of inhibitory neurons and GC. Bottom table denotes the percentages of GC and InN expressing $D C X$ over UMI thresholds of 1, 2 and 3. D, $D C X$ expression in only EC cells displayed in UMAP embedding. Cell types expressing $D C X$ (red) are labeled. E, Bar plot shows the numbers and percentages of $D C X+$ cells within each cell type of DG. Dot plot shows the expression of markers of neural stem cells (NSC), neural progenitor cells (NPC), migrating and immature neurons (IM), as well as markers labeling different cell types in $D C X+$ cells of DG. The size and color of dots indicate the percent of expressed nuclei and the average gene expression within each type, respectively. F, Bar plot shows the numbers and percentages of $D C X+$ cells within each cell type of EC. Dot plot shows the expression of markers of neural stem cells (NSC), neural progenitor cells (NPC), migrating and immature neurons (IM), as well as markers labeling different cell types in $D C X+$ cells of EC. The size and color of dots indicate the percent of expressed nuclei and the average gene expression within each type, respectively. $\mathbf{G}$, Images of immunofluorescent cells expressing DCX in regions of human and macaque brain. See also Figure S4 and Table S1. 
A
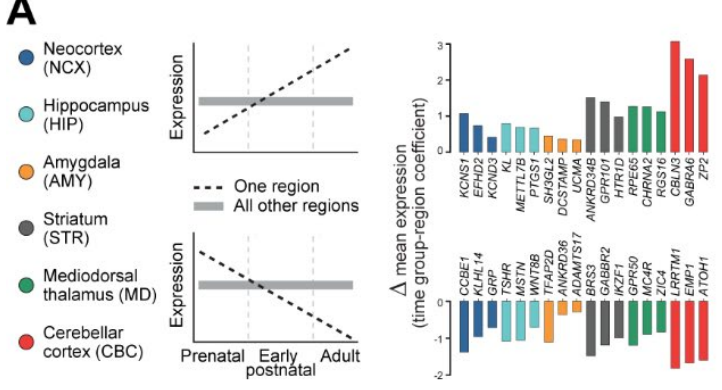

B

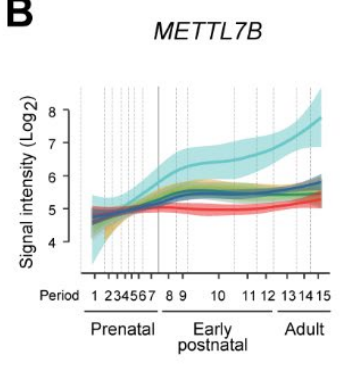

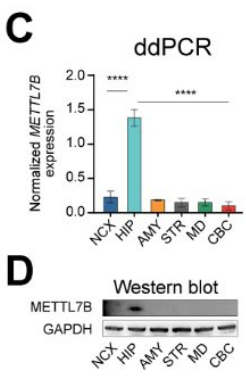

E METTL7B

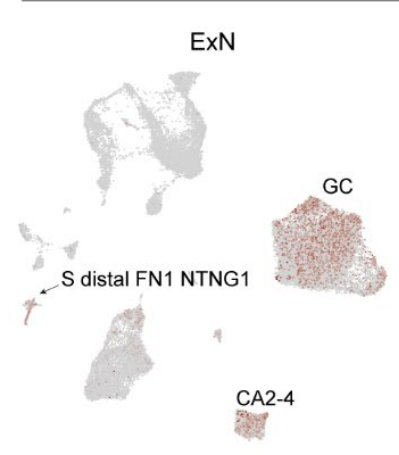

$\ln N$

NNC

$\mathbf{F}$
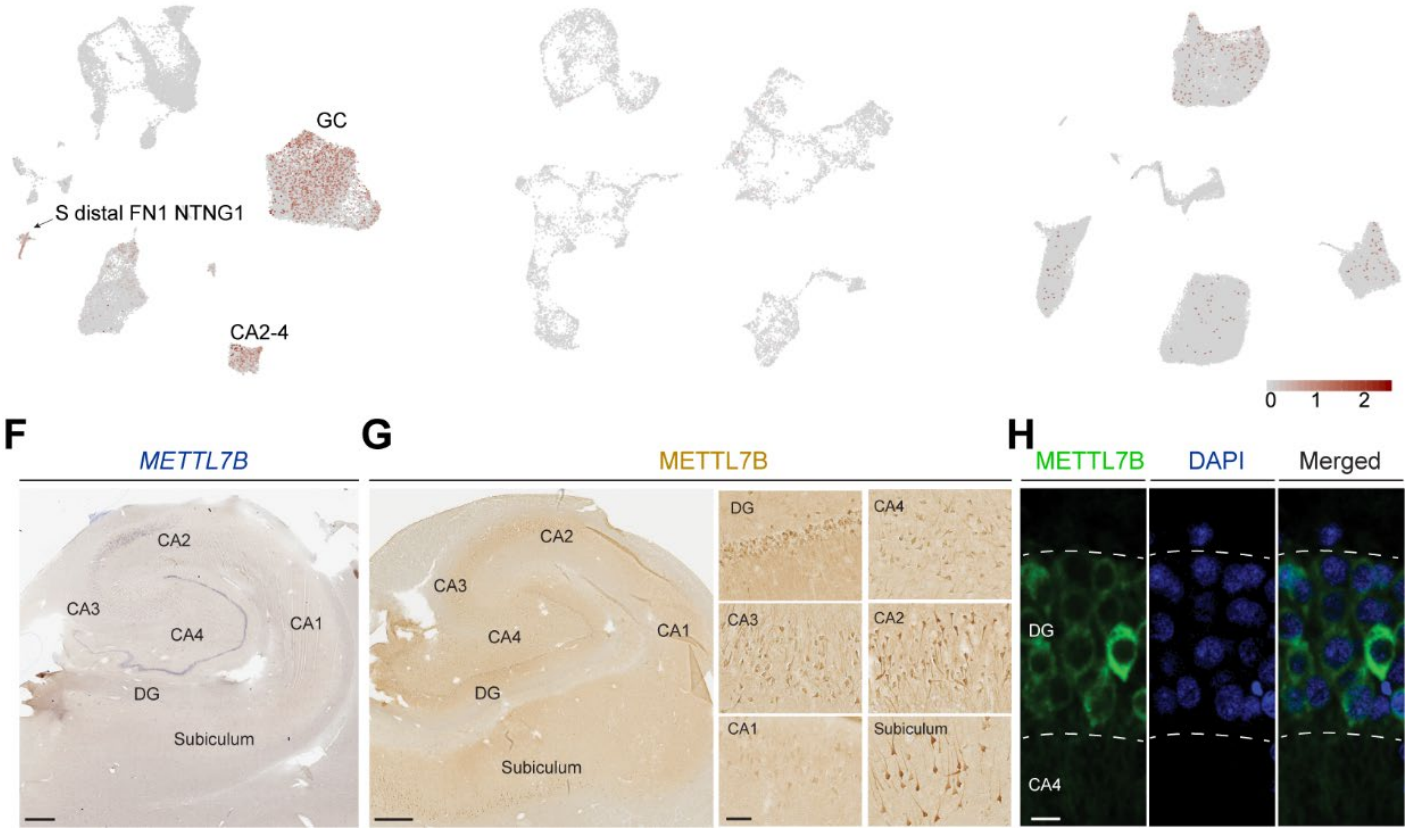

Figure 5. Human METTL7B is enriched in dentate gyrus granule cells.

A, Top three up- and down-regulated genes in six brain regions throughout development. B, Expression trajectory of METTL7B showing enrichment in hippocampus and an increasing expression with age. C-D, Digital droplet PCR and immunoblot validation in six regions of adult brain. One-way ANOVA with post-hoc Dunnett's adjustment ( $* * * * \mathrm{P}<0.0001), \mathrm{N}=3$ per group. E, METTL7B expression in GCs displayed in UMAP embedding. F-H, In situ hybridization and immunostaining of adult hippocampus show prominent labeling of dentate gyrus granule cells, and subicular and CA2 pyramidal neurons. Scale bars $=1 \mathrm{~mm}$; insets $=100 \mu \mathrm{m}$; immunofluorescence $=10 \mu \mathrm{m}$. See also Figure S5 and Table S2. 
A

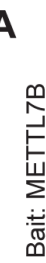

SAINT-identified preys

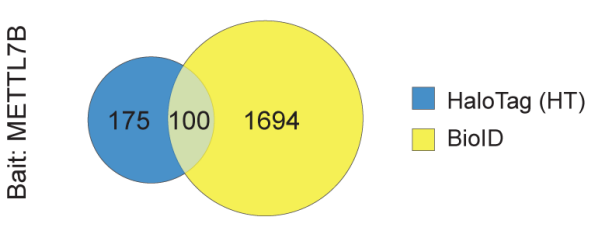

C

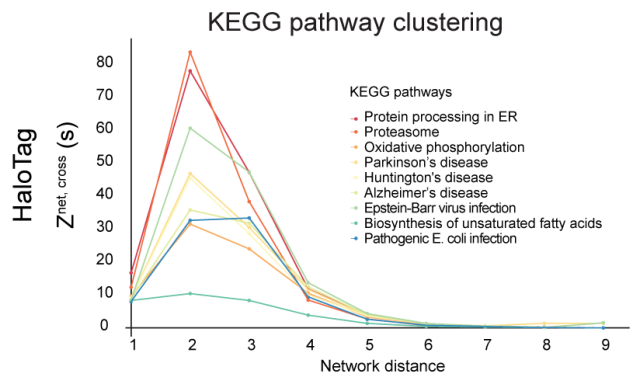

D
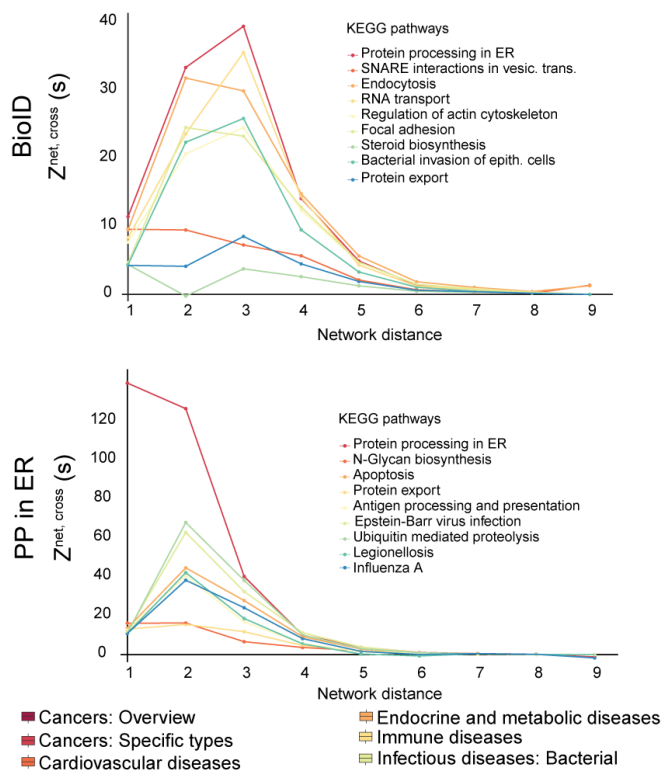

E

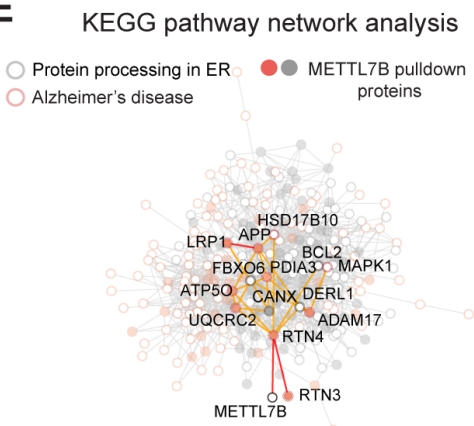

$\mathbf{F}$

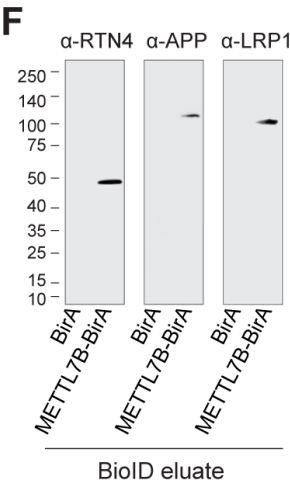

B
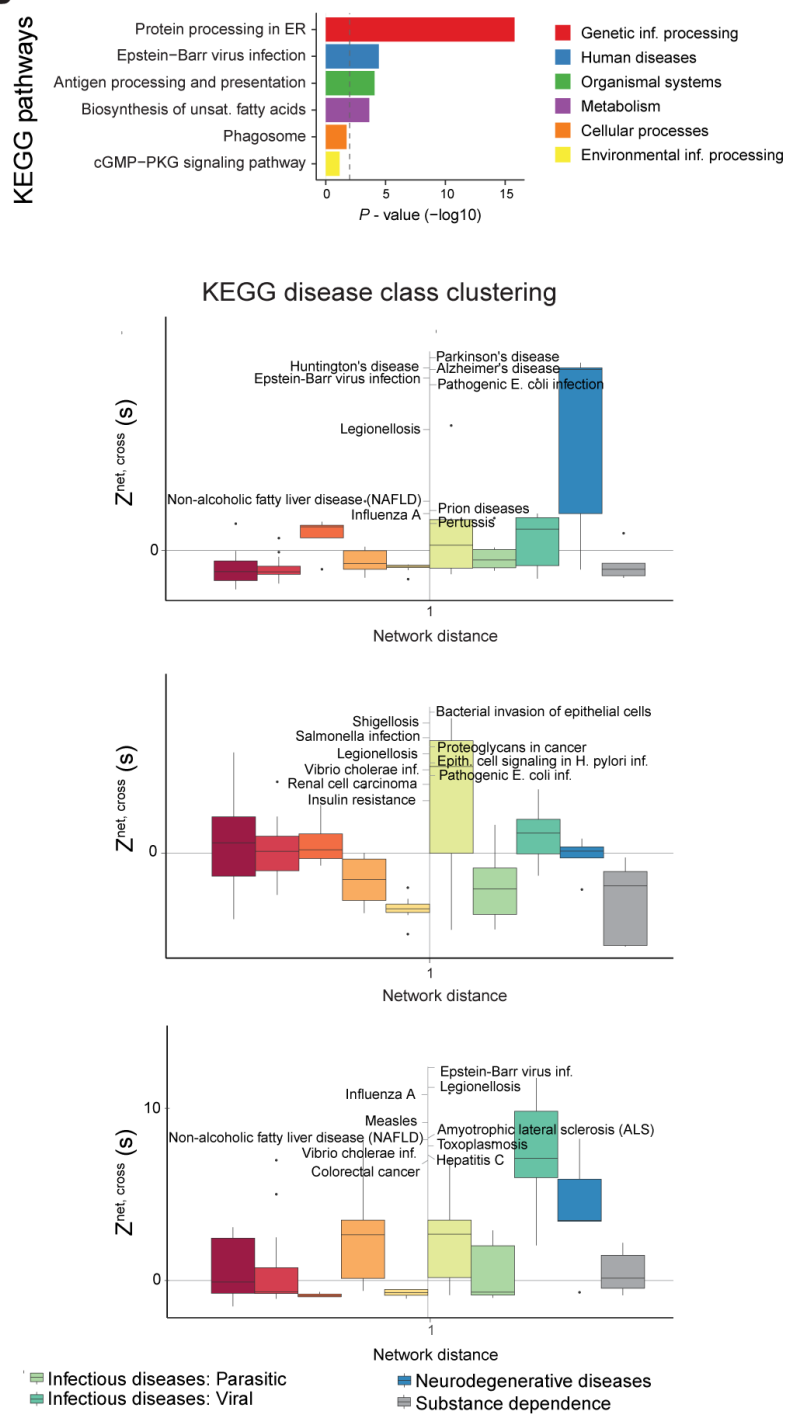

G

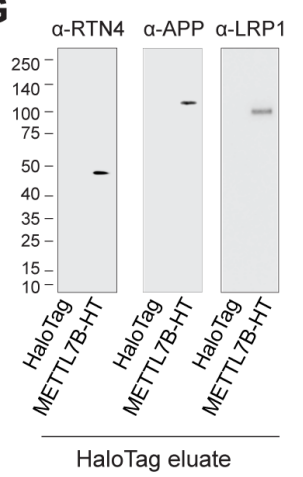

H SAM methyltransferase assay METTL7B

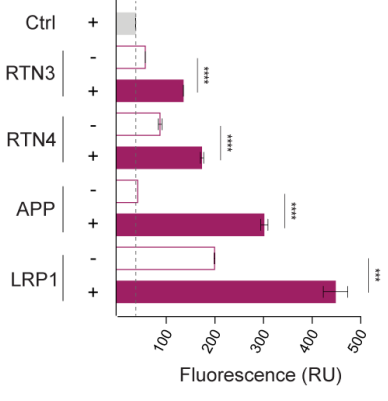
AD.

550 A, Venn diagram of true METTL7B interacting proteins. B, Protein processing in ER (PPER) is the most significantly enriched KEGG pathway of METTL7B interactors. C, Comparing BioID to 
552 HaloTag, the significance of $Z^{\text {net, cross }}$ peak shifts toward a closer network distance (left panel). At 553 the closest network distance, the biggest shift in the significance of $Z^{\text {net, cross }}$ occurs for several 554 neurodegenerative diseases: Parkinson's disease, AD, and Huntington's disease (right panel). D, 555 Validation of spatial clustering analysis using PPER against all KEGG pathways. As expected, 556 PPER is the strongest overlapping KEGG pathway. E, Subset of protein-protein interaction 557 network with all the proteins in the KEGG PPER (grey empty circle) as well as those in 558 Alzheimer's disease pathway (orange empty circle). True METTL7B interactors are shown as 559 filled circles. Top candidate proteins as well as their direct protein-protein interacting partners are 560 highlighted. F-G, Immunoblot confirmation of top candidates. H, SAM-assay showing an increase 561 in methylation in the presence of METTL7B. $P$-values calculated by unpaired two-tailed Student's $562 \mathrm{t}$ test, $\mathrm{N}=3$. All data are mean $\pm \mathrm{SEM}$. $* * * * P<0.0001, * * * P<0.001$. See also Figures $\mathrm{S} 6$, $\mathrm{S} 7$, and 563 Tables S3 and S4.

564

565

566

567

568

569

570

571 
A

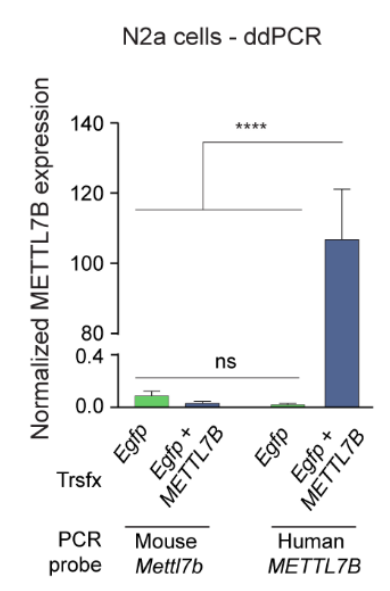

B

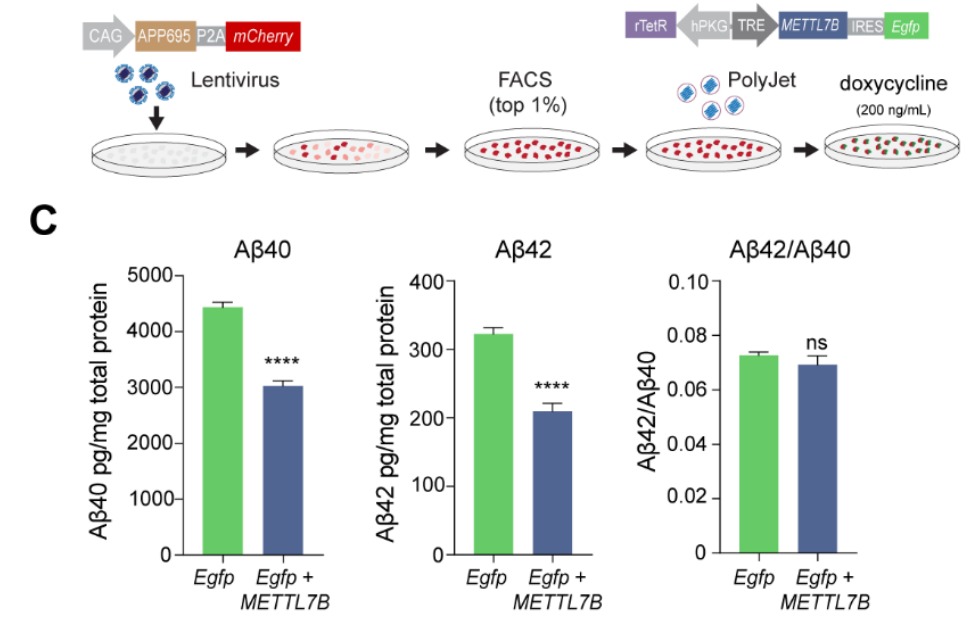

D

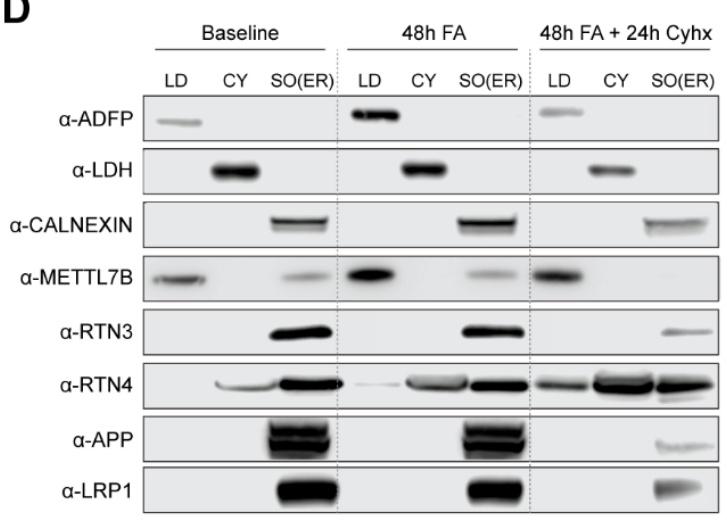

E

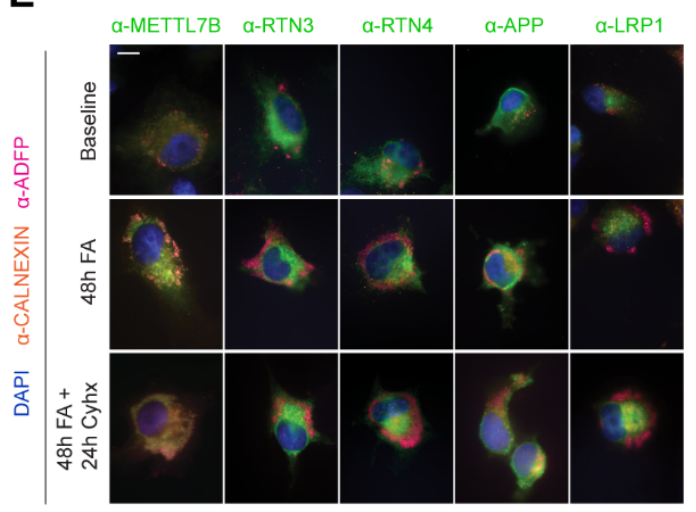

\section{Figure 7. METTL $7 B$ reduces $A \beta$ generation.}

A, ddPCR of human METTL7B misexpression in Neuro-2a cells. One-way ANOVA with posthoc Dunnett's adjustment, $\mathrm{N}=3$ per group. $\mathbf{B}$, Schematic of experimental design. C, ELISA for $\mathrm{A} \beta_{40}$ and $\mathrm{A} \beta_{42}$ levels in cells with or without METTL7B. $P$-values calculated by unpaired twotailed Student's t-test, $\mathrm{N}=6$ per group. All data are mean \pm SEM. $* * * * P<0.0001$. D-E, Immunoanalysis of human neural cells. Increased fatty acid load leads to shift of METTL7B from ER to LDs, while Alzheimer's disease-associated high confidence interactors remain unaffected. Blocking translation of new proteins with cycloheximide (Cyhx) suggests a complete shift of METTL7B. Scale bar $=10 \mu \mathrm{m} . \mathrm{CY}=$ cytosol; $\mathrm{SO}=$ sedimentable organelle. 


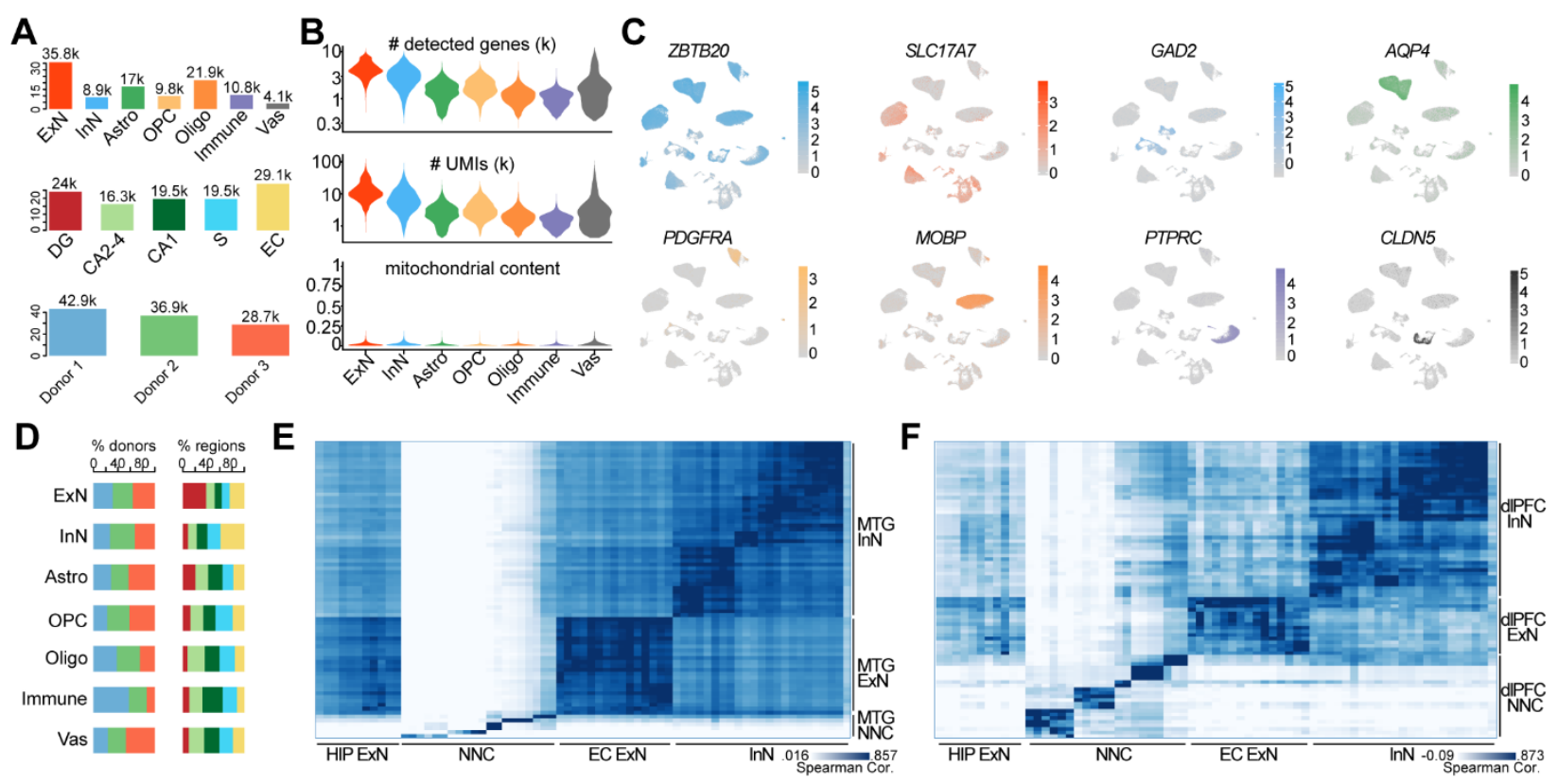

\section{Figure S1 (related to Figure 1). Overview of snRNA-seq data quality.}

A, Bar plots depicting numbers of nuclei passing quality control across major cell types (upper), subregions (middle) and donors (bottom). B, Violin plots denoting the numbers of detected genes (upper), total UMIs (middle) and mitochondrial contents (lower) across major cell types. Note the first two plots were displayed in the logarithmic scale. $\mathbf{C}$, Expression distribution of markers of pan-hippocampus (ZBTB20), excitatory neuron (SLC17A7), inhibitory neuron (GAD2), astrocyte (AQP4), OPC (PDGFRA), oligodendrocyte (MOBP), immune cell (PTPRC) and endothelial cell comprising most vasculatures $(C L D N 5)$ projected onto the UMAP embedding. D, Bar plots showing donor and subregional compositions in each major cell type, with coloring scheme conforming to panel A. E-F, Transcriptome alignments between subtypes of hippocampalentorhinal system (columns) and human medial temporal gyrus (MTG) (E), and human prefrontal cortex (PFC) (F) (rows). Cell grids in heat maps denote the Spearman correlation coefficients between each pair of subtypes across data sets. 
A

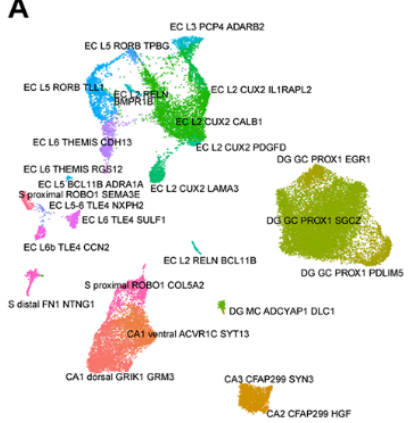

C.

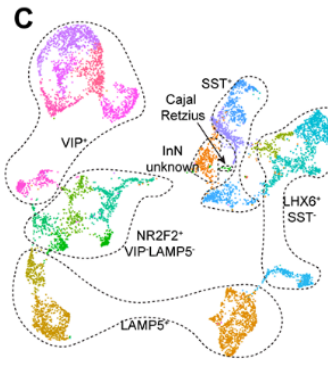

E
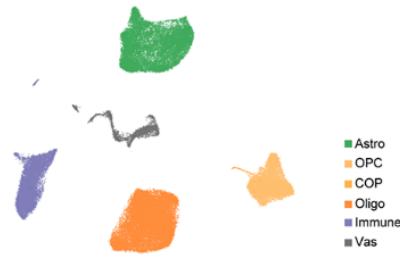

G

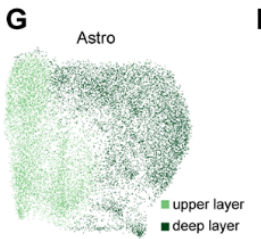

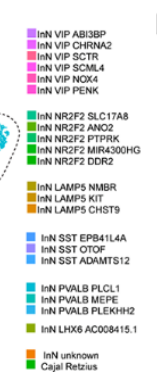

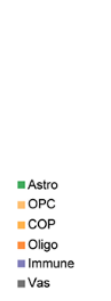

$F$
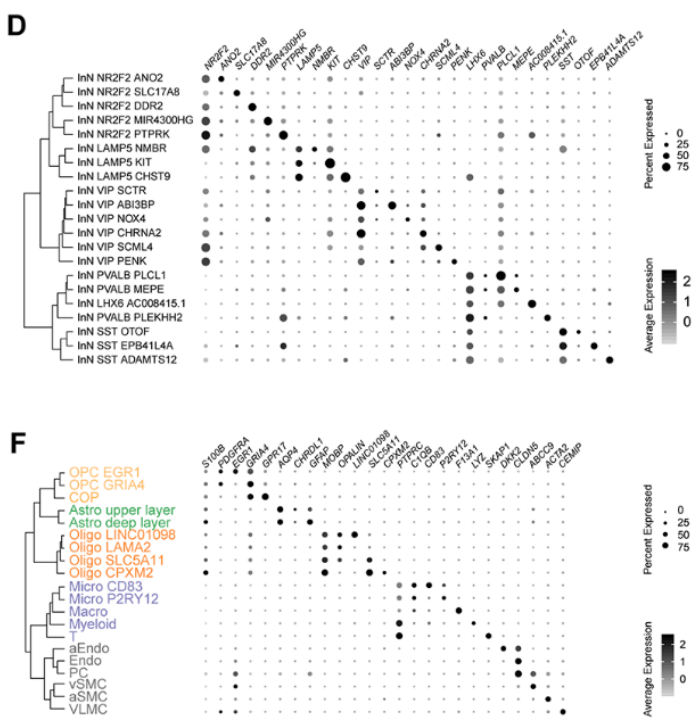

(1)
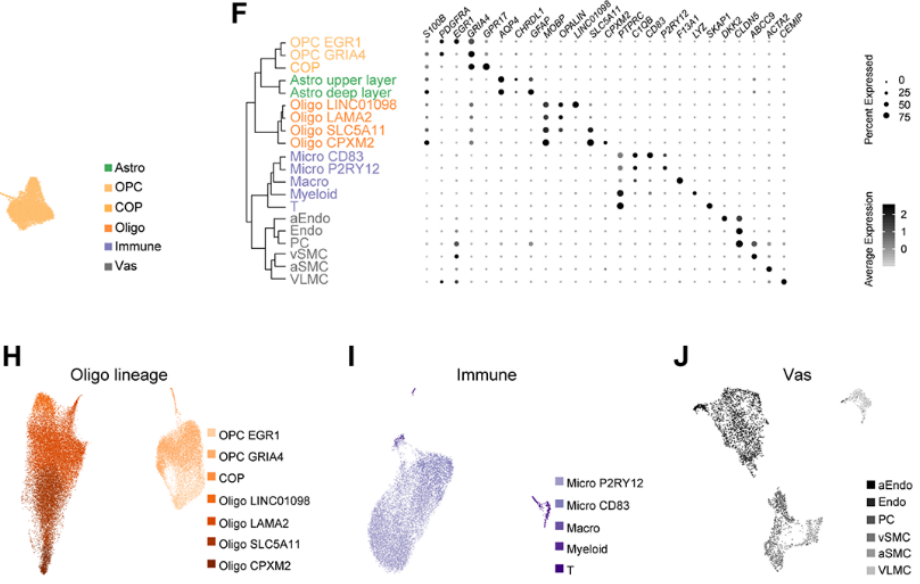

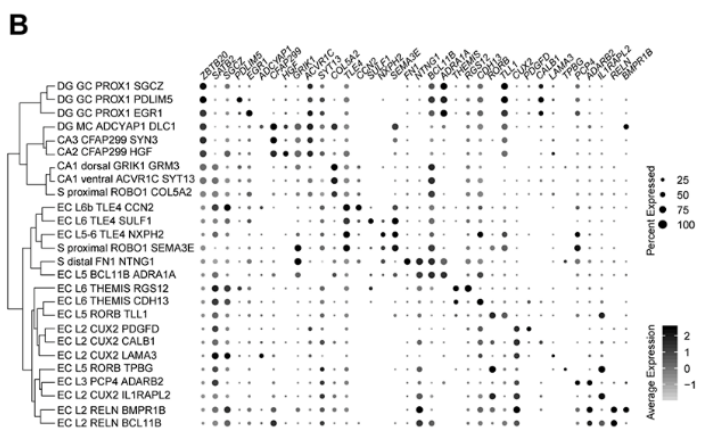

Figure S2 (related to Figures 1 and 2). Transcriptome diversity of hippocampal and entorhinal excitatory neurons.

A, UMAP layout showing all excitatory neuronal subtypes detected in the hippocampal-entorhinal system. B, Hierarchical tree describing the excitatory neuron subtypes and corresponding marker gene expression in them. The size and color of the dot plot indicate the percent of expressed nuclei and the average expression within each subtype, respectively. C, UMAP layout showing all inhibitory neuronal subtypes detected in the hippocampal-entorhinal system. D, Hierarchical tree describing the inhibitory neuron subtypes and corresponding marker gene expression in them. The size and color of the dot plot indicate the percent of expressed nuclei and the average expression within each subtype, respectively. E, UMAP showing all non-neuronal types detected in the hippocampal-entorhinal system. F, Hierarchical tree describing the non-neuronal subtypes and corresponding marker gene expression in them. The size and color of the dot plot indicate the percent of expressed nuclei and the average expression within each subtype, respectively. G-J, UMAP showing subtypes of astrocytes (G), oligodendrocyte lineage (OPCs, COPs and oligodendrocytes) $(\mathbf{H})$, immune cells $(\mathbf{I})$ and vasculature cells $(\mathbf{J})$. 

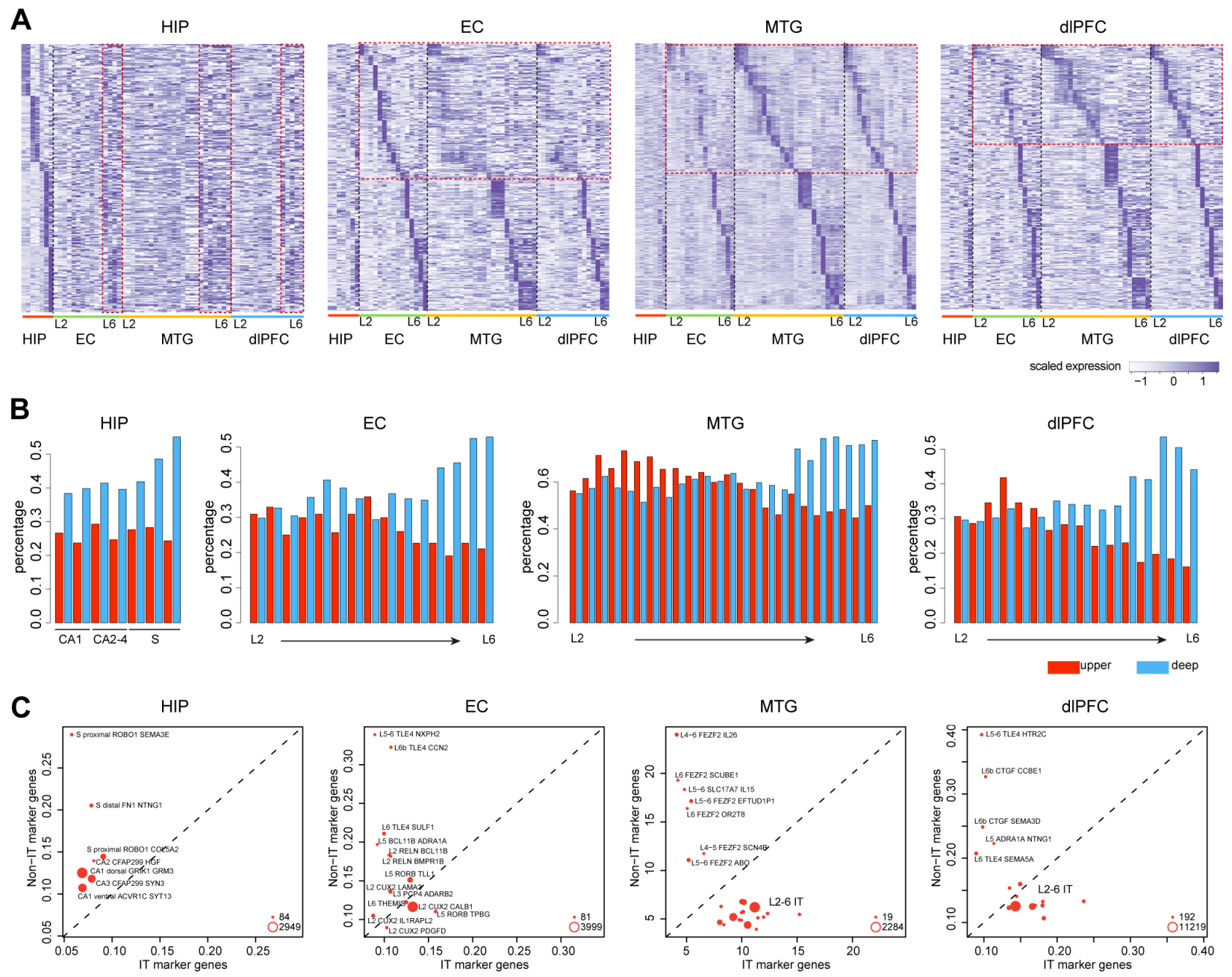

Figure S3 (related to Figure 3). Transcriptome comparison of excitatory neurons between hippocampus, EC and neocortex.

A, Heat map depicting the expression of marker genes from a certain region (rows) across subtypes of all the four regions: HIP, EC, MTG and PFC (columns). The relative expression enrichment of hippocampal marker genes in deep layers of EC, MTG and PFC, and the upper layer divergence between EC and neocortex are outlined. B, Bar plots denoting the percentages of neocortical upper(red) and deep- (blue) layer marker genes that were expressed in each subtype of HIP, EC, MTG, and PFC. C, Scatter plots showing the median expression of intratelencephalic (IT) neuron markers (x axis) versus that of non-IT markers (y axis) in each subtype of HIP, EC, MTG and PFC. Dot size indicates the number of cells within the corresponding subtype. 
A

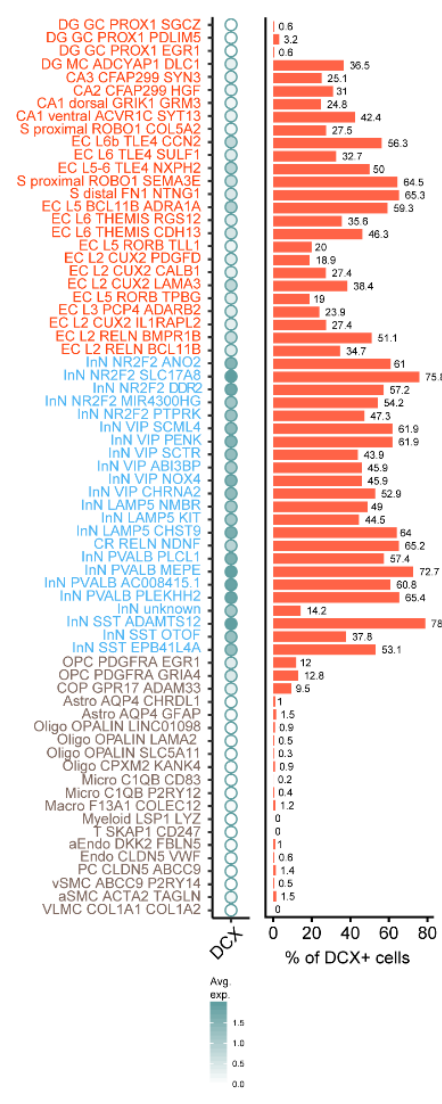

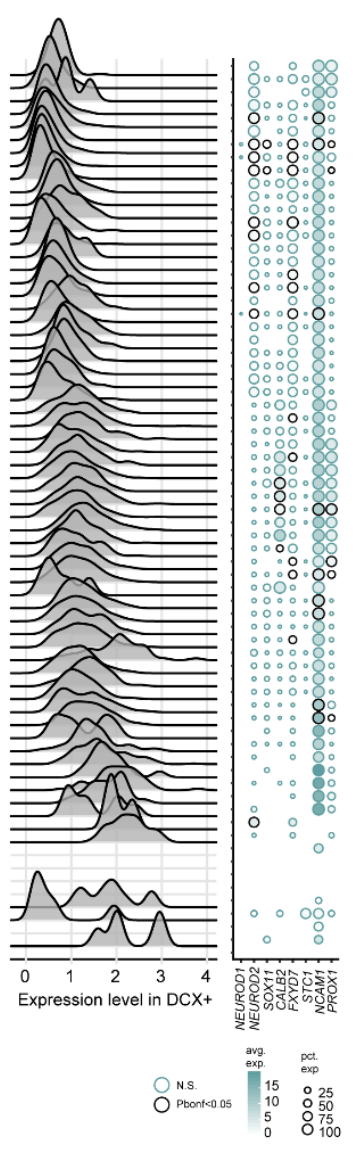

B
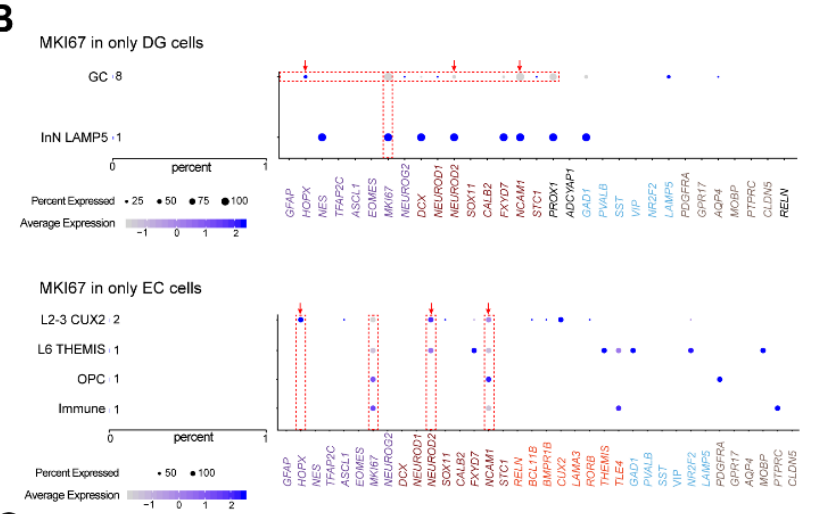

$C^{\text {Averos }}$
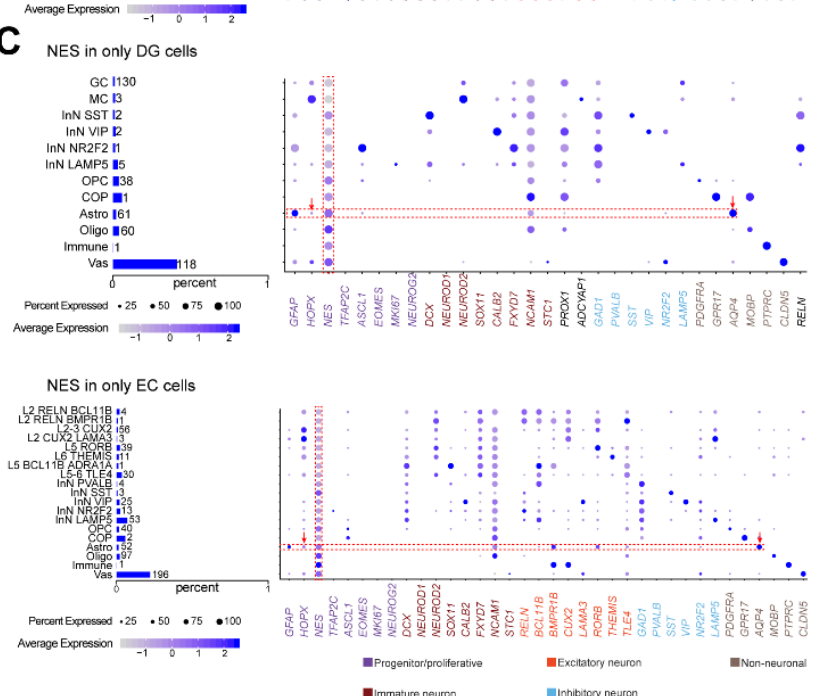

Figure S4 (related to Figure 4). Characterization of $\mathrm{DCX}+, \mathrm{MKI67+}$ and $\mathrm{NES}+$ cells.

A, First panel: Average expression of $D C X$ across clusters. Second panel: percentage of $D C X$ positive cells in each cluster. Third panel: expression (Seurat-log normalized counts) level of $D C X+$ cells. Fourth panel: Average expression (color) and percentage of cells expressing (size) gene markers of migrating and immature granule cells, and PROX1. Black circle indicates significant enrichment of $D C X$ and each other gene colocalization compared to $D C X$ - cells calculated by means of a Fisher's exact test. B, C, Bar plot shows the numbers and percentages of $M K I 67+$ cells $(\mathbf{B})$ or NES + cells (C) within each cell type of DG (upper) and EC (bottom). Dot plot shows the expression of markers of neural stem cells (NSC), neural progenitor cells (NPC), migrating and immature neurons (IM), as well as markers labeling different cell types in MKI67+ or NES + cells. The size and color of dots indicate the percent of expressed nuclei and the average gene expression within each type, respectively. 
A

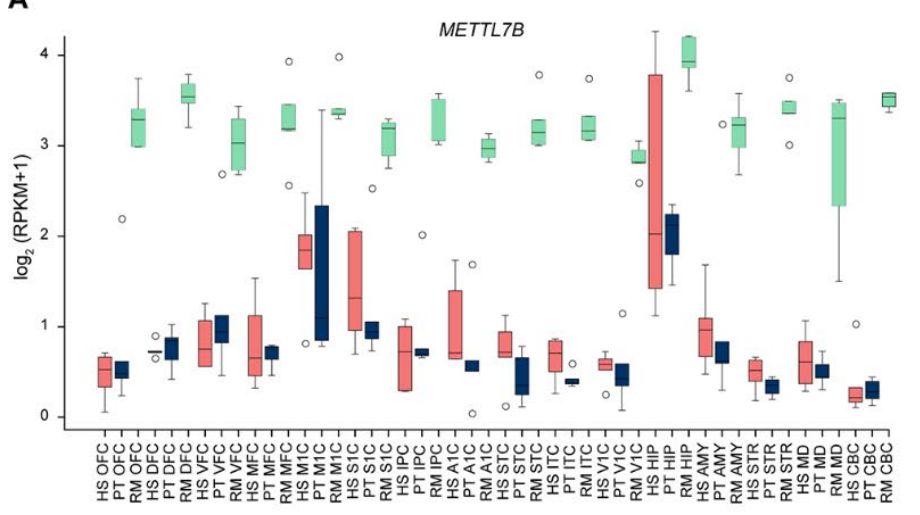

B

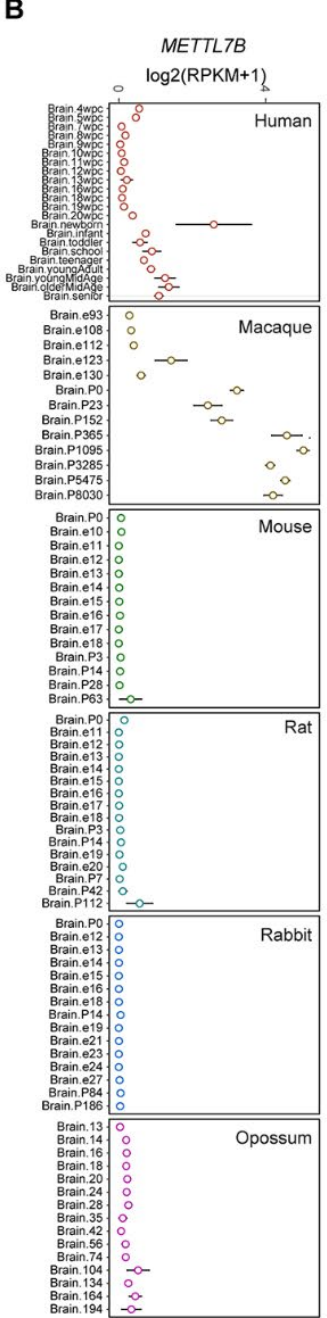

D

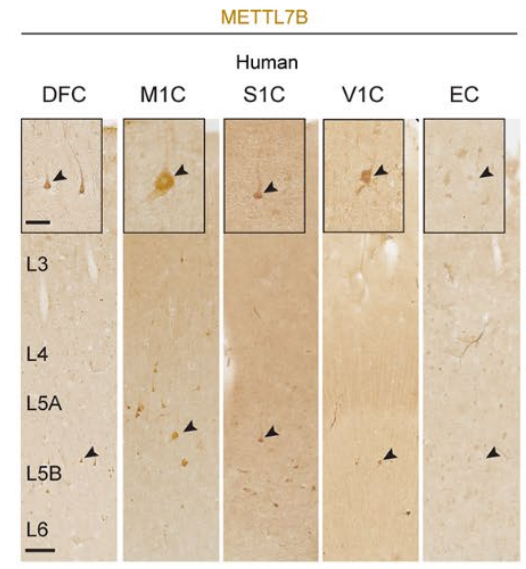

$\mathbf{F}$

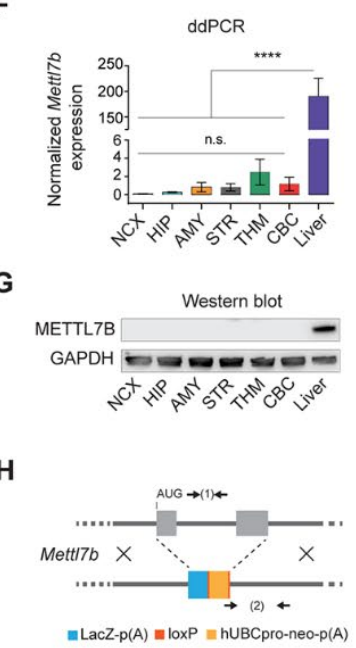

C

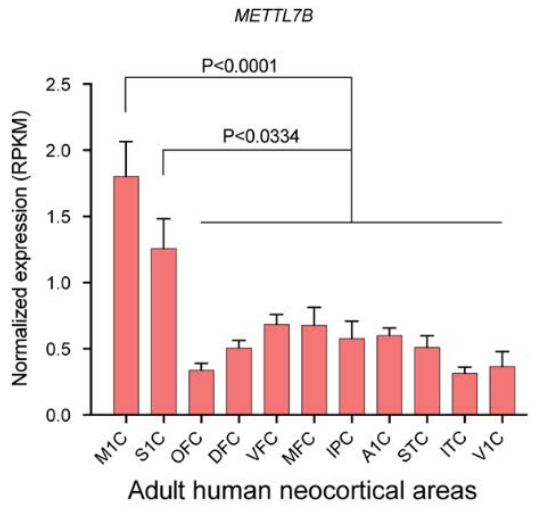

E

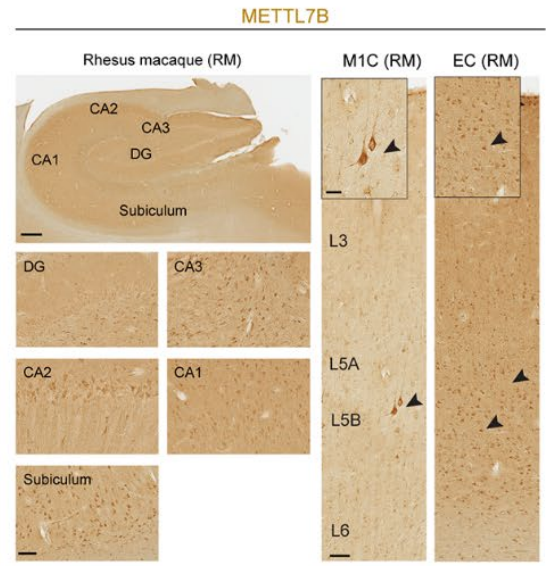

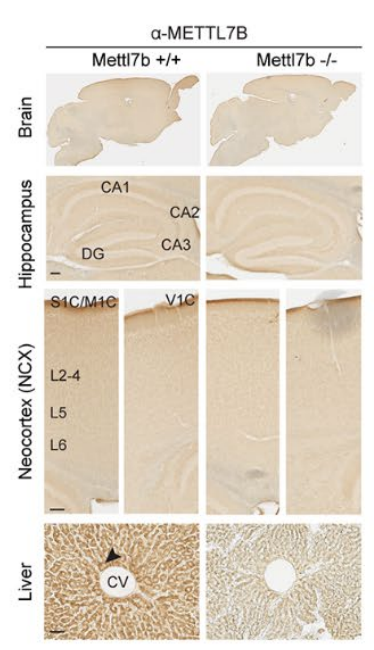

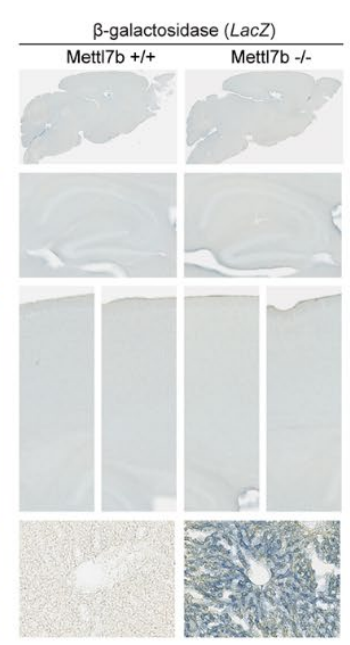

Figure S5 (related to Figure 5). METTL $7 B$ is expressed in primate brain but not mouse brain.

A, Exon-array expression of METTL7B homologs in human (HS), chimpanzee (PT), and rhesus macaque (RM) brain regions. B, RNA-seq expression of $M E T T L 7 B$ in the brain (forebrain/cerebrum) of multiple species at different developmental stages. Expression data was obtained from Cardoso-Moreira et al. 2019. C, RNA-seq expression of METTL7B in human NCX. 
One-way ANOVA with post-hoc Dunnett's adjustment, all groups $\mathrm{N}=6$, except MFC N=5. Data are means \pm SEM. D, Prominent immunolabeling of layer 5B (L5B) pyramidal neurons (arrowheads), including Betz and Meynert cells in M1C and V1C, respectively. Scale bars $=150$ $\mu \mathrm{m}$; insets $=50 \mu \mathrm{m}$. E, METTL7B immunolabeling of hippocampus, Betz cells, and pyramidal neurons in RM brain. Scale bars $=100 \mu \mathrm{m}$; inset $=50 \mu \mathrm{m}$. F, Digital droplet PCR of mouse brain regions and liver, and $\mathbf{G}$, immunoblot validation of Mettl7b expression in the adult mouse brain and liver showing significantly higher expression in liver with no differences between brain regions. One-way ANOVA with post-hoc Dunnett's adjustment ( $* * * * \mathrm{P}<0.0001), \mathrm{N}=3$ per group. All data are mean \pm SEM. H, Generation of Mettl7b knock-out ( $/-)$ mouse. I, Immunostaining reveals Mettl7b protein and lacZ expression in liver. No staining observed in adult mouse brain. Scale bars: brain $=100 \mu \mathrm{m}$; liver $=50 \mu \mathrm{m} . \mathrm{CV}=$ central vein. A1C, primary auditory cortex; DFC, dorsolateral prefrontal cortex (aka DLPFC); EC, entorhinal cortex; IPC, posterior inferior parietal cortex; ITC, inferior temporal cortex M1C, primary motor cortex; MFC, medial prefrontal cortex; OFC, orbital prefrontal cortex; S1C, primary somatosensory cortex; STC, superior temporal cortex; V1C, primary visual cortex; VFC, ventrolateral prefrontal cortex. 
A

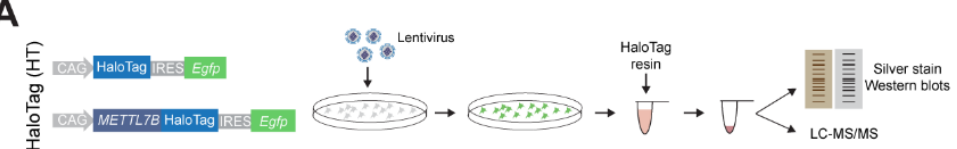

B

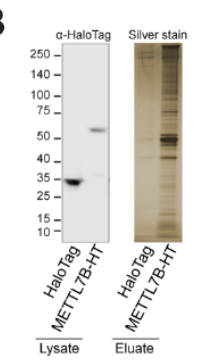

D
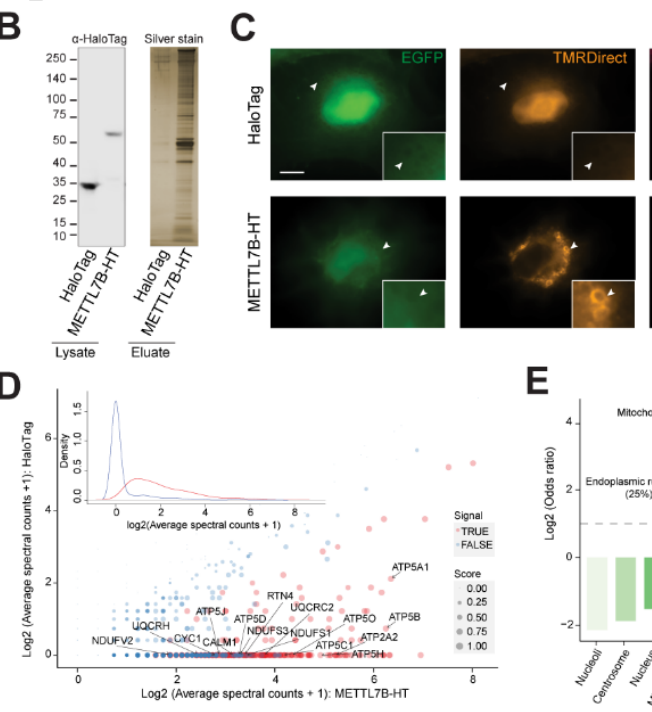

E

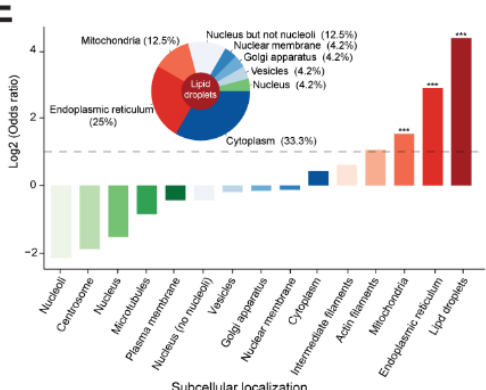

G
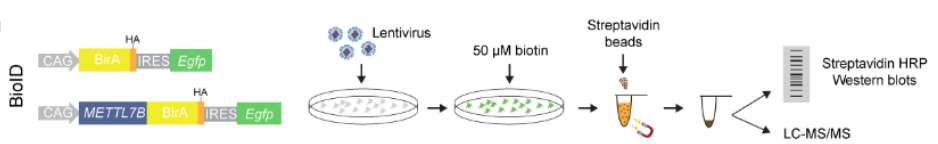

H
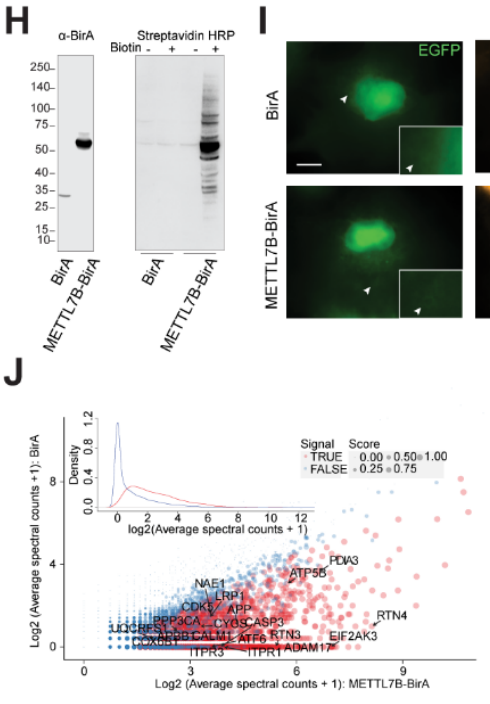
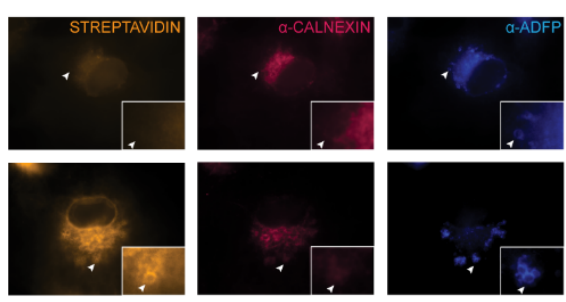

K

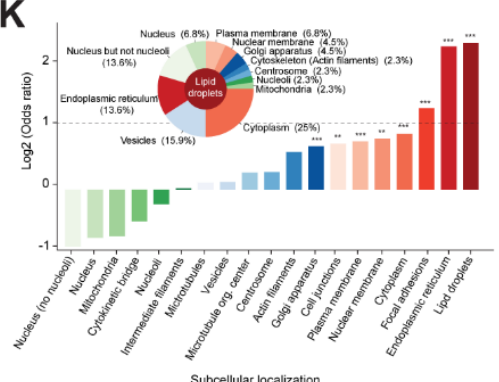

$\mathbf{F}$
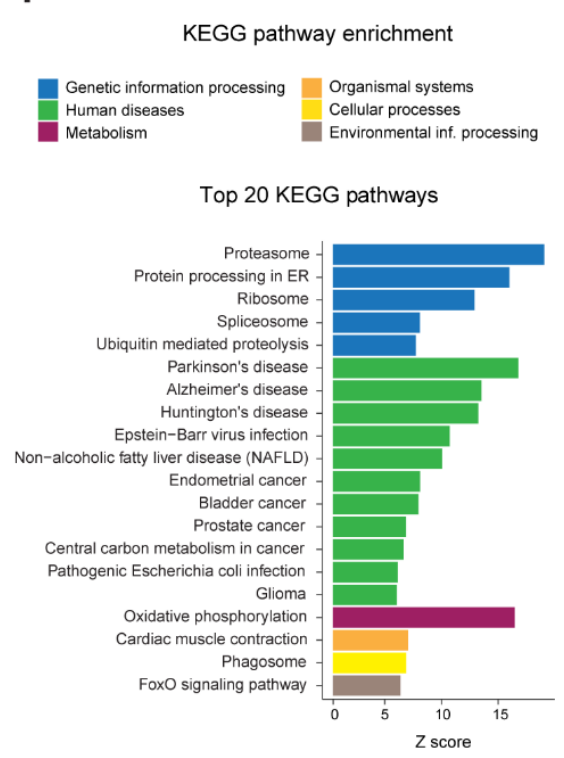

L

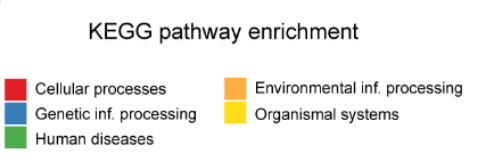

Top 20 KEGG pathways

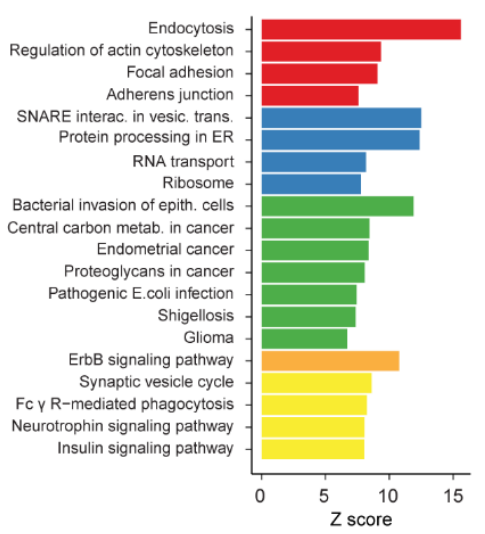

Figure S6 (related to Figure 6). KEGG pathway enrichment of METTL7B interacting proteins.

730 A, Schematic of HaloTag (HT) pulldown design. B, Immunoblot validation of HT proteins and silver stain of pulldown eluates showing more proteins captured in METTL7B-HT sample. C, Immunofluorescence staining showing that METTL7B fusion protein (TMR-Direct) co-localizes with CALNEXIN and ADFP. Scale bars $=10 \mu \mathrm{m}$ D, SAINT analysis distinguishes true METTL7B interactors (red) from false ones (blue) based on MS spectral counts. The figure shows the average 
spectral counts in 3 test experiments (x axis) and 3 control experiments for all detected proteins. The inset clarifies separation between true METTL7B interactors (red curve) and the false ones (blue curve) in terms of spectral count distribution. E, Fold-enrichment test for major subcellular compartments cataloged in Human Protein Atlas database and mammalian cytoplasmic lipid droplet proteomes. The inset shows subcellular composition (\%) of LD associated proteins. $* * * \mathrm{P}<0.001$. F, KEGG pathway enrichment analysis showing molecular pathways involving true interactors are associated with three neurodegenerative diseases: Alzheimer's, Parkinson's, and Huntington's disease. G, Schematic of BioID pulldown experimental design. H, Immunoblot validation of BioID proteins ( $\alpha$-BirA) and pulldown efficiency (STREPTAVIDIN-HRP) after supplementing cell culture media with $50 \mu \mathrm{M}$ biotin for 24 hours. I, METTL7B-expressing cells exhibit vast biotinylation of endogenous proteins (STREPTAVIDIN) which co-localize with CALNEXIN and ADFP, ER and LD markers, respectively. Scale bars $=10 \mu \mathrm{m}$ J, SAINT analysis distinguishes true METTL7B interactors (red) from false ones (blue) based on MS spectral counts. The figure shows the average spectral counts in 3 test experiments ( $\mathrm{x}$ axis) and 3 control experiments for all detected proteins. The inset clarifies separation between true METTL7B interactors (red curve) and the false ones (blue curve) in terms of spectral count distribution. K, Fold-enrichment test for major subcellular compartments cataloged in Human Protein Atlas database and mammalian cytoplasmic lipid droplet proteomes. The inset shows subcellular composition (\%) of LD associated proteins. $* * * \mathrm{P}<0.001, * * \mathrm{P}<0.01$. L) KEGG pathway enrichment analysis. 
bioRxiv preprint doi: https://doi.org/10.1101/2019.12.31.889139; this version posted January 2, 2020. The copyright holder for this preprint (which was not certified by peer review) is the author/funder, who has granted bioRxiv a license to display the preprint in perpetuity. It is made available under aCC-BY-NC-ND 4.0 International license.

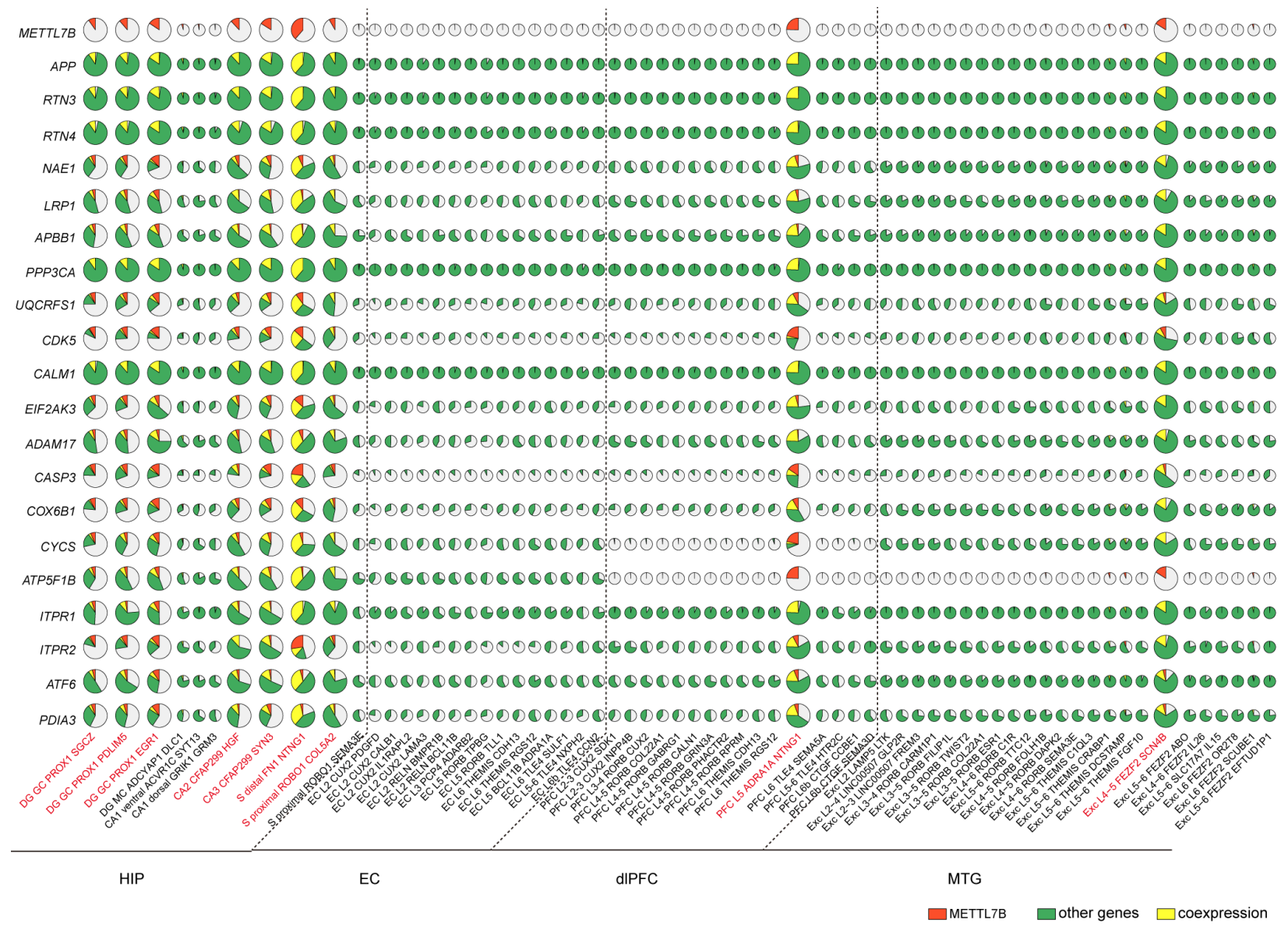
Pie charts showing the percent of cells expressing METTL7B but not certain $M E T T L 7 B$ interactor (red), the percent of cells expressing each of the METTL7B interactors but not METTL7B (green), as well as the percent of cells co-expressing METTL7B and certain interactor (yellow) out of all cells within the subtypes of HIP, EC, MTG and PFC. Each row represents a gene and each column denotes a subtype. 
SUPPLEMENTAL TABLES

796

797 Table S1 (related to Figure 4) | Tissue samples used in adult neurogenesis analyses.

798

799 Table S2 (related to Figure 5) | Complete list of genes exhibiting brain region-specific up-

800 or down-regulated expression.

801

802 Table S3 (related to Figure 6) | LC-MS/MS data from BioID and HaloTag experiments.

803

804 Table S4 (related to Figure 6) | SAINT analysis of LC-MS/MS data from BioID and

$805 \quad$ HaloTag experiments.

806

807 Table S5 (related to STAR methods) | Primer and probe sequences.

808 
809

810

811

812

813

814

815

816

817

818

819

820

821

822

823

824

\section{Methods}

\section{Human, non-human primate tissue}

Human samples were obtained from the collections of the Sestan and Rakic laboratories and from Javier DeFelipe's collection in the Instituto Cajal in Madrid (Spain). Non-human primate brain specimens were obtained from the tissue collection of the Sestan laboratory. All clinical histories, tissue specimens, and histological sections were evaluated to assess for signs of disease, injury, and gross anatomical and histological alterations.

Frozen archival tissue human specimens were used for snRNA-seq. No obvious signs of neuropathological alterations were observed in any of the specimens considered and analyzed in this study. For all other specimens, regions of interest were sampled from frozen tissue slabs or whole specimens stored at $-80^{\circ} \mathrm{C}$. To ensure consistency between specimens, all dissections were performed by the same person. Frozen tissue slabs were kept on a chilled aluminum plate during dissections. EC and four hippocampal subregions (DG, CA 2-4, CA1, and Sub) were microdissected as previously reported (Kang et al., 2011)from fresh frozen post-mortem human brains previously cut into $1-\mathrm{cm}$ thick serial, coronal sections, and snap frozen in isopentane (J. T. Baker).

Fresh tissue specimens for histology were fixed with 4\% paraformaldehyde/PBS followed by $30 \%$ sucrose/PBS. No obvious signs of neuropathological alterations were observed in any of the human, or macaque specimens analyzed in this study. The postmortem interval (PMI) was defined as hours between time of death and time when tissue samples were fresh frozen or started to undergo fixation process.

All human (Homo sapiens) brain specimens (18 Y female, $23 \mathrm{Y}$ male, $23 \mathrm{Y}$ male, $42 \mathrm{Y}$ male, 42 Y male, 50 Y female, 51 Y male, 63 Y male, 69 Y female, 74 Y male, 78 Y female, 79 
832 Y female, 79 Y female, and one case where sex and age are unknown; PMIs up to 24 hours) were

833 de-identified and collected from clinically unremarkable donors and one case that died in status

834 epilepticus. Tissue was collected following the guidelines provided by the Yale Human

835 Investigation Committee (HIC) for the Sestan and Rakic collection or by the European Union for

836 DeFelipe's samples from Spain. Tissue was collected and handled in accordance with ethical

837 guidelines and regulations for the research use of human brain tissue set forth by the NIH

838 (http://bioethics.od.nih.gov/humantissue.html) and the WMA Declaration of Helsinki

839 (http://www.wma.net/en/30publications/10policies/b3/index.html). Appropriate informed consent

840 was obtained and all available non-identifying information was recorded for each specimen.

841

842

843

844

845

846

847

848

849

850

851

852

853

854

All studies using non-human primates were carried out in accordance with a protocol approved by Yale University's Committee on Animal Research and NIH guidelines. The postmortem interval (PMI) was defined as hours between time of death and time when tissue samples were frozen or started to undergo fixation process. Rhesus macaque (Macaca mulatta) brain samples were collected postmortem from 2 adult specimens (4 Y male, 8 Y female; PMIs up to 2 hours). Macaque brains were post-fixed by immersion in $4 \%$ paraformaldehyde/PBS for approximately 24 hours, followed by $30 \%$ sucrose/PBS, and stored at $+4^{\circ} \mathrm{C}$.

\section{Anatomical definition of sampled subregions of the hippocampal formation and entorhinal}

$\underline{\text { cortex }}$

The dentate gyrus (DG) was sampled from the posterior part of the anterior third of the hippocampal formation. It included the suprapyramidal blade, infrapyramidal blade and crest of as well as all three layers: molecular, granular, and polymorphic. The polymorphic layer 
contained only the superficial part, while the deeper part of the hilus of the DG was dissected as part of the proximal portion (nearer DG) of the CA2-4 region.

Cornu Ammonis (CA) 2-4 region was sampled after DG was dissected and contained the remaining part of the hippocampal proper anlage until approximately CA1 region, including all three layers: molecular, pyramidal and stratum oriens. The proximal portion, corresponding to CA4, contained most the tissue of the hilus of the DG.

$\underline{C A 1 \text { region }}$ (Sommer's sector) was sampled from approximately the border of CA2 to the subiculum, comprising the most distal (further away from the DG) portion of cornu Ammonis. The border between CA1 and CA2 is difficult to reliably identify and thus small pieces of the neighboring CA2 and, vice versa, could have been occasionally present in the sample.

The subiculum (S) forms the most distal part of the hippocampal formation that is adjacent to CA1 region. It was sampled adjacent to the CA1 sample and contained all cortical layers and the superficial region of underlying white matter.

The entorhinal cortex $(\boldsymbol{E C})$ spreads over both the gyrus ambiens and a considerable part of the parahippocampal gyrus. The EC samples were collected from the middle portion of the parahippocampal gyrus of the same tissue slab used to dissect the subregions of the hippocampal formation, corresponding to the proper entorhinal subregion and Brodmann area 28. The EC was also defined by presence of numerous wart-like elevations (verrucae hippocampi) on the 
surface of the gyrus. Samples contained all cortical layers and the superficial region of underlying white matter.

\section{$\underline{\text { Brain cell nuclei isolation }}$}

The brain cell nuclei were isolated according to our previous protocol (Li et al., 2018; Zhu et al., 2018) with some modifications. Hippocampal regions (DG, CA1, CA2-4, Subiculum) and adjacent entorhinal cortex were dissected from three frozen adult human brains (table S). In order to avoid experimental bias and evenly dissociate the tissue for cell nuclei isolation, whole tissue was finely pulverized to powder in liquid nitrogen with mortar and pestle (Coorstek \#60316, \#60317). All buffers were ice cold and all reagents used for consequent nuclear isolation were molecular biology grade unless stated otherwise. 5 - $10 \mathrm{mg}$ of pulverized tissue was added into $5 \mathrm{ml}$ of ice-cold lysis buffer consisting of $320 \mathrm{mM}$ sucrose (Sigma \#S0389), $5 \mathrm{mM} \mathrm{CaCl2} \mathrm{(Sigma} \mathrm{\# 21115),} 3 \mathrm{mM}$ $\mathrm{Mg}$ (Ace)2 (Sigma \#63052), 10mM Tris-HCl (pH 8) (AmericanBio \#AB14043), protease inhibitors w/o EDTA (Roche \#11836170001), 0.1 mM EDTA (AmericanBio \#AB00502), RNAse inhibitor (80U/ml) (Roche \#03335402001), 1mM DTT (Sigma \#43186), and 0.1\% TX-100 (v/v) (Sigma\#T8787). DTT, RNAse Protector, protease inhibitors, and TX-100 were added immediately before use. The suspension was transferred to Dounce tissue grinder $(15 \mathrm{ml}$ volume, Wheaton \#357544; autoclaved, RNAse free, ice-cold) and homogenized with loose and tight pestles, 30 cycles each, with constant pressure and without introduction of air. The homogenate was strained through 40 um tube top cell strainer (Corning \#352340) which was pre-wetted with $1 \mathrm{ml}$ wash buffer: (250 mM sucrose (Sigma \#S0389), 25 mM KCl (Sigma \#60142), 5mM MgCl2 (Sigma \#M1028), 20mM Tris-HCl (pH 7.5) (AmericanBio \#AB14043; Sigma \#T2413), protease inhibitors w/o EDTA (Roche \#11836170001), RNAse inhibitor (80U/ml) (Roche \#03335402001), 
900

901

902

903

904

905

906

907

908

909

910

911

912

913

914

915

916

917

918

919

920

921

922

1mM DTT (Sigma \#43186)). Additional $4 \mathrm{ml}$ of wash buffer was added to wash the strainer. Final $10 \mathrm{ml}$ of solution was mixed with $10 \mathrm{ml}$ of 50\% Optiprep (Axis-Shield\# 1114542) solution (50\% iodixanol (v/v), $250 \mathrm{mM}$ sucrose (Sigma \#S0389), 25 mM KCl (Sigma \#60142), 5mM MgCl2 (Sigma \#M1028), 20mM Tris-HCl (pH 7.5) (AmericanBio \#AB14043; Sigma \#T2413), protease inhibitors w/o EDTA (Roche \#11836170001), RNAse inhibitor (80U/ml) (Roche \#03335402001), 1mM DTT (Sigma \#43186)) by inverting the tube 10x and carefully pipetted into 2 centrifuge tubes (Corning \#430791). The tubes were centrifuged at $1000 \mathrm{~g}$, for $30 \mathrm{~min}$ at $4{ }^{\circ} \mathrm{C}$ on centrifuge (Eppendorf \#5804R) and rotor (Eppendorf \#S-4-72). Upon end of centrifugation, the supernatant was carefully and completely removed and total of $5 \mathrm{ml}$ of resuspension buffer $(250 \mathrm{mM}$ sucrose (Sigma \#S0389), 25 mM KCl (Sigma \#60142), 5mM MgCl2 (Sigma \#M1028), 20mM Tris-HCl (pH 7.5) (AmericanBio \#AB14043; Sigma \#T2413), protease inhibitors w/o EDTA (Roche \#11836170001), RNAse inhibitor (80U/ml) (Roche \#03335402001), 1mM DTT (Sigma \#43186)) was added carefully on the pellets in tubes and centrifuged at $1000 \mathrm{~g}$, for 10 min at $4{ }^{\circ} \mathrm{C}$ on the same centrifuge and rotor. Supernatants were then carefully and completely removed, pellets were gently dissolved by adding $100 \mathrm{ul}$ of resuspension buffer (see above) and pipetting 30x with $1 \mathrm{ml}$ pipette tip, pooled and filtered through 35 um tube top cell strainer (Corning \#352340). Finally, nuclei were counted on hemocytometer and diluted to 1 million/ml with sample-run buffer: $0.1 \%$ BSA (Gemini Bio-Products \#700-106P), RNAse inhibitor (80U/ml) (Roche\#03335402001), 1mM DTT (Sigma \#43186) in DPBS (Gibco \#14190).

\section{Single nucleus microfluidic capture and cDNA synthesis}

The nuclei samples were placed on ice and taken to Yale Center for Genome Analysis core facility and processed within 15 minutes for single nucleus RNA sequencing with targeted nuclei recovery 
923 of 10000 nuclei, respectively, on microfluidic Chromium System (10x Genomics) by following

924 the manufacturer's protocol (10x Genomics, CG000183_Rev_A), with Chromium Single Cell 3'

925 GEM, Library \& Gel Bead Kit v3, (10x Genomics \#PN-1000075) and Chromium Single Cell B

926 Chip Kit (10x Genomics \#PN-1000074), Chromium i7 Multiplex Kit (10x Genomics \#PN-

927 120262) on Chromium Controller (10x Genomics). Due to limitations imposed by source RNA

928 quantity, cDNA from nuclei was amplified for 14 cycles.

929

930 Single nucleus RNA-seq library preparation

931 Post cDNA amplification cleanup and construction of sample-indexed libraries and their

932 amplification followed manufacturer's directions (10x Genomics, CG000183_Rev_A), with the

933 amplification step directly dependent on the quantity of input cDNA.

934

935 Sequencing of libraries

936 In order to reach sequencing depth of 20000 raw reads per nucleus, single nucleus libraries were

937 run using paired end sequencing with single indexing on the HiSeq 4000 platform (Illumina) by

938 following manufacturer's instructions (Illumina; 10x Genomics, CG000183_Rev_A). To avoid

939 lane bias, multiple uniquely indexed samples were mixed and distributed over several lanes. RNA-

940 seq data is deposited at http://psychencode.org and NCBI dbGAP Accession phs000755.v2.p1.

941

942 Quantification and Statistical Analysis

943 Single nuclei expression quantification and quality control

944 We quantified the expression levels of genes in each potential nucleus represented by a cellular

945 barcode using the 10X Genomics CellRanger pipeline (version 3.0.2). Reads were mapped to 
human reference genome GRCh38 (Ensembl release 98) and quantified in units of Unique Molecular Identifiers (UMIs) based on the combined exon-intron human annotation. We took advantage of the enhanced cell-calling methodology in CellRanger to distinguish true cells from damaged or empty droplets. Specifically, RNA content distribution of each barcode was compared to the background concentration which was generalized from extremely low RNA-containing barcodes, and was subsequently classified as damaged if comparable profiles were seen. To further rule out low-quality cells, we excluded nuclei with mitochondrial content greater than $10 \%$. This loose criterion was set as we aimed to incorporate certain cell types into analyses such as endothelial cells which were shown to be prone to high mitochondrial content (Velmeshev et al., 2019). Additional filtering procedure was performed after clustering and low-dimensional embedding (see below) to eliminate small cell clusters collectively displaying elevated mitochondrial and ribosomal gene expression and showing no signals of reasonable cell types.

\section{Normalization, dimensionality reduction and clustering}

We normalized the raw UMI counts into log-transformed Transcripts Per Million (TPM) using the 'NormalizeData' function in the R package Seurat (scaling factor equals to 10,000) (version 3.1.0) (Butler et al., 2018). To position all nuclei in a two-dimensional representation reflecting their transcriptomic similarities (Fig. 1B-1D), the top 2,000 highly variable genes were obtained by the Seurat function 'FindVariableFeatures' with the default variance stabilizing process for each of the three human individuals. We further integrated nuclei from the three humans on the basis of their anchor features summarized from each individual via the function 'IntegrateData' and embedded ensuing nuclei in the Uniform Manifold Approximation and Projection (UMAP) plot using the top 30 principal components (PCs) ('RunPCA' function in Seurat followed by the 
function 'RunUMAP'). To cluster nuclei according to their nearest transcriptomic neighbors, we searched for shared nearest neighbors (SNN) in the PCA space with the neighbor number being 20 and optimized the graph modularity using the Seurat function 'FindClusters'. In general, we performed an iterative removal-clustering approach to remove nuclei with high mitochondrial or ribosomal contents and without clear cluster-related markers followed by re-clustering of the remaining nuclei. Moreover, cells co-expressing multiple cell-type specific marker genes were manually marked as doublets and excluded from the downstream analytical flow. Lastly, we reembedded cell types of interest (i.e., excitatory neurons, inhibitory neurons and non-neuronal cells) in the UMAP space and re-clustered them using the same procedure as mentioned above, as this would offer finer details into the cell types we sought to probe into.

\section{Global across-dataset comparison}

We performed global comparisons with the human MTG (Hodge et al., 2019) and dlPFC single nucleus datasets (Li et al., 2018) to investigate the similarities and distinctions among them. We processed the MTG and dIPFC data using the same procedure except that for MTG, the scaling factor during data normalization was set to $1,000,000$ to mitigate the bias caused by magnitude differences. For each of the highly variable genes detected in both our data and MTG/dlPFC data, we averaged the TPMs across each subtype within both data and transformed them in the logarithmic space. Spearman correlation coefficients were calculated across these subtypes to further avoid the across-dataset batches and the resulting linkages were exhibited in gradient heat maps (Fig. S1E, S1F).

\section{Tree construction}


992 To explore the taxonomic relationships among all cell subtypes, we constructed a hierarchical tree

993 by first averaging the gene expression levels across cells of the same subtype. The derived expression was standardized to mean of zero and variance of one within each subtype across the anchor genes selected in the previous integration step. Following this step, we calculated the Euclidean distances between pairwise subtypes, and clustered these subtypes in a structured tree (Fig. 1E) by the 'hclust' function in R with the method set to 'ward.D2'.

\section{Classification of cell subtypes}

We grouped cell clusters with strong signals of SLC17A7 expression into excitatory neurons. of $P R O X 1$. Mossy cells were described by the principal origin from dentate gyrus and exclusive expression of $A D C Y A P 1$. We subsequently classified granule cells into three subtypes characterized by the high expression of SGCZ, PDLIM5 and EGR1, respectively. Excitatory neurons from CA fields were arranged mainly according to subfields: CA3 pyramidal neurons (coexpression of CFAP299 and SYN3), CA2 pyramidal neurons (co-expression of CFAP299 and

1011 we categorized them into three subtypes: one distal (away from CA1) $(F N 1+$ ) subtype and two 1012 proximal ones $(\mathrm{ROBO}+\mathrm{H})$. Of note, the spatial registrations of $\mathrm{CA}$ and subiculum cell subtypes

1013 were achieved on the basis of previous transcriptomic studies of hippocampal pyramidal neurons

1014 (Cembrowski et al., 2016a; Cembrowski et al., 2016b; Cembrowski et al., 2018). With regards to 
1015 entorhinal excitatory neurons, we classified them by two means. First, we aligned them with 1016 excitatory neurons from single nucleus data of human MTG using the same procedure as described

1017 above. Second, we examined the subtype-specific marker genes in both our excitatory neurons and 1018 related literature reports. Specifically, two layer 2 subtypes were classified as $R E L N+$ and one as $1019 C A L B 1+($ Witter et al., 2017). Other upper-layer subtypes were depicted based on marker gene 1020 expression of LAMA3, PDGFD, IL1RAPL2, and PCP4 (Ramsden et al., 2015; Tang et al., 2015; 1021 Ohara et al., 2018). The middle-to-deep layer subtypes were delineated by the specific gene 1022 expression of RORB, THEMIS, ADRA1A, and TLE4. Inhibitory neuron clusters were first classified to major groups based on the expression of three canonical function markers $(P V A L B, S S T, V I P)$ as well as $L A M P 5$, a marker mostly representing a group of neurogliaform inhibitory neurons and recently being adopted as a major inhibitory 1027 neuron marker (Tasic et al., 2018; Hodge et al., 2019). For a cluster expressing two markers 1028 simultaneously (eg. InN LAMP5 NMBR cluster expresses both SST and LAMP5), it was assigned 1029 to the same major group of the neighboring cluster in the hierarchical tree. Additionally, we used LHX6 (a medial ganglionic eminence marker) and NR2F2 (a caudal ganglionic eminence marker) to classify the rest of the inhibitory neuron clusters which do not express these markers. We also

1032 identified an inhibitory neuron cluster with no evident markers and showing high mitochondria 1033 counts, indicative of low-quality cells, which accordingly was termed as "InN unknown”. Finally, 1034 each inhibitory neuron cluster was named after the combination of major group marker (eg. SST, 1035 VIP) and one top exclusive cluster marker (eg. ANO2). Apart from these inhibitory neuron 1036 clusters, we also identified a neuron cluster co-clustered with inhibitory neurons showing strong 1037 signals of RELN, NDNF, highly indicative of Cajal Retzius cells. 
1042 (oligodendrocyte precursor cells, OPCs), GPR17 (committed oligodendrocyte precursor cells,

1043 COPs), and MOBP (oligodendrocytes), as in Fig. S2H. We additionally grouped OPCs and 1044 oligodendrocytes into specific subtypes according to the high expression of specific genes: EGRI 1045 and GRIA4 for OPCs; CPXM2, SLC5A11, LINC01098 and LAMA2 for oligodendrocytes. For astrocyte subtype specification, we classified them by the laminar distribution: $G F A P+$ ones located in deep layers and CHRDL1+ ones in upper layers (Fig. S2G) (Lanjakornsiripan et al., 2018). Regarding immune cells, we used marker genes C1QB, F13A1, LYZ and SKAPI to deconstruct them into microglia, macrophages, myeloid cells and T cells, respectively (Fig. S2I). Microglia were further subdivided via specific gene expression of $P 2 R Y 12$ and $C D 83$. In terms of endothelial cells $(D K K 2+)$, endothelial cells $(C L D N 5+$ and $V W F+)$, pericytes $(C L D N 5+$ and $A B C C 9+)$, venous smooth muscle cells $(A B C C 9+$ and $P 2 R Y 14+)$, arterial smooth muscle cells $(A C T A 2+$ and $T A G L N+)$ and vascular and leptomeningeal cells $(C O L 1 A 2+$ and $C O L 1 A 1+)($ Fig. S2J) (Vanlandewijck et al., 2018).

\section{Cell subtype comparisons among HIP, EC, MTG and dIPFC}

1058 To explore the transcriptomic divergence across HIP, EC, MTG and dlPFC for all cell subtypes, 
1061 first determined the marker genes of each subtype using the 'FindAllMarkers' function in Seurat.

1062 Subsequently, we generated a similarity matrix representing the overlap between marker genes of

1063 pairwise subtypes across all regions, followed by the visualization of this matrix in the form of a

1064

1065

1066

1067

1068

1069

1070

1071

1072

1073

1074

1075

1076

1077

1078

1079

1080

1081

1082

1083

network via the R package 'igraph’ through the force-directed graphopt algorithm (Fig. 2A-F).

Especially, for excitatory neuron types we displayed their connections in a between-region manner (HIP and EC, EC and MTG, and MTG and dlPFC). To further examine the cell subtype connections across different regions, in each brain region we focused on marker genes detected in at least one subtype and assessed their expression across all subtypes of remaining brain regions visualized in heat maps (Fig. S3A). Additionally, given the upper- and deep-layer marker genes identified in MTG, we calculated the percentages of genes in each subtype of each region where expression was greater than the expression constraint of $75 \%$ quantile across all expression values. Furthermore, we evaluated the expression of marker genes from intratelencephalic/intracerebral (IT) neurons and non-IT neurons of MTG in all subtypes of the four regions through first averaging the expression of each gene across cells of the same subtype and then displaying the median values across IT markers/Non-IT markers in scatter plots (Fig. S3C).

\section{Exclusive markers of cluster InN SST ADAMTS12}

To find hippocampus-specific transcriptome features in the cluster InN SST ADAMTS12, we first sought to confirm the enrichment of this cluster in hippocampus by integrating inhibitory neurons from across the hippocampal-entorhinal system with those from MTG and dlPFC using the 'fastMNN' function from the batchelor R package (Fig. 3C) (Haghverdi et al., 2018). Following this confirmation, we identified a set of markers exclusively expressed in this cluster as compared to other interneuron clusters in hippocampus and SST-expressing interneuron clusters in MTG or 
1084 dlPFC. To do so, we first calculated a specificity score for each gene in each cluster to assess the

1085

1086

1087

1088

1089

1090

1091

1092

1093

1094

1095

1096

1097

1098

1099

1100

1101

1102

1103

1104

1105

1106

specificity of gene expression in each cluster (Li et al., 2018). For cluster InN SST ADAMTS12,

we then selected those genes with a specificity score in the $99 \%$ quantile and that showed a 1.5

fold or greater difference between this specificity score and the maximum specificity score of that gene in any other cluster. To further refine this list of genes exhibiting highly selective expression

in In SST ADAMTS12, we next removed from this list those genes that were also highly specific

(maximum specificity score in the $99^{\text {th }}$ quantile) for any population of $S S T$-expressing neurons in MTG or dlPFC. The expression of these markers in each cell was then generalized as a single AUROC score calculated via AUCell package (Aibar et al., 2017).

\section{Expression of DCX and proliferation markers in HIP and EC}

To check the expression of $D C X$ in the cell types of HIP and EC, we interrogated $D C X$ expression in all excitatory or inhibitory neurons and next in all DG or EC cell types, all of which were reembedded in the UMAP space (Fig. 4A-D). During visualization, the expression threshold (i.e., log-transformed TPM) was set to one to highlight the apparent expression of $D C X$ in corresponding cell types. Quantitatively, we evaluated the percentage of DG inhibitory neurons or GC expressing DCX under different thresholds in units of UMIs (1, 2 and 3). We further examined the cells expressing NES, MKI67 or DCX, the three markers labeling neural stem cells (NSC), neural progenitor cells (NPC), and migrating and immature granule cells (IM) respectively, in DG and EC. These cells were uncovered in each subtype of DG or EC, and the marker genes over the granule cell maturation were checked specifically for those cells (Fig. 4E, 4F and SFB, S4C).

To test whether DCX-expressing cells showed enriched expression of NEUROD1, NEUROD2, SOX11, CALB2, FXYD7, STC1, NCAM1 and PROX1, we compared, in each cluster, 
1107 the proportion of $D C X$-expressing and $D C X$-negative cells also expressing each of those markers

1108 by means of a Fisher Exact's test (Fig. S4A). P-values were adjusted using Bonferroni correction.

\section{Analysis of bulk tissue transcriptomic datasets}

1112 Gene expression analysis was performed on an exon array (Kang et al., 2011) and PsychENCODE

1113 RNA-seq datasets (Li et al., 2018). Gene expression values from exon array were used to rank

1114 protein-coding genes based on a region-specific upward or downward temporal trajectory. Time

1115 periods 3-15 were collapsed into three time groups: prenatal (periods 3-7), early postnatal (periods

1116 8-12), and adult (periods 13-15). We used limma (Smyth, 2005) to run a regression that included

1117 the time group and brain region, as well as the region-group interactions, as factors. To select genes

1118 with an increase in expression across time groups in a single region, genes were ranked by their

1119 region-group interaction coefficient. We filtered to genes for which the time group coefficient was

1120 above an arbitrary cutoff of -0.05 to remove cases where the high region-group interaction simply

1121 offsets an overall negative decrease in expression across the time groups. We also filtered out

1122 genes where more than one region-group interaction coefficient was above 0.01 to restrict the

1123 ranking to increases in expression that are unique to a single brain region. To identify genes where

1124 expression decreases across time groups in a region-specific manner, similar criteria were used,

1125 reversing the sign of the cutoff values and the direction of the comparisons. Gene expression values

1126 from BrainSpan RNA-seq dataset were used to compare METTL7B expression within multiple

1127 neocortical regions during adulthood (time periods 13-15). Statistical analysis was performed

1128 using one-way ANOVA. 


\section{Generation of knockout mice and tissue processing}

1131 All experiments with mice were performed in accordance with a protocol approved by Yale

1132 University's Committee on Animal Research. Targeted embryonic stem (ES) cells

1133 (Mettl7b $\left.\mathrm{b}^{\mathrm{tm} 1(\mathrm{KOMP}) \mathrm{Vlcg}}\right)$ were obtained from Knockout Mouse Project (KOMP) repository. Chimeric

1134 mice were generated by blastocyst injection of ES cells at Yale Genome Editing Center (YGEC).

1135 Mice were bred for germline transmission to generate gene knockout mice. Genotyping was

1136 performed using the TUF/TUR primer set (145 bp) for the wild-type allele and the NeoFwd/SD

1137 primer set $(351 \mathrm{bp})$ for the Mettl7b deletion allele.

1138 Both wild type and Mettl7b mutant mice were reared in group housing in a $12 \mathrm{~h}$ light:12h

1139 dark cycle and provided food and water ad libitum with veterinary care provided by Yale Animal

1140 Resource Center. Only mice in good, healthy condition, as approved by Yale Animal Resource

1141 Center, were used for breeding and experimentation. Multiple breeding pairs were maintained and

1142 siblings were never mated to increase genetic diversity, and prevent unintended selection for

1143 features that could affect results. Both sexes were used and randomly assigned for all experiments.

1144 Adult mice were anesthetized and intracardially perfused with ice-cold PBS and 4\% PFA. All

1145 mouse brain tissue specimens were fixed by immersion in $4 \%$ PFA overnight at $4{ }^{\circ} \mathrm{C}$ and sectioned

1146 at $50 \mu \mathrm{m}$ using a vibratome (Leica).

$1148 \quad \underline{\text { In situ hybridization }}$

1149 Human brain tissue samples were fixed in $4 \%$ PFA overnight at $4{ }^{\circ} \mathrm{C}$ and sectioned at $30 \mu \mathrm{m}$ using

1150 a Leica VT1000 S vibratome. The RNA probes complementary to human METTL7B cDNA

1151 (NM_152637.2) were labeled with digoxigenin-UTP (Roche). After acetylation, sections were

1152 hybridized with the probes at $63^{\circ} \mathrm{C}$ for 16 hours. Following hybridization, the riboprobes were 
1153 immunolabeled with anti-digoxigenin-AP conjugate and the signal was developed with NBT/BCIP

1154 overnight in dark.

1155

1156

Immunolabeling and histology

1157 For METTL7B immunohistochemistry, tissue sections were processed using ImmPRES Excel 1158 Amplified HRP Polymer Staining Kit (Anti-Rabbit IgG, MP-7601-15, Vector Laboratories) per

1159 manufacturer's protocol. For mouse $\alpha$ - $\beta$-galactosidase (lacZ) stain, tissue sections were blocked 1160 with blocking solution (5\% normal donkey serum, $1 \%$ BSA, $0.1 \%$ glycine, $0.1 \%$ lysine, and $0.3 \%$

1161 Triton X-100 in PBS) for 1 hour and incubated with primary antibodies and biotinylated secondary

1162 antibodies. The signal was amplified with Vectastain ABC-AP kit (AK-5000, Vector Labs) and

1163 developed with Vector Blue AP kit (SL-5300, Vector Labs) per manufacturer's protocol. DCX

1164 and GAD1 immunohistochemistry was performed with anti-DCX antibodies raised in guinea pig

1165 (EMD Millipore AB2253; 1:1000) and anti-GAD1 antibody raised in goat (R\&D AF2086; 1:200)

1166 in 3\% normal donkey serum, $0.25 \%$ Triton X-100 in PBS). Antigen retrieval (20 mins in citrate

1167 buffer $\mathrm{pH} 6$ at 95C) was required for optimal results. Antibody detection was achieved with

1168 biotinylated secondary antibody and Streptavidin conjugated (Jackson Immunoresearch) for DCX

1169 and anti-goat secondary antibodies (Jackson Immunoresearch) for GAD1. DAPI was used for

1170 nuclear staining. All histology samples were imaged on Aperio ScanScope system or imaged on a

1171 Zeiss LSM 510 confocal microscope. Cell culture samples were fixed with ice-cold 4\%

1172 paraformaldehyde (PFA) for 10 minutes at room temperature, blocked for 30 minutes at RT with

1173 blocking solution (5\% normal donkey serum, 1\% BSA, $0.1 \%$ glycine, $0.1 \%$ lysine, and $0.3 \%$

1174 saponin in PBS), incubated with primary and appropriate Alexa Flour-conjugated secondary

1175 antibodies, and imaged on Zeiss LSM 510 confocal microscope. 
$1177 \quad$ Plasmids

1178 For expression of METTL7B, full length cDNA (NM_152637.2) was inserted into pCAGIG (a gift

1179 from Connie Cepko, Addgene \#11159) (Matsuda and Cepko, 2004). For lentiviral generation,

1180 pFUGW (a gift from David Baltimore, Addgene \#14883) (Lois et al., 2002) was digested with

1181 PacI, 3' overhangs removed with Klenow (NEB) to form blunt ends, and additionally digested

1182 with BsrGI to release hUBC promoter and EGFP. The CAG-IRES-EGFP was removed from

1183 pCAGIG and ligated into pFUGW. For protein pulldown experiments, BirA-HA and HaloTag

1184 constructs were PCR-amplified from pcDNA3.1-MCS-BirA(R118G)-HA (a gift from Kyle Roux,

1185 Addgene \#36047) (Roux et al., 2012) and pHTC-CMVneo-HaloTag (G7711, Promega),

1186 respectively, and ligated into pFUGW-CAG. For overexpression of wild type APP, full length

1187 APP (NM_201414.2) and mCherry cDNA were PCR amplified and ligated into pFUGW-CAG in

1188 place of IRES-EGFP. For doxycycline inducible expression of METTL7B, cDNA fragments

1189 [Ampicilin resistance, high-copy-number Origin of replication, SV40 poly(A), and IRES-EGFP

1190 from pCAGIG; rTetR and tight TRE promoter from pCW57.1 (a gift from David Root, Addgene

1191 \#41393); hPKG promoter (M60581.1); METTL7B (NM_152637.2); bGH poly(A) from pFUGW]

1192 were PCR amplified, ligated, and circularized (pDTET-METTL7B).

$1194 \quad$ Lentiviral purification and generation of stable cell lines

1195 Ten 15-cm dishes of sub-confluent Lenti-X 293T cells (Clontech) were used for each purification. 1196 pFUGW-CAG specific plasmids (BirA, METTL7B-BirA, HaloTag, METTL7B-HaloTag) along

1197 with pMD2.G, pRSVrev and pMDLg/pRRE (a gift from Didier Trono, Addgene \#12259, \#12253, 1198 \#12251) (Dull et al., 1998) were transfected at 1:1:1:1 molar ratio using PolyJet (SignaGen). Cell 
1199 culture media containing lentiviral particles (LVP) was collected at 48- and 60-hours post-

1200 transfection and filtered through $0.2 \mu \mathrm{m}$ filter to remove cellular debris. Filtered supernatants were

1201 centrifuged at 100,000g for 2 hours. One milliliter of PBS was laid over LVP pellet and left

1202 overnight at $4{ }^{\circ} \mathrm{C}$. Next day, resuspended pellets were centrifuged through $30 \%$ sucrose gradient

1203 to further purify the virus. Lentiviral titers were determined by transducing Lenti-X 293T cells and

1204 calculating titer from FACS data between $1-10 \%$ infection rate using formula: Titer $(\mathrm{IU} / \mathrm{ml})=(\#$

1205 cells seeded $\mathrm{x}$ dilution factor $\mathrm{x} \%$ GFP-positive cells) / (volume of virus solution added).

1206

1207 For pulldown experiments, 50,000 ReNcell CX (EMD Millipore) cells were plated on a laminin 1208 coated 24-well plate in triplicate wells. Cells were transduced with lentiviral particles at MOI of

120910 in a $150 \mu \mathrm{L}$ of cell culture media supplemented with $10 \mu \mathrm{g} / \mathrm{mL}$ of protamine sulfate 1210 (\#02194729, MP Biomedicals) and saved as ReN-CAG-BirA, ReN-CAG-METTL7B-BirA, ReN-

1211 CAG-HaloTag, and ReN-CAG-METTL7B-HaloTag stable cell lines.

1212

1213 For $A P P$ overexpression, N2a cells were transduced at MOI of 40. After propagation, eight million

1214 cells were sorted at Yale Flow Cytometry Facility, Yale University, on a BD SORP FACSAria 2

1215 cell sorter (Special Order Research Product) using the 100- $\mu \mathrm{m}$ nozzle and a sheath pressure of 20

1216 p.s.i. BD FACSDiva Software was used to acquire and analyze samples. Gates were set to remove

1217 cell doublets and particle debris. Cell selection was based on mCherry signal intensity and top one

1218 percent expressing cells were saved as the N2a-APP cell line. 
1221 For BioID and HaloTag experiments, two million cells (ReN-CAG-BirA, ReN-CAG-METTL7B-

1222 Bira, ReN-CAG-HaloTag, ReN-CAG-METTL7B-HaloTag) were plated on four laminin coated

1223 10-cm dishes. BioID pulldown was performed per protocol (Roux et al., 2013). At near confluency,

1224 cell culture media was supplemented with $50 \mu \mathrm{M}$ biotin (B4639, Sigma-Aldrich). The next day,

1225 cells were rinsed twice with PBS, detached with Accutase (Millipore) for 10 minutes at $37^{\circ} \mathrm{C}$,

1226 centrifuged at $200 \mathrm{~g}$ for 3 minutes, rinsed with PBS, and centrifuged again. Bead-protein

1227 conjugates were resuspended in $50 \mathrm{mM}$ ammonium bicarbonate. HaloTag pulldown was

1228 performed per manufacturer's protocol (G6500, Promega). Proteins were eluted by resuspending

1229 HaloTag resin in $50 \mu \mathrm{L}$ of $8 \mathrm{M}$ urea prepared in $50 \mathrm{mM}$ ammonium bicarbonate and shaking for

123030 minutes at room temperature. Ten percent fractions of BioID and HaloTag eluates were saved

1231 for immunoblot and silver stain analysis.

1232

$\underline{\text { Mass spectrometry and proteomic data analysis }}$

1234 BioID and HaloTag tryptic digestion was performed using the optimized method from the original

1235 published method(Kim et al., 2014). Proteins were reduced by adding $2 \mu \mathrm{l}$ of $0.5 \mathrm{M}$ Tris(2-

1236 carboxyethyl)phosphine (TCEP) at $30^{\circ} \mathrm{C}$ for $60 \mathrm{~min}$. The reaction was cooled to room temperature

1237 (RT) and proteins were alkylated in the dark for $30 \mathrm{~min}$ by adding $4 \mu \mathrm{l}$ of $0.5 \mathrm{M}$ Iodoacetamide.

1238 Sample volume was adjusted by adding $350 \mu \mathrm{l}$ of $50 \mathrm{mM}$ Ammonium Bicarbonate to dilute the

$12398 \mathrm{M}$ urea to $1 \mathrm{M}$ before trypsin digestion. Mass spectrometry grade trypsin (Promega) was added

1240 for overnight digestion at $30^{\circ} \mathrm{C}$ using Eppendorf Thermomixer at $700 \mathrm{rpm}$. Formic acid was added

1241 to the peptide solution (to $2 \%$ ), followed by desalting by C18 TopTip (TT10C18.96, PolyLC) and

1242 finally dried on a SpeedVac. Tryptic peptides were resuspended in $100 \mu 1$ of $2 \%$ Acetonitrile in

$1243 \quad 0.1 \%$ formic acid. Ten microliters of total tryptic peptides were used in triplicate runs for the 1D 
1246 spectrometer (all from Thermo Fisher Scientific). A 230-min gradient consisting of 5-16\%B

1247 (100\% acetonitrile) in $140 \mathrm{~min}, 16-28 \%$ in $70 \mathrm{~min}, 28-38 \%$ in $10 \mathrm{~min}, 38-85 \%$ in $10 \mathrm{~min}$ was used

1248 to separate the peptides. The total LC time was 250 min. The Q Exactive Plus was set to scan 1249 precursors at 70,000 resolution followed by data-dependent MS/MS at 17,500 resolution of the top

125012 precursors.

1251

1253 combined and submitted to Sorcerer Enterprise v.3.5 release (Sage-N Research Inc.) with

1254 SEQUEST algorithm as the search program for peptide/protein identification. SEQUEST was set

1255 up to search the target-decoy UniProt Human Reviewed (v. March 2015) protein fasta database

1256 using trypsin for the enzyme and with the allowance of up to 2 missed cleavages, semi tryptic

1257 search, fixed modification of $57 \mathrm{Da}$ for cysteines to account for carboxyamidomethylation and

1258 precursor mass tolerance of $50 \mathrm{ppm}$. Differential search included $226 \mathrm{Da}$ on lysine for biotinylation

1259 (BioID samples), 16 Da for methionine oxidation, and 14, 28 and 42 Da on lysine for mono-, di-

1260 and tri- methylayion. The search results were viewed, sorted, filtered, and statically analyzed by

1261 using comprehensive proteomics data analysis software, Peptide/Protein prophet v.4.02 (ISB). The

1262 minimum trans-proteomic pipeline (TPP) probability score for proteins was set to 0.9 to assure

1263 very low error (less than FDR 2\%) with good sensitivity. The differential spectral count analysis

1264 was done by QTools, an open source SBP in-house developed tool for automated differential

1265 peptide/protein spectral count analysis(Brill et al., 2009) and the protein prophet peptide report

1266 was utilized to report biotinylated peptides. The LC-MS/MS raw data were also submitted to 
1267 Integrated Proteomics Pipelines (IP2) Version IP2 v.3 (Integrated Proteomics Applications, Inc.)

1268 with ProLucid algorithm as the search program (Xu et al., 2006) for peptide/protein identification.

1269 ProLucid search parameters were set up to search the UniProt Human Reviewed (v. March 2015)

1270 protein fasta database including reversed protein sequences using trypsin for enzyme with the

1271 allowance of up to 2 missed cleavages, semi tryptic search, fixed modification of 57 Da for

1272 cysteines to account for carboxyamidomethylation and precursor mass tolerance of $50 \mathrm{ppm}$.

1273 Differential search included 226 Da on lysine for biotinylation (for BioID samples), 16 Da for

1274 methionine oxidation, and 14, 28 and $42 \mathrm{Da}$ on lysine for mono-, di- and tri- methylayion. The

1275 search results were viewed, sorted, filtered, and statically analyzed by using DTASelect for

1276 proteins to have protein FDR rate of less than 2.5\% (Tabb et al., 2002). Differential label-free

1277 proteomics data analysis was done by IP2-Census, Protein Identification STAT COMPARE (Park

1278 et al., 2008) using two technical replicates. This result was a label-free quantification analysis, of

1279 duplicate technical data for each sample; using spectral count analysis with t-test and Gene

1280 Ontology analysis (Robinson et al., 2004).

Identification of true pulldown proteins based on mass spectrometry spectral counting data: We

1283 discriminated true prey-bait interactions from false interactions in the Halotag and BioID

1284 pulldowns by using Significance Analysis of INTeractome (SAINT) method (Choi et al., 2011;

1285 Teo et al., 2014). Briefly, the SAINT method utilizes MS/MS spectral counting data and models

1286 true and false prey-bait interactions as separate Poisson distributions to obtain the probability of

1287 a true protein-protein interaction based on Bayesian statistical inference. The estimated probability

1288 provides a quantitative measure of the confidence of prey-bait interactions such that false

1289 interactions can be filtered out in a statistically-controlled manner. Upon applying the SAINT 
method to MS/MS spectral count data available from each pulldown experiment system, we identified 275 (out of 3 cases and 3 controls) and 1795 ( 3 cases and 3 controls) proteins as true METTL7B interactors from Halotag and BioID pulldowns, respectively, at Bayesian False Discovery Rate (BFDR) of 5\%. interactors, we performed fold-enrichment test for major subcellular compartments cataloged in

1297 the Human Protein Atlas database (Uhlen et al., 2015) and mammalian lipid droplet proteomes 1298 (Hodges and Wu, 2010). Human Protein Atlas provides genome-wide analysis of major subcellular 1299 localization information of human proteins based on immunofluorescent stained cells. It consists 1300 of 20 main subcellular compartments and 10,003 proteins (www.proteinatlas.org). To make the 1301 fold-enrichment test comparable across Human Protein Atlas and the mammalian lipid droplet 1302 proteome datasets, we merged the mammalian lipid droplet protein list to Human Protein Atlas 1303 dataset as a separate subcellular localization category and used the entire Human Protein Atlas 1304 subcellular localization records uniformly as a null (background) set. We found that $73.8 \%$ $1305(203 / 275)$ and $77.7 \%(1384 / 1795)$ of true METTL7B interactors from HaloTag and BioID 1306 pulldown experiments had matching HGNC gene symbols in Human Protein Atlas. Of the 152 1307 mammalian cytoplasmic lipid proteins(Hodges and $\mathrm{Wu}, 2010), 80$ proteins had matching HGNC 1308 gene symbols in the Human Protein Atlas. Twenty-three (HaloTag) and 37 (BioID) true 1309 METTL7B interactors were identified to be among 80 lipid droplet proteins in the Human Protein 1310 Atlas database. 
1313 (comparison with the BioGRID protein-protein interaction database): We evaluated the

1314 performance of SAINT method by benchmarking the true METTL7B interactors against non-

1315 redundant physical BioGRID protein-protein interaction network (Stark et al., 2006). We

1316 computed the significance of interactions between proteins from the true METTL7B interactor set

1317 and the rest of the proteins (background set) in the protein-protein interaction (PPI) network by

1318 using binomial proportions test Z-score as follows (Abul-Husn et al., 2009):

1319

$$
\mathrm{Z}=\frac{p_{1} / N_{1}-p_{2} / N_{2}}{\sqrt{\mathrm{p}(1-\mathrm{p}) \cdot\left(1 / N_{1}+1 / N_{2}\right)}}
$$

1321 where

1322

$p_{1}$ : number of true METTL7B interactors among the adjacent PPI network neighbors of a given

1324 protein,

$1325 p_{2}$ : number of all the adjacent PPI network neighbors of a given protein,

$1326 N_{1}$ : number of the true METTL7B interactors present in the PPI network,

$1327 \quad N_{2}:$ number of the all PPI network proteins, and

$1328 p=(p 1+p 2) /(N 1+N 2)$.

1330 The Z-score thus provides an approximate quantitative measure of how significantly a given 1331 protein in the PPI network interacts with the true METTL7B interactors in the immediate 1332 neighborhood of the protein-protein interaction network compared to the background proteins in 1333 the protein-protein interaction network. We found that the true METTL7B interactors tend to 1334 interact much more significantly to each other than to the rest of proteins in the protein-protein 
1335 interaction network (Wilcoxon rank sum test $\mathrm{p}$-value $<2 \mathrm{e}-16$, data not shown). This indicates that

1336 the true METTL7B interactors are significantly clustered and proximal to each other in the protein-

1337 protein interaction network as expected.

1340 the true METTL7B interactors, we performed KEGG pathway enrichment analysis using the

1341 binomial proportion test Z-scores as weights of proteins in a given pathway. The rationale for using

1342 such a weight is that the proteins interacting significantly more with true METTL7B interactors

1343 play proportionally important role in specifying the biological context represented by the true

1344 METTL7B interactors. To this end, we assigned biological context specificity scores to all KEGG

1345 pathways in a similar manner used in (Ideker et al., 2002) as follows:

1347 First, we assigned a pathway-level $\mathrm{Z}$ score $\left(Z_{A}\right)$ as a sum of all individual KEGG pathway protein 1348 member $\mathrm{Z}$ scores divided by the square root of number of member proteins $(k)$

$$
Z_{A}=\sum_{i=1}^{k} Z_{i} / \sqrt{k}
$$

1351 Second, each pathway-level $Z$ score $\left(Z_{A}\right)$ was calibrated against null expected mean $\left(\mu_{k}\right)$ and

1352 standard deviation $\left(\sigma_{k}\right)$ for a given pathway consisting of $k$ member proteins, which are 1353 empirically estimated from 10,000 randomly selected gene sets of size $k$ from the BioGRID 1354 protein-protein interaction network.

$$
S_{A}=\left(Z_{A}-\mu_{k}\right) / \sigma_{k}
$$


1357 The analysis results are then organized by KEGG BRITE functional classification scheme. This

1358 allows to compare the relative significance of KEGG pathways (Kanehisa and Goto, 2000;

1359 Kanehisa et al., 2016; Kanehisa et al., 2017) within functionally related BRITE categories as well

1360 as between distinct BRITE categories.

1362 Spatial clustering analysis: We examined spatial clustering between METTL7B true pulldown

1363 proteins and KEGG pathways in protein-protein interaction network and evaluated the significance

1364 of the spatial clustering between them as a function of network distance. To this end, we extended

1365 existing implementation of spatial statics $\mathrm{K}^{\text {net }}$ function (Cornish and Markowetz, 2014) to allow

1366 us to examine spatial correlation of two types of points (e.g., a set of proteins in a KEGG pathway

1367 and a set of METTL7B true pulldown proteins) in the protein-protein interaction network. We

1368 define spatial correlation of two groups (e.g., group $a$ and group $b$ ) of points as follows,

1369

1371

1372

1373

1374

1375

1376 1377 a weight of each protein in two groups $\left(\overline{p_{a}}=\frac{1}{N} \sum_{i}^{N} p_{i}\right.$ and $\left.\overline{p_{b}}=\frac{1}{N} \sum_{j}^{N} p_{j}\right)$. To estimate the statistical 1378 significance of $\mathrm{K}^{\text {net,cross }}(s)$ across network distance, we randomly permuted group membership

Here, $p_{i}$ and $p_{j}$ denote group membership for all the vertex of protein-protein interaction network. (e.g., $\quad p_{i}=1$ and $p_{j}=1$ if a protein is a member of group $a$ and group $b . p_{i}=0$ and $p_{j}=0$ otherwise). The $d(i, j)$ denotes network distance between a pair of proteins in two groups, $a$ and b. $I(d(i, j)<s)$ is an indicator function with value of 1 for a pair of proteins in the two groups closer than network distance s and 0 otherwise. $1 / \overline{p_{a}}$ and $1 / \overline{p_{b}}$ are normalization constants giving 
1379 in the network and obtained the significance as a Z-score: $\mathrm{Z}^{\text {net,cross }}(s)=\frac{\mathrm{K}^{\text {net,cross }}(\mathrm{s})-\overline{\mathrm{K}^{\text {net,cross }}(\mathrm{s})}}{\sigma_{\mathrm{K}} \text { net,cross }}$

1380 where $\overline{\mathrm{K}^{\text {net,cross }}(\mathrm{s})}$ and $\sigma_{\mathrm{K}^{\text {net,cross }}}$ are mean and variance of empirical null expectation obtained

1381 from the random permutations. Thus, $\mathrm{Z}^{\text {net,cross }}(s)$ provides an insight about a profile of spatial

1382 overlap between the two groups of molecular signatures in protein-protein interaction network.

1383

$1384 \quad \underline{\text { ELISA }}$

1385 To determine the differences in A $\beta$ generation, 20000 N2a-APP cells per well were plated in 96-

1386 well plate. After four hours, cells were transfected with pDTET-EGFP or pDTET-METTL7B-

1387 EGFP using PolyJet reagent (SignaGen). Four hours post transfection, cells were rinsed twice with

1388 PBS and incubated in fresh media supplemented with $200 \mathrm{nM}$ doxycycline. After 48 hours,

1389 conditioned media was collected and supplemented with protease inhibitor cocktail (P-2714,

1390 Sigma-Aldrich). Cells were lysed in Cell Extraction Buffer (Thermo Fisher Scientific)

1391 supplemented with protease inhibitor cocktail. Cell culture supernatants were analyzed in 1392 duplicates on $A \beta 40$ and $A \beta 42$ colorimetric ELISAs per manufacturer's protocol (KHB3481 and

1393 KHB3544, Invitrogen). A $\beta$ concentrations were normalized per total cell protein concentrations, 1394 measured by the Rapid Gold BCA Protein Assay (\#A53225, Pierce).

$1397 \quad$ Immunoblotting and silver stain

1398 Tissue sample preparation: Tissue was lysed in PBS with $0.01 \%$ Tween-20 and protease inhibitor

1399 cocktail (P-2714, Sigma-Aldrich), and sonicated in two sessions (30 pulses at an output level of 3

1400 using a Microson Ultrasonic Cell Disruptor [Misonix]) with 1-minute rest on ice between sessions. 
1401 Samples were centrifuged at $14000 \mathrm{~g}$ for 10 minutes at $4{ }^{\circ} \mathrm{C}$. Total protein concentrations were 1402 measured by the Bradford assay (\#23246, Pierce).

Immunoblotting: Samples were mixed with NuPAGE LDS Loading Buffer (NP0007)

1405 supplemented with $50 \mathrm{mM} \mathrm{DTT}$, incubated at $72{ }^{\circ} \mathrm{C}$ for 10 minutes, and loaded on $4-12 \%$ Bis-Tris 1406 gel (NP0321, Thermo Fisher Scientific). Proteins were transferred to a $0.2 \mu \mathrm{m}$ PVDF membrane

1407 (\#162-0218, Bio-Rad), blocked with 5\% non-fat milk or BSA in 1\% TBST buffer, and blotted with 1408 appropriate primary and secondary HRP-conjugated antibodies. The signal was developed with 1409 SuperSignal West Pico Plus Chemiluminescent Substrate (\#34577, Pierce) and visualized on 1410 G:BOX Chemi XRQ (Syngene) system.

1412 Silver stain: 5\% of HaloTag eluates were prepared as above and electrophoresed on 4-12\% Bis1413 Tris gel. Gel was processed using Silver Stain for Mass Spectrometry kit per manufacturer's 1414 instructions (\#24600, Pierce).

\section{SAM assay}

1417 Custom made recombinant METTL7B was expressed in E. Coli ArcticExpress and purified from 1418 inclusion bodies by GenScript. Recombinant RTN3, RTN4, LRP1, and APP peptide were 1419 purchased directly from vendors. SAMfluoro Methyltransferase Assay (786-431, G-Biosciences) 1420 was performed per manufacturer's instructions using $\sim 2 \mu \mathrm{g}$ of METTL7B and $\sim 1 \mu \mathrm{g}$ of substrate 1421 protein. Recombinant proteins were incubated with or without METTL7B in triplicate wells. 1422 Assay was performed at $37^{\circ} \mathrm{C}$ and resorufin fluorescence was measured on GloMax Multi 
1423 Detection System (Promega) plate reader with an excitation wavelength of 530-540 nm and an 1424 emission wavelength of 585-595 $\mathrm{nm}$.

$\underline{\text { RNA isolation and digital droplet PCR }}$

1427 Total RNA was extracted from human and mouse brain tissue samples, or cultured cells, using

1428 RNAeasy Plus Mini Kit (\#74134, Qiagen) per manufacturer's protocol. RNA concentrations and

1429 quality were determined using R6K ScreenTape (\#5067-5576, Agilent) and TapeStation analyzer

1430 (Agilent). cDNA was synthesized from $1 \mu \mathrm{g}$ of total RNA using SuperScript III First-Strand

1431 Synthesis kit (\#18080051, Invitrogen) and random primers. Digital droplet PCR was performed

1432 using QX200 Droplet Digital PCR (Bio-Rad) and data was normalized to TBP expression. PCR

1433 amplification was performed using primer sets and probes listed in Table S4. 


\section{References}

Abul-Husn, N.S., Bushlin, I., Moron, J.A., Jenkins, S.L., Dolios, G., Wang, R., Iyengar, R., Ma'ayan, A., and Devi, L.A. (2009). Systems approach to explore components and interactions in the presynapse. Proteomics 9, 3303-3315.

Aibar, S., Gonzalez-Blas, C.B., Moerman, T., Huynh-Thu, V.A., Imrichova, H., Hulselmans, G., Rambow, F., Marine, J.C., Geurts, P., Aerts, J., et al. (2017). SCENIC: single-cell regulatory network inference and clustering. Nat Methods 14, 1083-1086.

Alzheimer, A., Stelzmann, R.A., Schnitzlein, H.N., and Murtagh, F.R. (1995). An English translation of Alzheimer's 1907 paper, "Uber eine eigenartige Erkankung der Hirnrinde". Clin Anat $8,429-431$.

Andersen, P. (2007). The hippocampus book (Oxford; New York: Oxford University Press).

Bakken, T.E., Miller, J.A., Luo, R., Bernard, A., Bennett, J.L., Lee, C.K., Bertagnolli, D., Parikshak, N.N., Smith, K.A., Sunkin, S.M., et al. (2015). Spatiotemporal dynamics of the postnatal developing primate brain transcriptome. Hum Mol Genet 24, 4327-4339.

Ballatore, C., Lee, V.M., and Trojanowski, J.Q. (2007). Tau-mediated neurodegeneration in Alzheimer's disease and related disorders. Nat Rev Neurosci 8, 663-672.

Berg, D.A., Su, Y., Jimenez-Cyrus, D., Patel, A., Huang, N., Morizet, D., Lee, S., Shah, R., Ringeling, F.R., Jain, R., et al. (2019). A Common Embryonic Origin of Stem Cells Drives Developmental and Adult Neurogenesis. Cell 177, 654-668 e615.

Blumcke, I., Pauli, E., Clusmann, H., Schramm, J., Becker, A., Elger, C., Merschhemke, M., Meencke, H.J., Lehmann, T., von Deimling, A., et al. (2007). A new clinico-pathological classification system for mesial temporal sclerosis. Acta Neuropathol 113, 235-244.

Boldrini, M., Fulmore, C.A., Tartt, A.N., Simeon, L.R., Pavlova, I., Poposka, V., Rosoklija, G.B., Stankov, A., Arango, V., Dwork, A.J., et al. (2018). Human Hippocampal Neurogenesis Persists throughout Aging. Cell Stem Cell 22, 589-599 e585.

Braak, H., and Del Trecidi, K. (2015). Neuroanatomy and pathology of sporadic Alzheimer's disease. Adv Anat Embryol Cell Biol 215, 1-162.

Breunig, J.J., Sarkisian, M.R., Arellano, J.I., Morozov, Y.M., Ayoub, A.E., Sojitra, S., Wang, B., Flavell, R.A., Rakic, P., and Town, T. (2008). Primary cilia regulate hippocampal neurogenesis by mediating sonic hedgehog signaling. Proc Natl Acad Sci U S A 105, 13127-13132.

Brill, L.M., Motamedchaboki, K., Wu, S., and Wolf, D.A. (2009). Comprehensive proteomic analysis of Schizosaccharomyces pombe by two-dimensional HPLC-tandem mass spectrometry. Methods 48, 311-319. 
Butler, A., Hoffman, P., Smibert, P., Papalexi, E., and Satija, R. (2018). Integrating single-cell transcriptomic data across different conditions, technologies, and species. Nat Biotechnol 36, 411 420.

Buzsaki, G., and Moser, E.I. (2013). Memory, navigation and theta rhythm in the hippocampalentorhinal system. Nat Neurosci 16, 130-138.

Caparros-Martin, J.A., Valencia, M., Reytor, E., Pacheco, M., Fernandez, M., Perez-Aytes, A., Gean, E., Lapunzina, P., Peters, H., Goodship, J.A., et al. (2013). The ciliary Evc/Evc2 complex interacts with Smo and controls Hedgehog pathway activity in chondrocytes by regulating Sufu/Gli3 dissociation and Gli3 trafficking in primary cilia. Hum Mol Genet 22, 124-139.

Cardoso-Moreira, M., Halbert, J., Valloton, D., Velten, B., Chen, C., Shao, Y., Liechti, A., Ascencao, K., Rummel, C., Ovchinnikova, S., et al. (2019). Gene expression across mammalian organ development. Nature 571, 505-509.

Cembrowski, M.S., Bachman, J.L., Wang, L., Sugino, K., Shields, B.C., and Spruston, N. (2016a). Spatial Gene-Expression Gradients Underlie Prominent Heterogeneity of CA1 Pyramidal Neurons. Neuron 89, 351-368.

Cembrowski, M.S., Phillips, M.G., DiLisio, S.F., Shields, B.C., Winnubst, J., Chandrashekar, J., Bas, E., and Spruston, N. (2018). Dissociable Structural and Functional Hippocampal Outputs via Distinct Subiculum Cell Classes. Cell 173, 1280-1292 e1218.

Cembrowski, M.S., Wang, L., Sugino, K., Shields, B.C., and Spruston, N. (2016b). Hipposeq: a comprehensive RNA-seq database of gene expression in hippocampal principal neurons. Elife 5, e14997.

Cenquizca, L.A., and Swanson, L.W. (2007). Spatial organization of direct hippocampal field CA1 axonal projections to the rest of the cerebral cortex. Brain Res Rev 56, 1-26.

Choi, H., Larsen, B., Lin, Z.Y., Breitkreutz, A., Mellacheruvu, D., Fermin, D., Qin, Z.S., Tyers, M., Gingras, A.C., and Nesvizhskii, A.I. (2011). SAINT: probabilistic scoring of affinity purification-mass spectrometry data. Nat Methods 8, 70-73.

Choi, S.H., Kim, Y.H., Hebisch, M., Sliwinski, C., Lee, S., D'Avanzo, C., Chen, H., Hooli, B., Asselin, C., Muffat, J., et al. (2014). A three-dimensional human neural cell culture model of Alzheimer's disease. Nature 515, 274-278.

Cipriani, S., Ferrer, I., Aronica, E., Kovacs, G.G., Verney, C., Nardelli, J., Khung, S., Delezoide, A.L., Milenkovic, I., Rasika, S., et al. (2018). Hippocampal Radial Glial Subtypes and Their Neurogenic Potential in Human Fetuses and Healthy and Alzheimer's Disease Adults. Cereb Cortex 28, 2458-2478.

Cipriani, S., Nardelli, J., Verney, C., Delezoide, A.L., Guimiot, F., Gressens, P., and AdleBiassette, H. (2016). Dynamic Expression Patterns of Progenitor and Pyramidal Neuron Layer Markers in the Developing Human Hippocampus. Cereb Cortex 26, 1255-1271. 
Cornish, A.J., and Markowetz, F. (2014). SANTA: quantifying the functional content of molecular networks. PLoS Comput Biol 10, e1003808.

Couillard-Despres, S., Winner, B., Schaubeck, S., Aigner, R., Vroemen, M., Weidner, N., Bogdahn, U., Winkler, J., Kuhn, H.G., and Aigner, L. (2005). Doublecortin expression levels in adult brain reflect neurogenesis. Eur J Neurosci 21, 1-14.

Davies, D.C., Horwood, N., Isaacs, S.L., and Mann, D.M. (1992). The effect of age and Alzheimer's disease on pyramidal neuron density in the individual fields of the hippocampal formation. Acta Neuropathol 83, 510-517.

Demeter, S., Rosene, D.L., and Van Hoesen, G.W. (1990). Fields of origin and pathways of the interhemispheric commissures in the temporal lobe of macaques. J Comp Neurol 302, 29-53.

Dennis, C.V., Suh, L.S., Rodriguez, M.L., Kril, J.J., and Sutherland, G.T. (2016). Human adult neurogenesis across the ages: An immunohistochemical study. Neuropathol Appl Neurobiol 42, 621-638.

Di Paolo, G., and Kim, T.W. (2011). Linking lipids to Alzheimer's disease: cholesterol and beyond. Nat Rev Neurosci 12, 284-296.

Dull, T., Zufferey, R., Kelly, M., Mandel, R.J., Nguyen, M., Trono, D., and Naldini, L. (1998). A third-generation lentivirus vector with a conditional packaging system. J Virol 72, 8463-8471.

Edler, M.K., Sherwood, C.C., Meindl, R.S., Hopkins, W.D., Ely, J.J., Erwin, J.M., Mufson, E.J., Hof, P.R., and Raghanti, M.A. (2017). Aged chimpanzees exhibit pathologic hallmarks of Alzheimer's disease. Neurobiol Aging 59, 107-120.

Eriksson, P.S., Perfilieva, E., Bjork-Eriksson, T., Alborn, A.M., Nordborg, C., Peterson, D.A., and Gage, F.H. (1998). Neurogenesis in the adult human hippocampus. Nat Med 4, 1313-1317.

Finch, C.E., and Austad, S.N. (2015). Commentary: is Alzheimer's disease uniquely human? Neurobiol Aging 36, 553-555.

Fischer, O. (1907). Miliare Nekrosen mit drusigen Wucherungen der Neurofibrillen, eine regelmassige Veranderung der Hirnrinde bei seniler Demenz. Monatsschr Psychiatr Neurol 22, 361-372.

Freund, T.F. (2002). Changes in the views of neuronal connectivity and communication after Cajal: examples from the hippocampus. Prog Brain Res 136, 203-213.

Glenner, G.G., and Wong, C.W. (1984). Alzheimer's disease: initial report of the purification and characterization of a novel cerebrovascular amyloid protein. Biochem Biophys Res Commun 120, 885-890.

Gloor, P. (1997). The temporal lobe and limbic system (New York: Oxford University Press). 
Habib, N., Avraham-Davidi, I., Basu, A., Burks, T., Shekhar, K., Hofree, M., Choudhury, S.R., Aguet, F., Gelfand, E., Ardlie, K., et al. (2017). Massively parallel single-nucleus RNA-seq with DroNc-seq. Nat Methods 14, 955-958.

Haghverdi, L., Lun, A.T.L., Morgan, M.D., and Marioni, J.C. (2018). Batch effects in single-cell RNA-sequencing data are corrected by matching mutual nearest neighbors. Nat Biotechnol 36 , 421-427.

Hamilton, L.K., Dufresne, M., Joppe, S.E., Petryszyn, S., Aumont, A., Calon, F., Barnabe-Heider, F., Furtos, A., Parent, M., Chaurand, P., et al. (2015). Aberrant Lipid Metabolism in the Forebrain Niche Suppresses Adult Neural Stem Cell Proliferation in an Animal Model of Alzheimer's Disease. Cell Stem Cell 17, 397-411.

Hardy, J., and Selkoe, D.J. (2002). The amyloid hypothesis of Alzheimer's disease: progress and problems on the road to therapeutics. Science 297, 353-356.

Hochgerner, H., Zeisel, A., Lonnerberg, P., and Linnarsson, S. (2018). Conserved properties of dentate gyrus neurogenesis across postnatal development revealed by single-cell RNA sequencing. Nat Neurosci 21, 290-299.

Hodge, R.D., Bakken, T.E., Miller, J.A., Smith, K.A., Barkan, E.R., Graybuck, L.T., Close, J.L., Long, B., Johansen, N., Penn, O., et al. (2019). Conserved cell types with divergent features in human versus mouse cortex. Nature 573, 61-68.

Hodges, B.D., and Wu, C.C. (2010). Proteomic insights into an expanded cellular role for cytoplasmic lipid droplets. J Lipid Res 51, 262-273.

Hof, P.R., and Morrison, J.H. (1990). Quantitative analysis of a vulnerable subset of pyramidal neurons in Alzheimer's disease: II. Primary and secondary visual cortex. J Comp Neurol 301, 5564.

Hoogland, P.V., and Vermeulen-Vanderzee, E. (1989). Efferent connections of the dorsal cortex of the lizard Gekko gecko studied with Phaseolus vulgaris-leucoagglutinin. J Comp Neurol 285, 289-303.

Hook, B. (2014). Cleaner Protein with HaloTag Purification Resins (Promega Corporation Web site.: Promega).

Ideker, T., Ozier, O., Schwikowski, B., and Siegel, A.F. (2002). Discovering regulatory and signalling circuits in molecular interaction networks. Bioinformatics 18 Suppl 1, S233-240.

Ishizuka, N. (2001). Laminar organization of the pyramidal cell layer of the subiculum in the rat. J Comp Neurol 435, 89-110.

Jin, K., Peel, A.L., Mao, X.O., Xie, L., Cottrell, B.A., Henshall, D.C., and Greenberg, D.A. (2004). Increased hippocampal neurogenesis in Alzheimer's disease. Proc Natl Acad Sci U S A 101, 343347. 
Kanehisa, M., Furumichi, M., Tanabe, M., Sato, Y., and Morishima, K. (2017). KEGG: new perspectives on genomes, pathways, diseases and drugs. Nucleic Acids Res 45, D353-D361.

Kanehisa, M., and Goto, S. (2000). KEGG: kyoto encyclopedia of genes and genomes. Nucleic Acids Res 28, 27-30.

Kanehisa, M., Sato, Y., Kawashima, M., Furumichi, M., and Tanabe, M. (2016). KEGG as a reference resource for gene and protein annotation. Nucleic Acids Res 44, D457-462.

Kang, H.J., Kawasawa, Y.I., Cheng, F., Zhu, Y., Xu, X., Li, M., Sousa, A.M., Pletikos, M., Meyer, K.A., Sedmak, G., et al. (2011). Spatio-temporal transcriptome of the human brain. Nature 478, 483-489.

Karran, E., and De Strooper, B. (2016). The amyloid cascade hypothesis: are we poised for success or failure? J Neurochem 139 Suppl 2, 237-252.

Kempermann, G., Gage, F.H., Aigner, L., Song, H., Curtis, M.A., Thuret, S., Kuhn, H.G., Jessberger, S., Frankland, P.W., Cameron, H.A., et al. (2018). Human Adult Neurogenesis: Evidence and Remaining Questions. Cell Stem Cell 23, 25-30.

Kim, D.I., Kc, B., Zhu, W., Motamedchaboki, K., Doye, V., and Roux, K.J. (2014). Probing nuclear pore complex architecture with proximity-dependent biotinylation. Proceedings of the National Academy of Sciences 111, E2453 LP-E2461.

Klausberger, T., and Somogyi, P. (2008). Neuronal diversity and temporal dynamics: the unity of hippocampal circuit operations. Science 321, 53-57.

Kriegstein, A.R., and Connors, B.W. (1986). Cellular physiology of the turtle visual cortex: synaptic properties and intrinsic circuitry. J Neurosci 6, 178-191.

Krishnaswami, S.R., Grindberg, R.V., Novotny, M., Venepally, P., Lacar, B., Bhutani, K., Linker, S.B., Pham, S., Erwin, J.A., Miller, J.A., et al. (2016). Using single nuclei for RNA-seq to capture the transcriptome of postmortem neurons. Nat Protoc 11, 499-524.

Lake, B.B., Ai, R., Kaeser, G.E., Salathia, N.S., Yung, Y.C., Liu, R., Wildberg, A., Gao, D., Fung, H.L., Chen, S., et al. (2016). Neuronal subtypes and diversity revealed by single-nucleus RNA sequencing of the human brain. Science 352, 1586-1590.

Lake, B.B., Chen, S., Sos, B.C., Fan, J., Kaeser, G.E., Yung, Y.C., Duong, T.E., Gao, D., Chun, J., Kharchenko, P.V., et al. (2018). Integrative single-cell analysis of transcriptional and epigenetic states in the human adult brain. Nat Biotechnol 36, 70-80.

Lanjakornsiripan, D., Pior, B.J., Kawaguchi, D., Furutachi, S., Tahara, T., Katsuyama, Y., Suzuki, Y., Fukazawa, Y., and Gotoh, Y. (2018). Layer-specific morphological and molecular differences in neocortical astrocytes and their dependence on neuronal layers. Nat Commun 9, 1623. 
Li, M., Santpere, G., Imamura Kawasawa, Y., Evgrafov, O.V., Gulden, F.O., Pochareddy, S., Sunkin, S.M., Li, Z., Shin, Y., Zhu, Y., et al. (2018). Integrative functional genomic analysis of human brain development and neuropsychiatric risks. Science 362 .

Lois, C., Hong, E.J., Pease, S., Brown, E.J., and Baltimore, D. (2002). Germline transmission and tissue-specific expression of transgenes delivered by lentiviral vectors. Science 295, 868-872.

Luzzati, F. (2015). A hypothesis for the evolution of the upper layers of the neocortex through cooption of the olfactory cortex developmental program. Front Neurosci 9, 162.

Mathys, H., Davila-Velderrain, J., Peng, Z., Gao, F., Mohammadi, S., Young, J.Z., Menon, M., He, L., Abdurrob, F., Jiang, X., et al. (2019). Single-cell transcriptomic analysis of Alzheimer's disease. Nature 570, 332-337.

Matsuda, T., and Cepko, C.L. (2004). Electroporation and RNA interference in the rodent retina in vivo and in vitro. Proc Natl Acad Sci U S A 101, 16-22.

McMenemey, W.H. (1940). Alzheimer's Disease: A Report of Six Cases. J Neurol Psychiatry 3, 211-240.

Mercer, A., and Thomson, A.M. (2017). Cornu Ammonis Regions-Antecedents of Cortical Layers? Front Neuroanat 11,83.

Moreno-Jimenez, E.P., Flor-Garcia, M., Terreros-Roncal, J., Rabano, A., Cafini, F., PallasBazarra, N., Avila, J., and Llorens-Martin, M. (2019). Adult hippocampal neurogenesis is abundant in neurologically healthy subjects and drops sharply in patients with Alzheimer's disease. Nat Med 25, 554-560.

Morrison, J.H., and Hof, P.R. (1997). Life and death of neurons in the aging brain. Science 278, 412-419.

Nielsen, J.V., Blom, J.B., Noraberg, J., and Jensen, N.A. (2010). Zbtb20-induced CA1 pyramidal neuron development and area enlargement in the cerebral midline cortex of mice. Cereb Cortex 20, 1904-1914.

Ohara, S., Onodera, M., Simonsen, O.W., Yoshino, R., Hioki, H., Iijima, T., Tsutsui, K.I., and Witter, M.P. (2018). Intrinsic Projections of Layer Vb Neurons to Layers Va, III, and II in the Lateral and Medial Entorhinal Cortex of the Rat. Cell Rep 24, 107-116.

Ohm, T.G. (2007). The dentate gyrus in Alzheimer's disease. Prog Brain Res 163, 723-740.

Park, S.K., Venable, J.D., Xu, T., and Yates, J.R., 3rd (2008). A quantitative analysis software tool for mass spectrometry-based proteomics. Nat Methods 5, 319-322.

Park, S.M., Jang, H.J., and Lee, J.H. (2019). Roles of Primary Cilia in the Developing Brain. Front Cell Neurosci 13, 218. 
1639 Paspalas, C.D., Carlyle, B.C., Leslie, S., Preuss, T.M., Crimins, J.L., Huttner, A.J., van Dyck, C.H., Rosene, D.L., Nairn, A.C., and Arnsten, A.F.T. (2018). The aged rhesus macaque manifests Braak stage III/IV Alzheimer's-like pathology. Alzheimers Dement 14, 680-691.

1642

1643

1644

1645

1646

1647

1648

1649

1650

1651

1652

1653

1654

1655

1656

1657

1658

1659

1660

1661

1662

1663

1664

1665

1666

1667

1668

1669

1670

1671

1672

1673

Patzke, N., Spocter, M.A., Karlsson, K.A.E., Bertelsen, M.F., Haagensen, M., Chawana, R., Streicher, S., Kaswera, C., Gilissen, E., Alagaili, A.N., et al. (2015). In contrast to many other mammals, cetaceans have relatively small hippocampi that appear to lack adult neurogenesis. Brain Struct Funct 220, 361-383.

Perez, S.E., Raghanti, M.A., Hof, P.R., Kramer, L., Ikonomovic, M.D., Lacor, P.N., Erwin, J.M., Sherwood, C.C., and Mufson, E.J. (2013). Alzheimer's disease pathology in the neocortex and hippocampus of the western lowland gorilla (Gorilla gorilla gorilla). J Comp Neurol 521, 43184338.

Qin, W., Ho, L., Pompl, P.N., Peng, Y., Zhao, Z., Xiang, Z., Robakis, N.K., Shioi, J., Suh, J., and Pasinetti, G.M. (2003). Cyclooxygenase (COX)-2 and COX-1 potentiate beta-amyloid peptide generation through mechanisms that involve gamma-secretase activity. J Biol Chem 278, 5097050977.

Rakic, P. (2002). Neurogenesis in adult primates. Prog Brain Res 138, 3-14.

Ramsden, H.L., Surmeli, G., McDonagh, S.G., and Nolan, M.F. (2015). Laminar and dorsoventral molecular organization of the medial entorhinal cortex revealed by large-scale anatomical analysis of gene expression. PLoS Comput Biol 11, e1004032.

Rapoport, S.I. (1989). Hypothesis: Alzheimer's disease is a phylogenetic disease. Med Hypotheses $29,147-150$.

Reiner, A. (1991). A comparison of neurotransmitter-specific and neuropeptide-specific neuronal cell types present in the dorsal cortex in turtles with those present in the isocortex in mammals: implications for the evolution of isocortex. Brain Behav Evol 38, 53-91.

Rhee, S., Kirschen, G.W., Gu, Y., and Ge, S. (2016). Depletion of primary cilia from mature dentate granule cells impairs hippocampus-dependent contextual memory. Sci Rep 6, 34370.

Robinson, P.N., Wollstein, A., Bohme, U., and Beattie, B. (2004). Ontologizing gene-expression microarray data: characterizing clusters with Gene Ontology. Bioinformatics 20, 979-981.

Roux, K.J., Kim, D.I., and Burke, B. (2013). BioID: a screen for protein-protein interactions. Curr Protoc Protein Sci 74, 1923 11-19 2314.

Roux, K.J., Kim, D.I., Raida, M., and Burke, B. (2012). A promiscuous biotin ligase fusion protein identifies proximal and interacting proteins in mammalian cells. J Cell Biol 196, 801-810.

Schirmer, L., Velmeshev, D., Holmqvist, S., Kaufmann, M., Werneburg, S., Jung, D., Vistnes, S., Stockley, J.H., Young, A., Steindel, M., et al. (2019). Neuronal vulnerability and multilineage diversity in multiple sclerosis. Nature 573, 75-82. 
Schmidt-Kastner, R., and Freund, T.F. (1991). Selective vulnerability of the hippocampus in brain ischemia. Neuroscience 40, 599-636.

Schonheit, B., Zarski, R., and Ohm, T.G. (2004). Spatial and temporal relationships between plaques and tangles in Alzheimer-pathology. Neurobiol Aging 25, 697-711.

Serrano-Pozo, A., Frosch, M.P., Masliah, E., and Hyman, B.T. (2011). Neuropathological alterations in Alzheimer disease. Cold Spring Harb Perspect Med 1, a006189.

Shepherd, G.M., and Rowe, T.B. (2017). Neocortical Lamination: Insights from Neuron Types and Evolutionary Precursors. Front Neuroanat 11, 100.

Slomianka, L., Amrein, I., Knuesel, I., Sorensen, J.C., and Wolfer, D.P. (2011). Hippocampal pyramidal cells: the reemergence of cortical lamination. Brain Struct Funct 216, 301-317.

Smyth, G.K. (2005). Limma: linear models for microarray data BT - Bioinformatics and Computational Biology Solutions Using R and Bioconductor. Bioinformatics and Computational Biology Solutions Using R and Bioconductor, 397-420.

Sorrells, S.F., Paredes, M.F., Cebrian-Silla, A., Sandoval, K., Qi, D., Kelley, K.W., James, D., Mayer, S., Chang, J., Auguste, K.I., et al. (2018). Human hippocampal neurogenesis drops sharply in children to undetectable levels in adults. Nature 555, 377-381.

Sorrells, S.F., Paredes, M.F., Velmeshev, D., Herranz-Perez, V., Sandoval, K., Mayer, S., Chang, E.F., Insausti, R., Kriegstein, A.R., Rubenstein, J.L., et al. (2019). Immature excitatory neurons develop during adolescence in the human amygdala. Nat Commun 10, 2748.

Sousa, A.M.M., Zhu, Y., Raghanti, M.A., Kitchen, R.R., Onorati, M., Tebbenkamp, A.T.N., Stutz, B., Meyer, K.A., Li, M., Kawasawa, Y.I., et al. (2017). Molecular and cellular reorganization of neural circuits in the human lineage. Science 358, 1027-1032.

Spalding, K.L., Bergmann, O., Alkass, K., Bernard, S., Salehpour, M., Huttner, H.B., Bostrom, E., Westerlund, I., Vial, C., Buchholz, B.A., et al. (2013). Dynamics of hippocampal neurogenesis in adult humans. Cell 153, 1219-1227.

Stark, C., Breitkreutz, B.J., Reguly, T., Boucher, L., Breitkreutz, A., and Tyers, M. (2006). BioGRID: a general repository for interaction datasets. Nucleic Acids Res 34, D535-539.

Stephan, H. (1975). Allocortex (Springer).

Strittmatter, W.J., Saunders, A.M., Schmechel, D., Pericak-Vance, M., Enghild, J., Salvesen, G.S., and Roses, A.D. (1993). Apolipoprotein E: high-avidity binding to beta-amyloid and increased frequency of type 4 allele in late-onset familial Alzheimer disease. Proc Natl Acad Sci U S A 90, 1977-1981.

Suzuki, W.A., and Amaral, D.G. (2004). Functional neuroanatomy of the medial temporal lobe memory system. Cortex 40, 220-222. 
Tabb, D.L., McDonald, W.H., and Yates, J.R., 3rd (2002). DTASelect and Contrast: tools for assembling and comparing protein identifications from shotgun proteomics. J Proteome Res 1, 2126.

Tang, Q., Ebbesen, C.L., Sanguinetti-Scheck, J.I., Preston-Ferrer, P., Gundlfinger, A., Winterer, J., Beed, P., Ray, S., Naumann, R., Schmitz, D., et al. (2015). Anatomical Organization and Spatiotemporal Firing Patterns of Layer 3 Neurons in the Rat Medial Entorhinal Cortex. J Neurosci $35,12346-12354$.

Tanzi, R.E., and Bertram, L. (2005). Twenty years of the Alzheimer's disease amyloid hypothesis: a genetic perspective. Cell 120, 545-555.

Tasic, B., Yao, Z., Graybuck, L.T., Smith, K.A., Nguyen, T.N., Bertagnolli, D., Goldy, J., Garren, E., Economo, M.N., Viswanathan, S., et al. (2018). Shared and distinct transcriptomic cell types across neocortical areas. Nature 563, 72-78.

Teo, G., Liu, G., Zhang, J., Nesvizhskii, A.I., Gingras, A.C., and Choi, H. (2014). SAINTexpress: improvements and additional features in Significance Analysis of INTeractome software. J Proteomics 100, 37-43.

Thomas, A., Klein, M.S., Stevens, A.P., Reinders, Y., Hellerbrand, C., Dettmer, K., Gronwald, W., Oefner, P.J., and Reinders, J. (2013). Changes in the hepatic mitochondrial and membrane proteome in mice fed a non-alcoholic steatohepatitis inducing diet. J Proteomics 80, 107-122.

Turro, S., Ingelmo-Torres, M., Estanyol, J.M., Tebar, F., Fernandez, M.A., Albor, C.V., Gaus, K., Grewal, T., Enrich, C., and Pol, A. (2006). Identification and characterization of associated with lipid droplet protein 1: A novel membrane-associated protein that resides on hepatic lipid droplets. Traffic 7, 1254-1269.

Uhlen, M., Fagerberg, L., Hallstrom, B.M., Lindskog, C., Oksvold, P., Mardinoglu, A., Sivertsson, A., Kampf, C., Sjostedt, E., Asplund, A., et al. (2015). Proteomics. Tissue-based map of the human proteome. Science 347, 1260419.

Vanlandewijck, M., He, L., Mae, M.A., Andrae, J., Ando, K., Del Gaudio, F., Nahar, K., Lebouvier, T., Lavina, B., Gouveia, L., et al. (2018). A molecular atlas of cell types and zonation in the brain vasculature. Nature 554, 475-480.

Velmeshev, D., Schirmer, L., Jung, D., Haeussler, M., Perez, Y., Mayer, S., Bhaduri, A., Goyal, N., Rowitch, D.H., and Kriegstein, A.R. (2019). Single-cell genomics identifies cell type-specific molecular changes in autism. Science $364,685-689$.

West, M.J., Kawas, C.H., Stewart, W.F., Rudow, G.L., and Troncoso, J.C. (2004). Hippocampal neurons in pre-clinical Alzheimer's disease. Neurobiol Aging 25, 1205-1212.

Wilcock, G.K., and Esiri, M.M. (1982). Plaques, tangles and dementia. A quantitative study. J Neurol Sci 56, 343-356. 
1743 Witter, M.P., Doan, T.P., Jacobsen, B., Nilssen, E.S., and Ohara, S. (2017). Architecture of the 1744 Entorhinal Cortex A Review of Entorhinal Anatomy in Rodents with Some Comparative Notes. 1745 Front Syst Neurosci 11, 46.

1746 Xu, T., Venable, J.D., Park, S.K., Cociorva, D., Lu, B., Liao, L., Wohlschlegel, J., Hewel, J., and 1747 Yates, J.R. (2006). ProLuCID, a fast and sensitive tandem mass spectra-based protein 1748 identification program. Molecular \& cellular proteomics 5.

1749 Zeisel, A., Munoz-Manchado, A.B., Codeluppi, S., Lonnerberg, P., La Manno, G., Jureus, A., 1750 Marques, S., Munguba, H., He, L., Betsholtz, C., et al. (2015). Brain structure. Cell types in the 1751 mouse cortex and hippocampus revealed by single-cell RNA-seq. Science 347, 1138-1142.

1752 Zeldich, E., Chen, C.D., Colvin, T.A., Bove-Fenderson, E.A., Liang, J., Tucker Zhou, T.B., Harris, 1753 D.A., and Abraham, C.R. (2014). The neuroprotective effect of Klotho is mediated via regulation 1754 of members of the redox system. J Biol Chem 289, 24700-24715.

1755 Zhu, Y., Sousa, A.M.M., Gao, T., Skarica, M., Li, M., Santpere, G., Esteller-Cucala, P., Juan, D., 1756 Ferrandez-Peral, L., Gulden, F.O., et al. (2018). Spatiotemporal transcriptomic divergence across 1757 human and macaque brain development. Science 362. 